\title{
Unravelling Protein-DNA Interactions at Molecular Level: A DFT and NCI Study
}

J. González, ${ }^{1}$ I. Baños, ${ }^{2}$ I. León, ${ }^{1}$ J. Contreras-García, ${ }^{3,4}$ E.J. Cocinero, ${ }^{1}$ A. Lesarri, ${ }^{5}$ J. A. Fernández,,1 and J. Millán ${ }^{*}, 2$

${ }^{1}$ Departamento de Química Física, Facultad de Ciencia y Tecnología, Universidad del País Vasco-UPV/EHU, Barrio Sarriena s/n, Leioa, 48940 Spain.

2 Departamento de Química, Facultad de Ciencias, Estudios Agroalimentarios e Informática, Universidad de La Rioja, Madre de Dios, 53, Logroño, 26006 Spain.

3 Sorbonne Universités, UPMC Univ. Paris 06, UMR7616, Laboratoire de Chimie Théorique, F-75005, Paris, France.

${ }^{4}$ CNRS, UMR 7616, Laboratoire de Chimie Théorique, F-75005, Paris, France.

5 Departamento de Química Física y Química Inorgánica, Facultad de Ciencias, Universidad de Valladolid, 47011 Valladolid, Spain.

Contact Address:

Dr. Judith Millán

Dpto. Química

Facultad de Ciencias, Estudios Agroalimentarios e Informática

Universidad de La Rioja,

C/Madre de Dios,53, Logroño 26006, Spain.

Fax: +34941299611;

Tel: +34941299640

E-mail: judith.millan@unirioja.es 
Figure S01. The first 20 calculated structures for aAmC at M06-2X/6-311++G(d,p) level together with their relative stability $\left(\mathrm{kJ} \mathrm{mol}^{-1}\right)$ in brackets. ZPE correction was applied to the energy values.
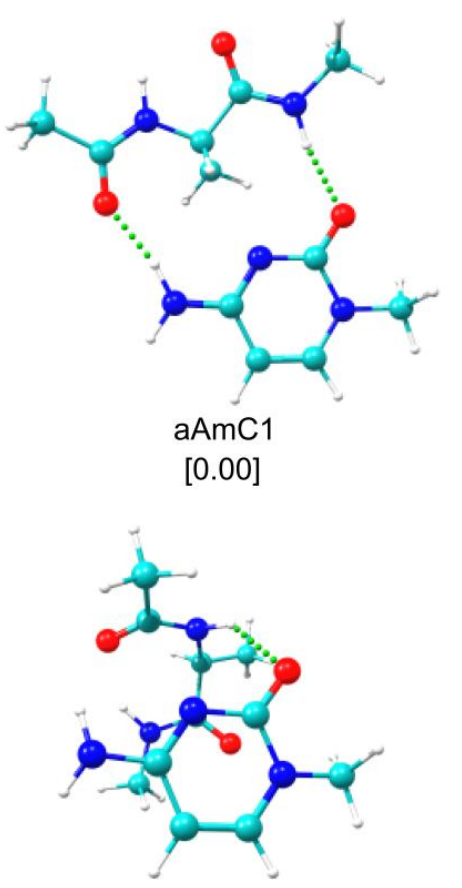

aAmC4

[10.58]

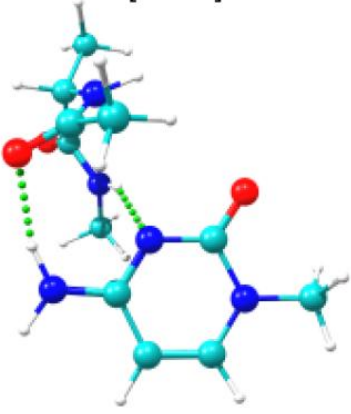

aAmC7

[15.75]

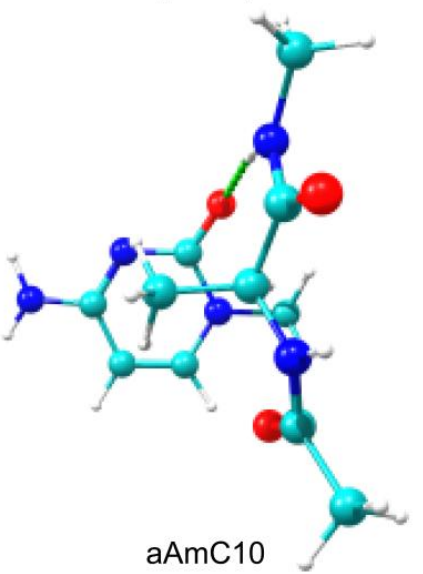

[19.09]

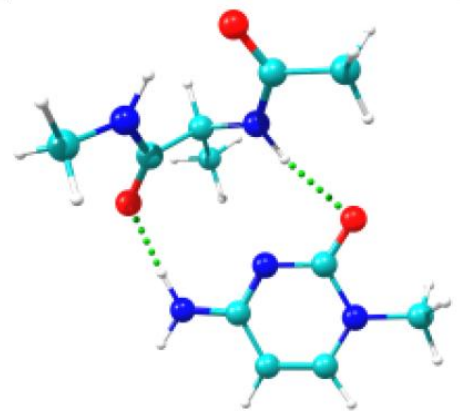

aAmC2

[1.36]

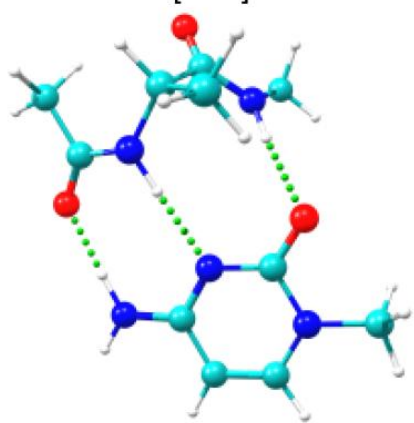

aAmC5

[11.91]

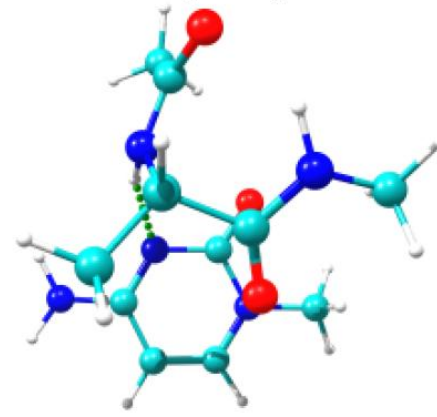

aAmC8

[16.18]

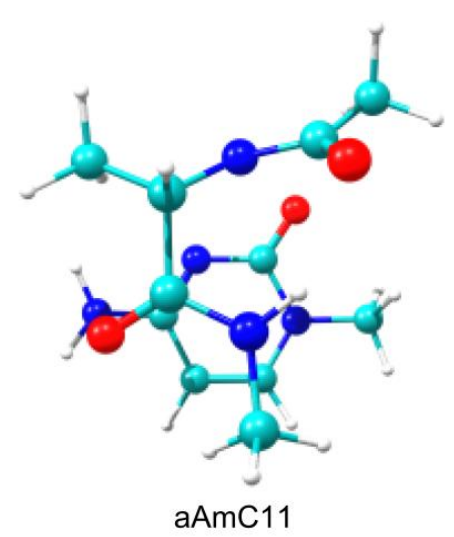

[20.18]

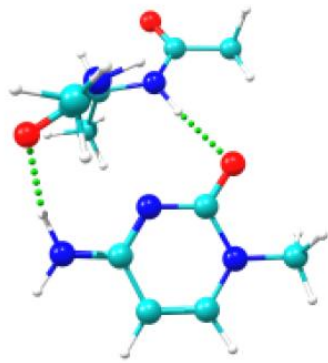

aAmC3

[7.46]

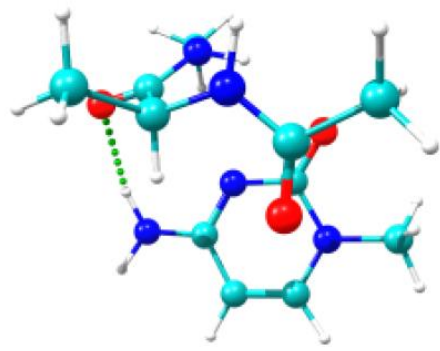

aAmC6

[14.59]
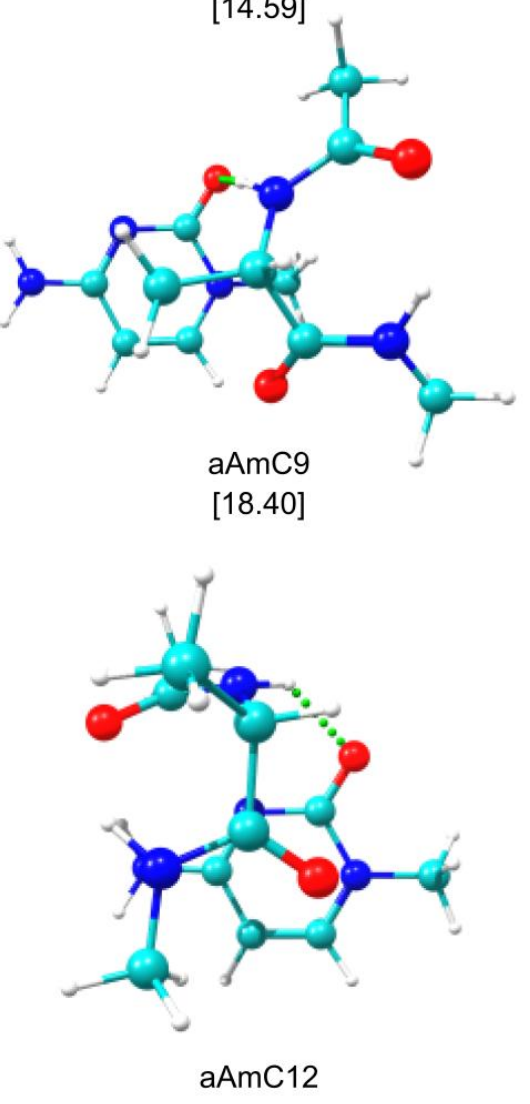

[20.47] 


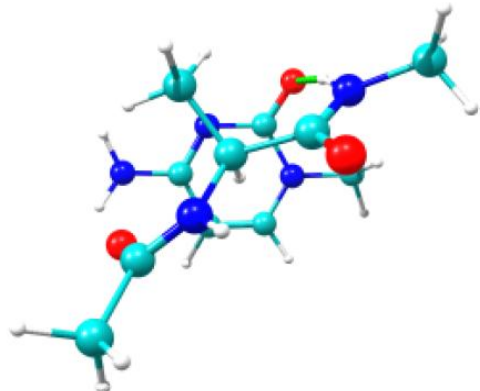

aAmC13

[21.48]

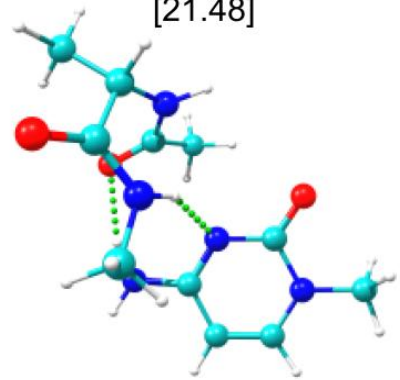

aAmC16

[22.80]

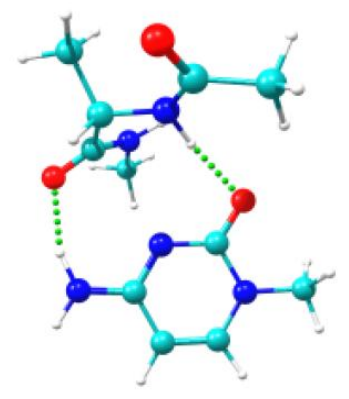

aAmC19

[24.18]

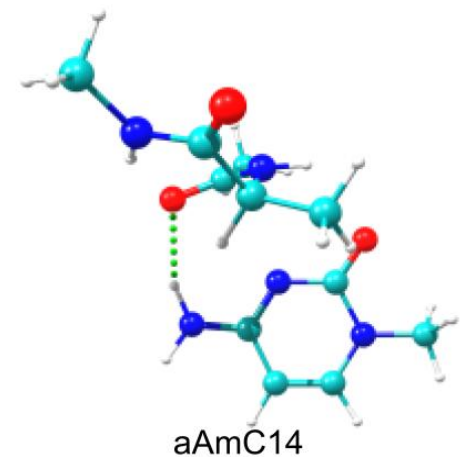

[22.24]

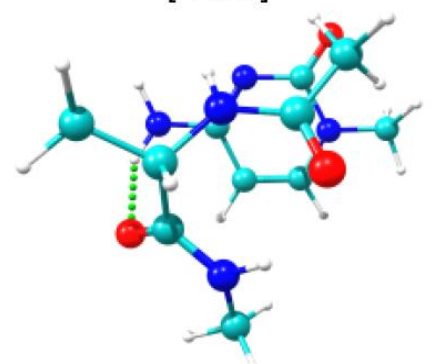

aAmC17

[23.07]

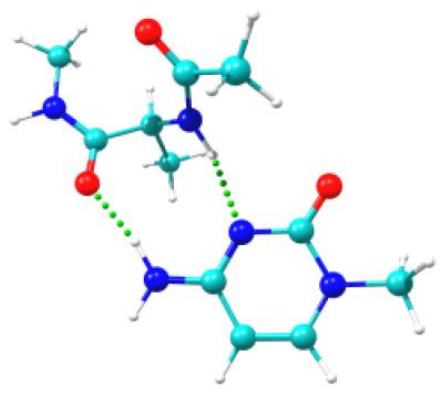

aAmC20

[26.22]

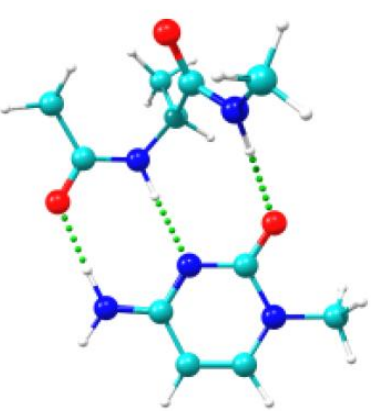

aAmC15

[22.55]

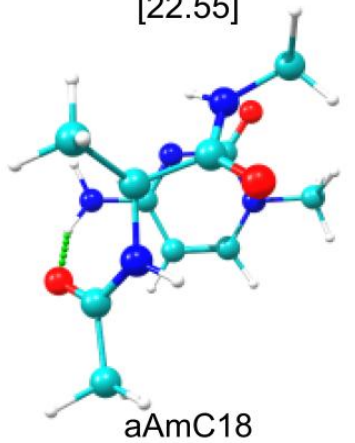

[23.17] 
Figure S02. The first 20 calculated structures for $\mathrm{aVmC}$ at $\mathrm{M} 06-2 \mathrm{X} / 6-311++\mathrm{G}(\mathrm{d}, \mathrm{p})$ level together with their relative stability $\left(\mathrm{kJ} \mathrm{mol}^{-1}\right)$ in brackets. ZPE correction was applied to the energy values.

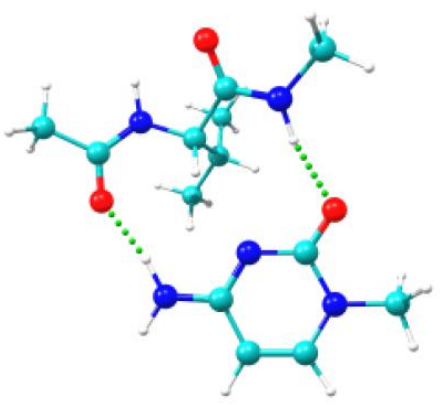

$\mathrm{aVmC1}$

[0.00]

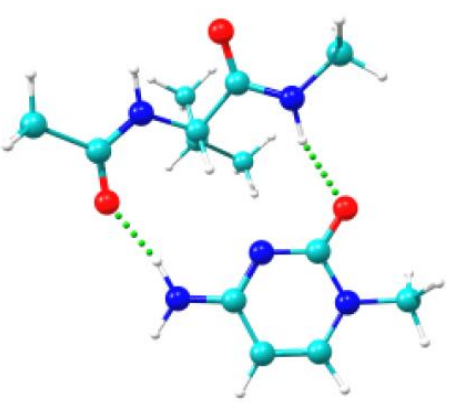

$\mathrm{aVmC4}$

[6.88]

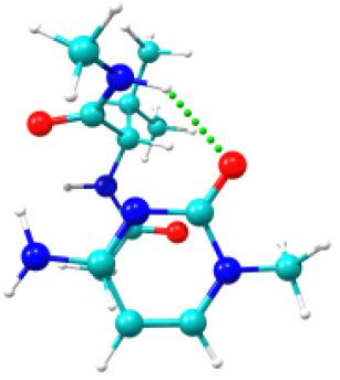

$\mathrm{aVmC7}$

[13.96]

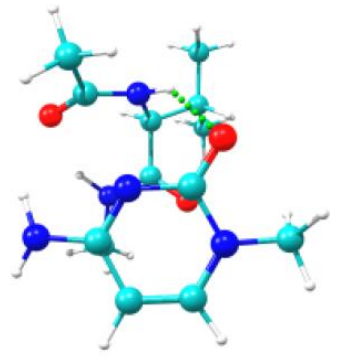

$\mathrm{aVmC10}$

[16.38]

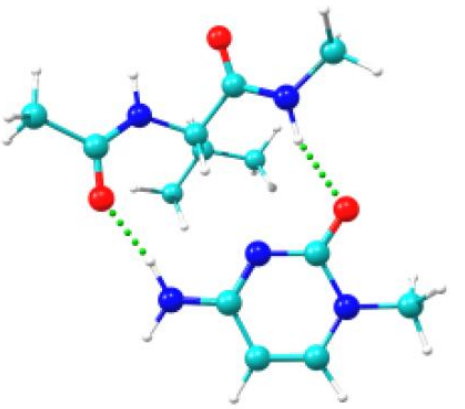

$\mathrm{aVmC2}$

[5.72]

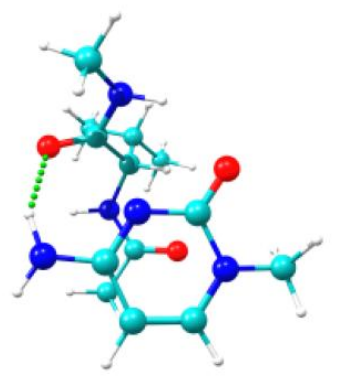

$\mathrm{aVmC5}$

[10.46]

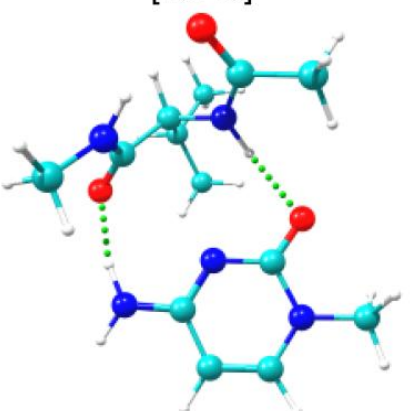

aVmC8

[14.30]

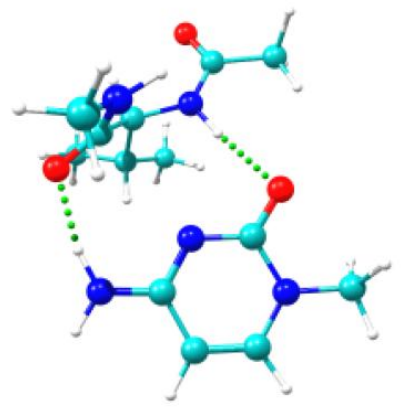

$\mathrm{aVmC} 11$

[18.03]

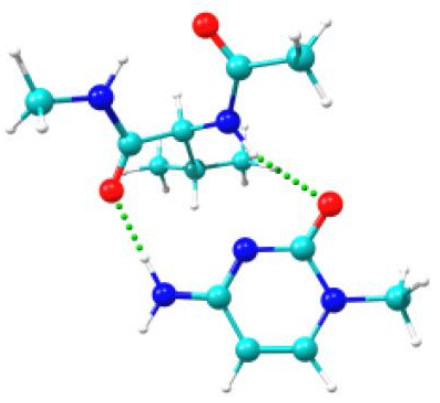

$\mathrm{aVmC3}$

[6.33]

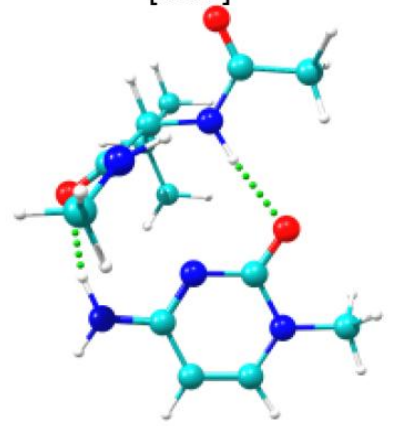

$\mathrm{aVmC6}$

[13.86]

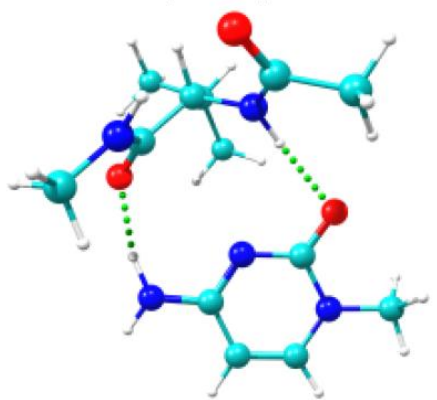

$\mathrm{aVmC9}$

[16.38]

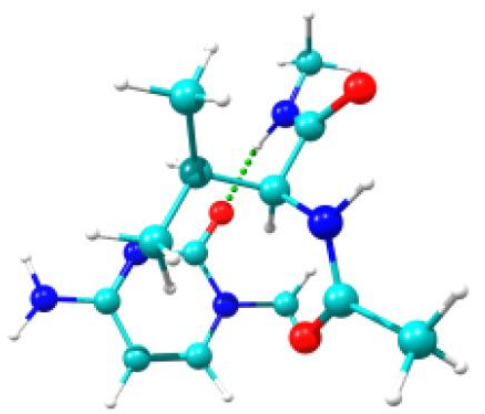

$\mathrm{aVmC} 12$

[18.80] 


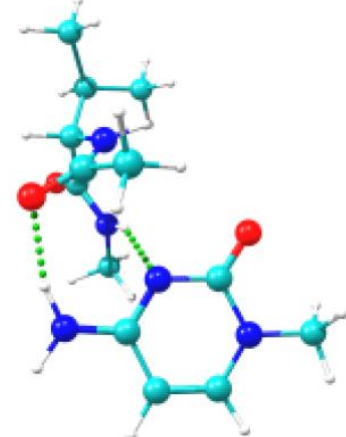

$\mathrm{aVmC} 13$

[21.53]

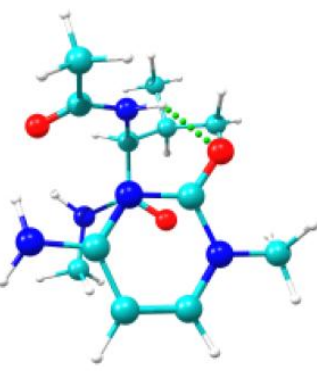

$\mathrm{aVmC16}$

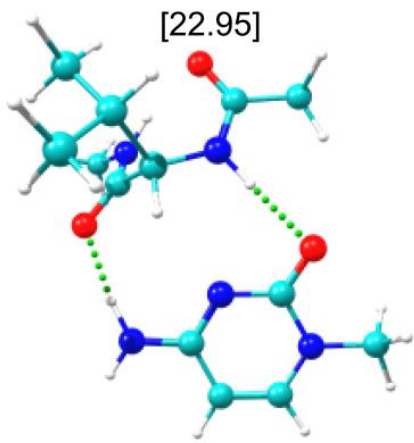

$\mathrm{aVmC19}$

[25.58]

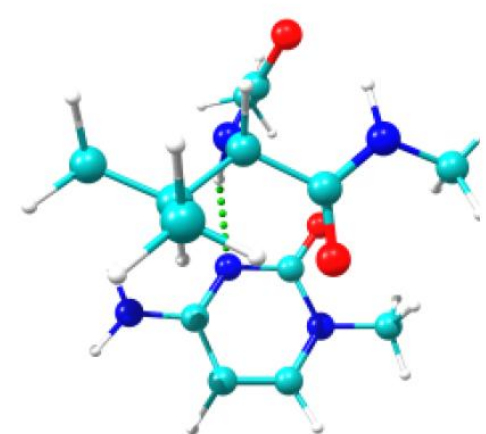

aVmC14

[22.26]

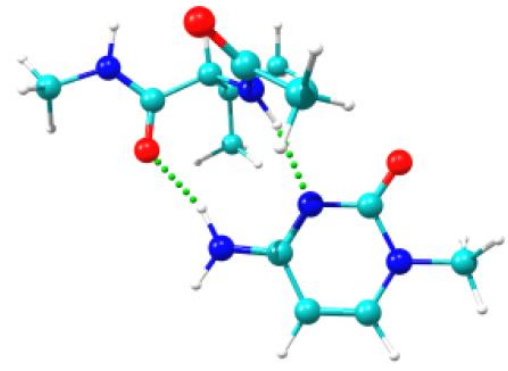

$\mathrm{aVmC} 17$

[23.70]

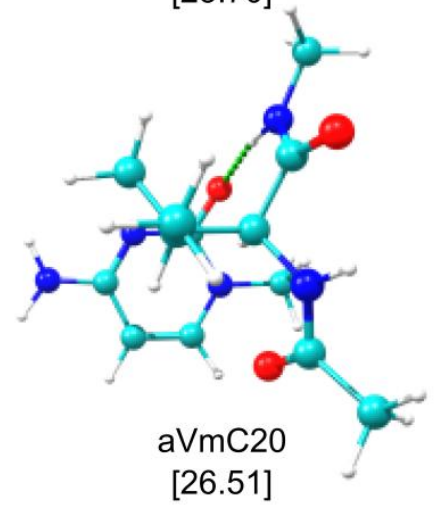

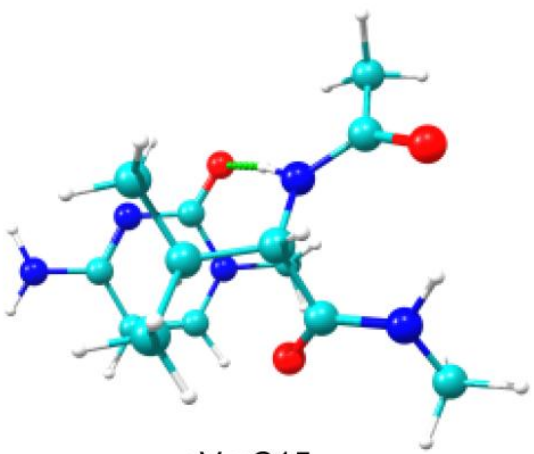

aVmC15

[22.33]

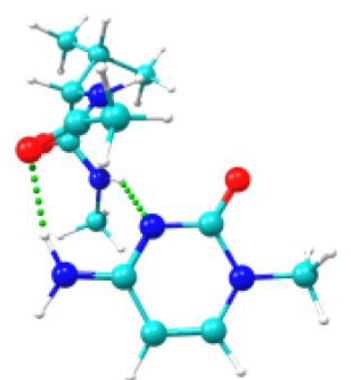

$\mathrm{aVmC} 18$

[23.87] 
Figure S03. The first 20 calculated structures for aImC at M06-2X/6-311++G(d,p) level together with their relative stability $\left(\mathrm{kJ} \mathrm{mol}^{-1}\right)$ in brackets. ZPE correction was applied to the energy values.

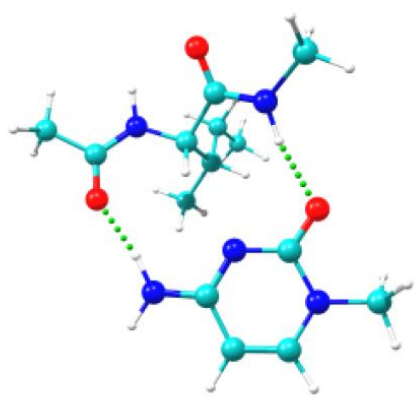

almC1

[0.00]

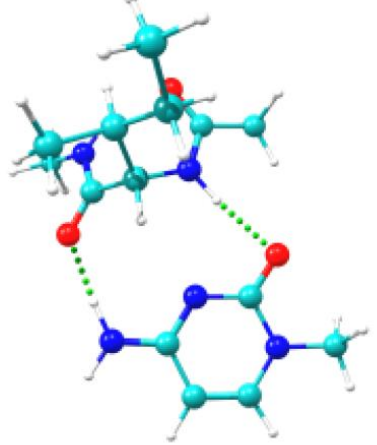

almC4

[8.89]

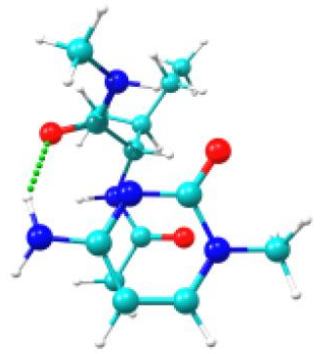

almC7

[13.60]

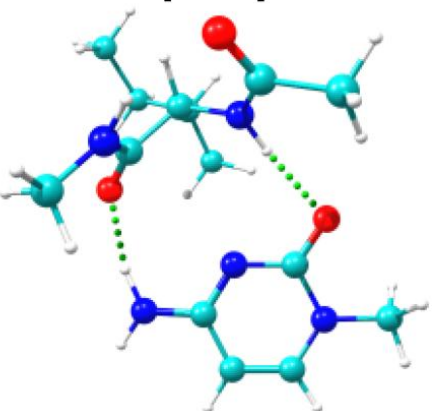

almC10

[16.23]

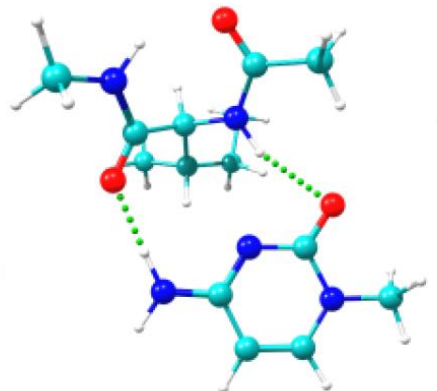

almC2

[3.08]

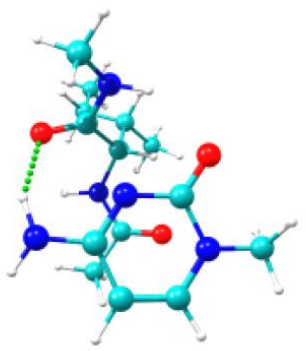

almC5

[10.92]

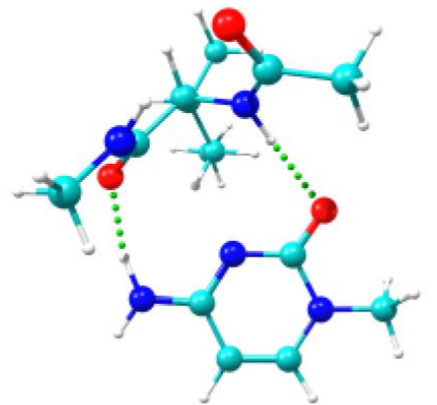

almC8

[13.63]

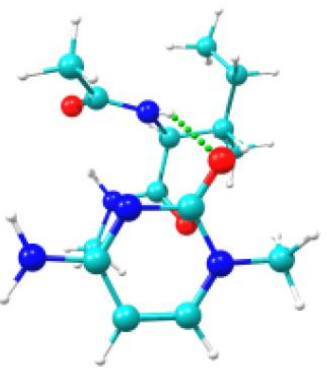

almC11

[17.21]

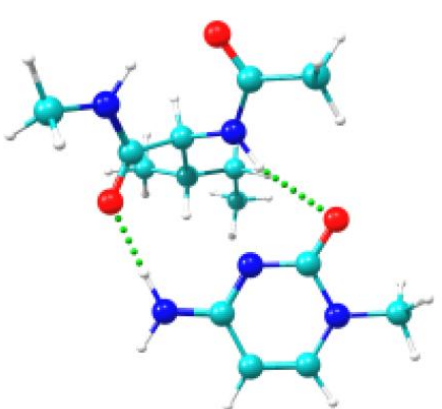

almC3

[4.67]

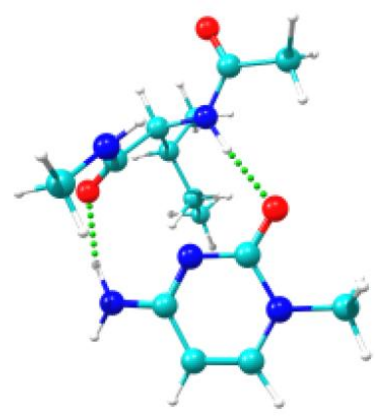

almC6

[12.86]
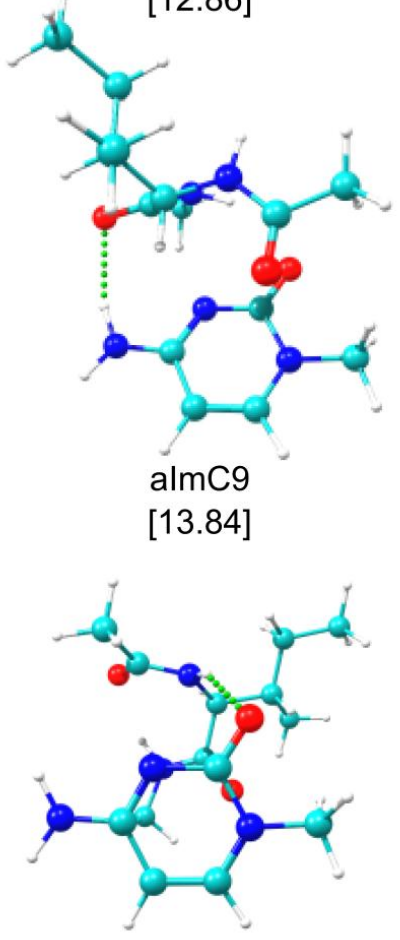

almC12

[17.38] 


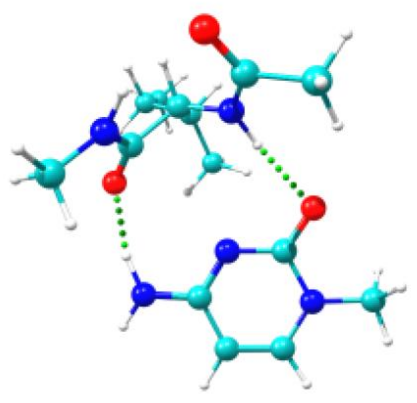

almC13

[17.60]

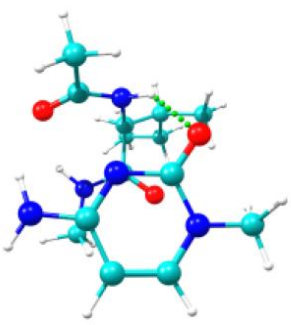

almC16

[19.66]

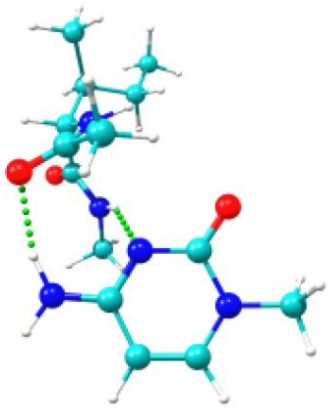

almC19

[20.43]

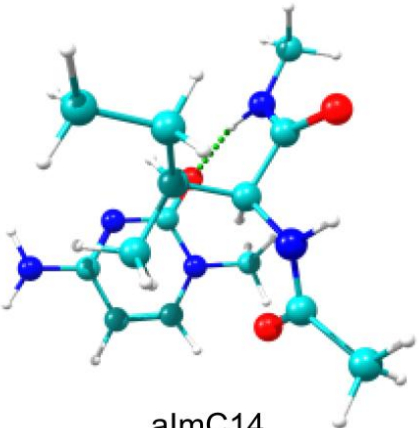

almC14

[18.97]

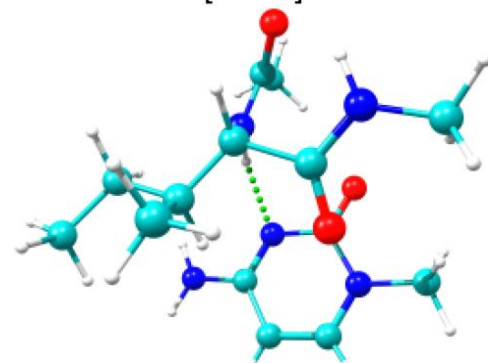

almC17

[19.70]

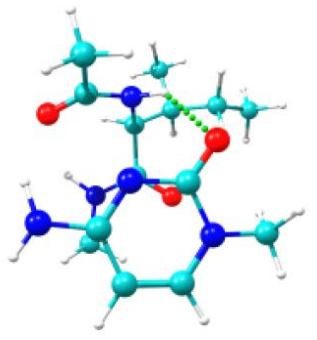

almC20

[21.06]

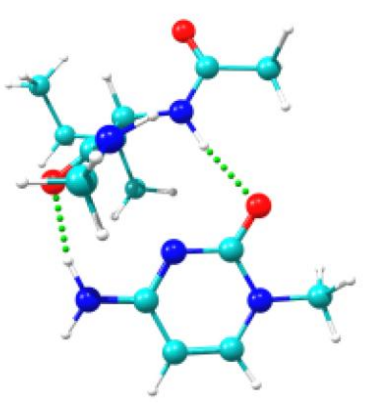

almC15

[19.34]

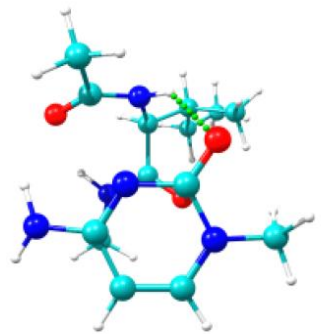

almC18

[20.38] 
Figure S04. The first 20 calculated structures for aNmC at M06-2X/6-311++G(d,p) level together with their relative stability $\left(\mathrm{kJ} \mathrm{mol}^{-1}\right)$ in brackets. ZPE correction was applied to the energy values.

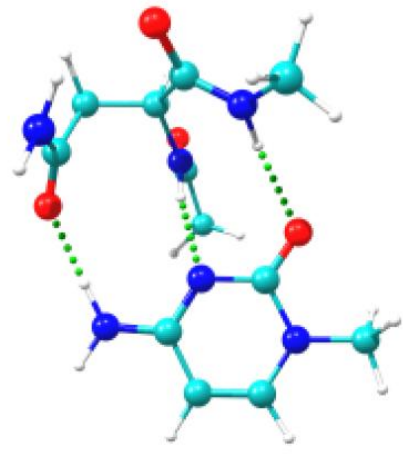

aNmC1

[0.00]

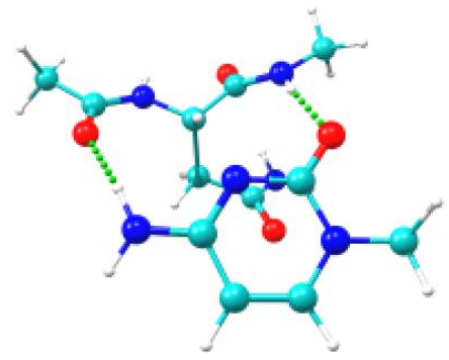

aNmC4

[10.15]

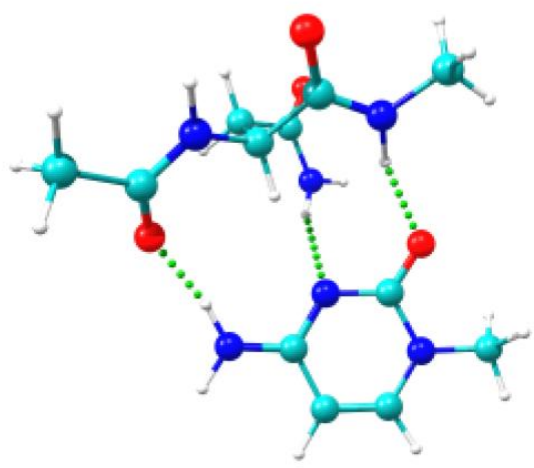

aNmC7

[12.08]

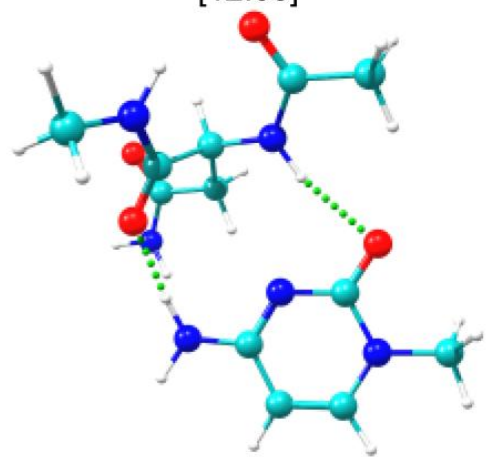

aNmC10

[13.76]

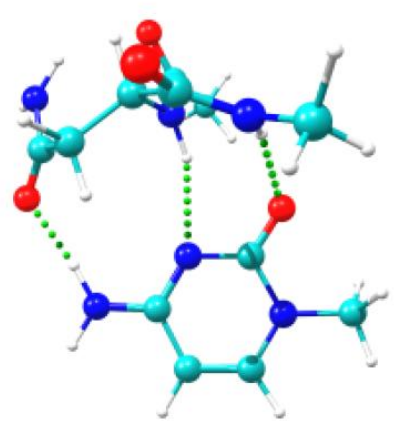

aNmC2

[5.64]

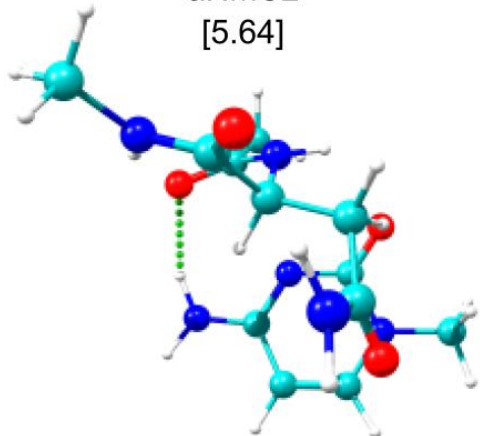

aNmC5

[11.14]

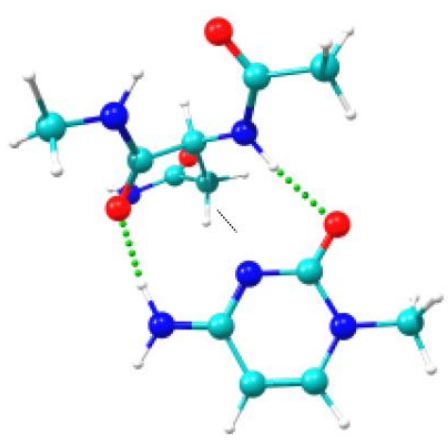

aNmC8

[12.31]

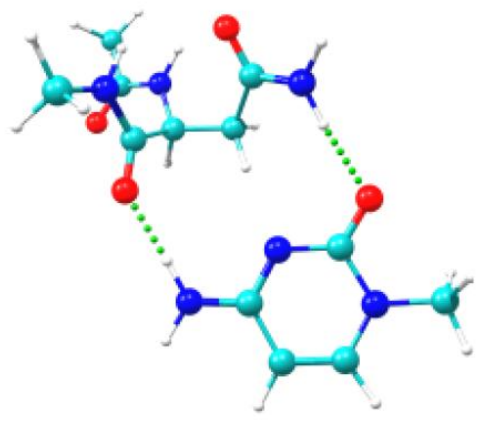

aNmC11

[14.04]

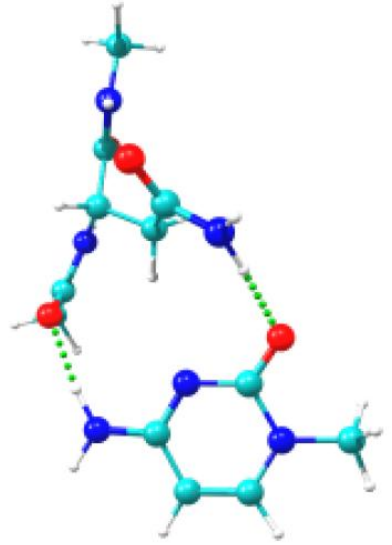

aNmC3

[8.25]

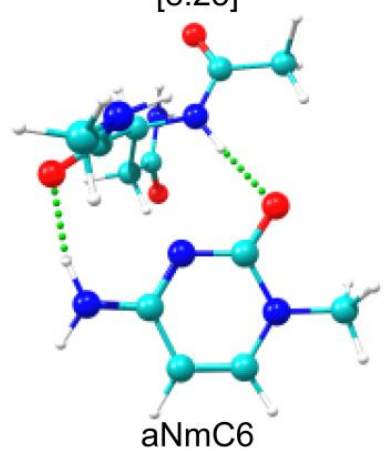

[11.61]

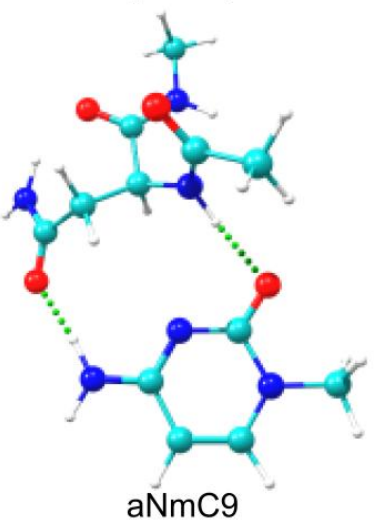

[13.46]

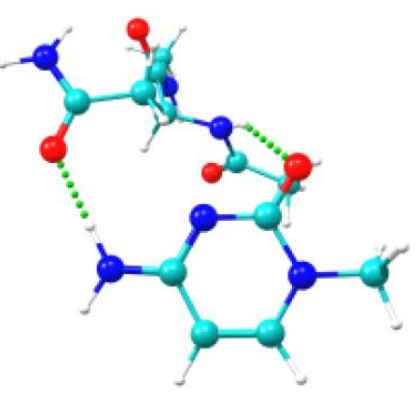

aNmC12

[14.25] 


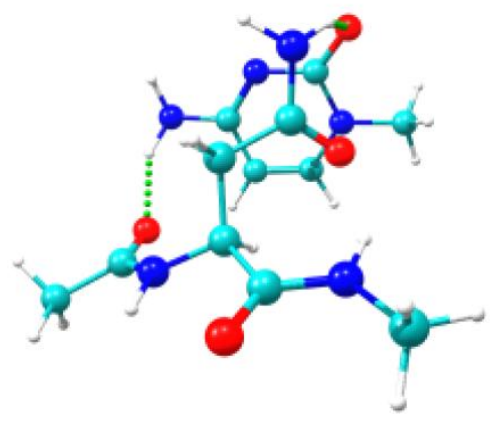

aNmC13

[15.33]

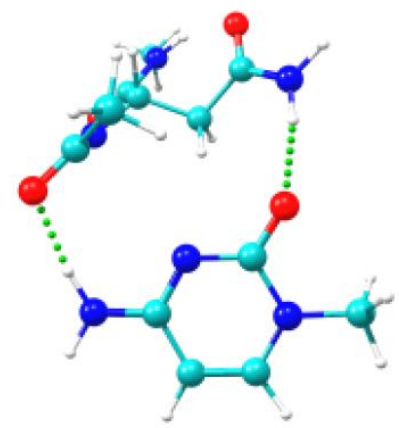

aNmC16

[17.26]

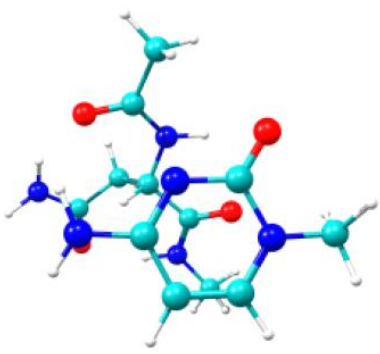

aNmC19

[18.25]

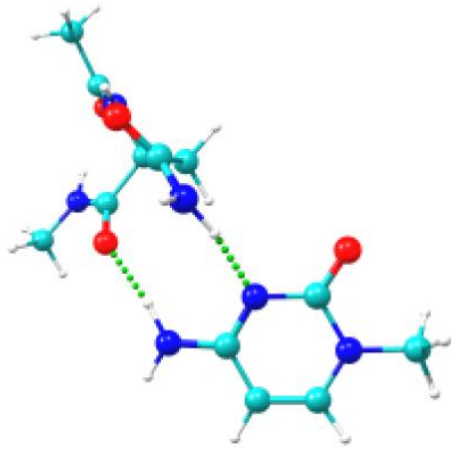

aNmC14

[15.38]

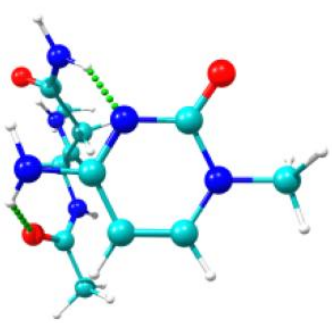

aNmC17

[17.93]

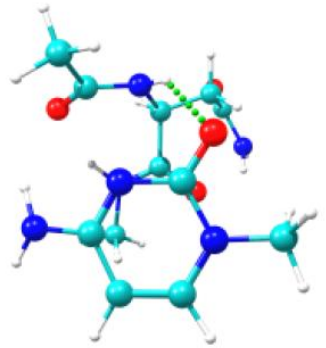

aNmC20

[20.14]

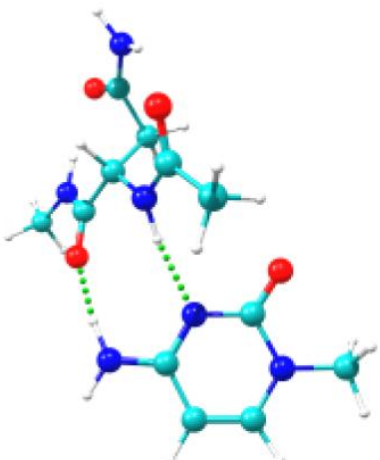

aNmC15

[15.91]

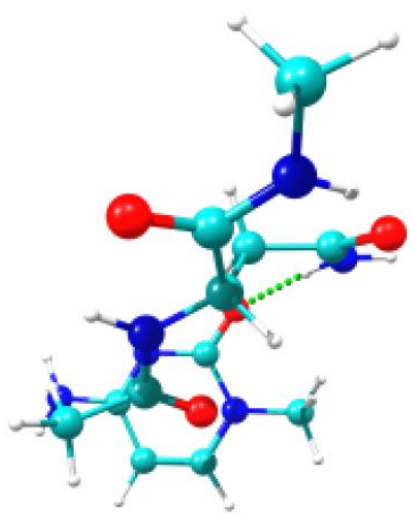

aNmC18

[18.10] 
Figure S05. The first 20 calculated structures for $a Q m C$ at $M 06-2 X / 6-311++G(d, p)$ level together with their relative stability $\left(\mathrm{kJ} \mathrm{mol}^{-1}\right)$ in brackets. ZPE correction was applied to the energy values.

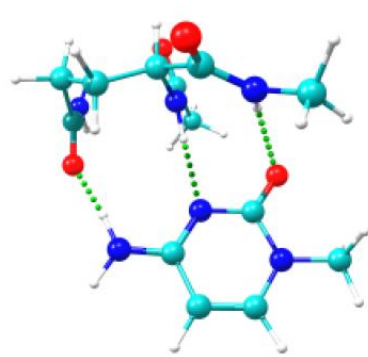

$\mathrm{aQmC1}$

[0.00]

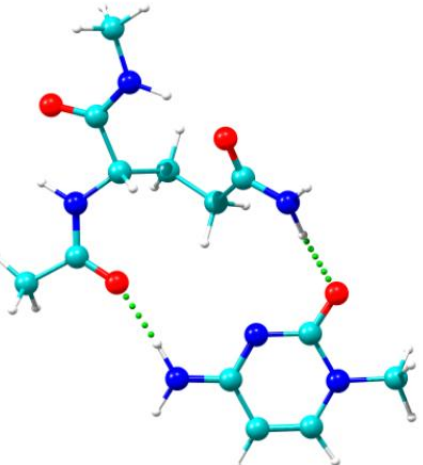

$\mathrm{aQmC4}$

[8.33]

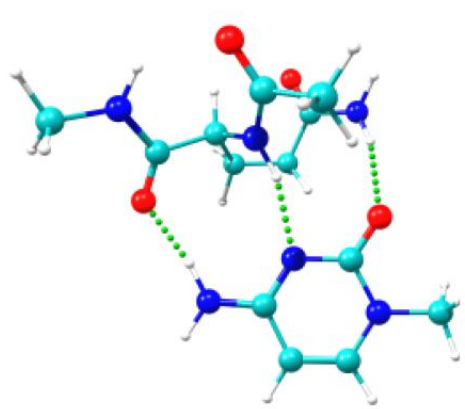

$\mathrm{aQmC7}$

[9.36]

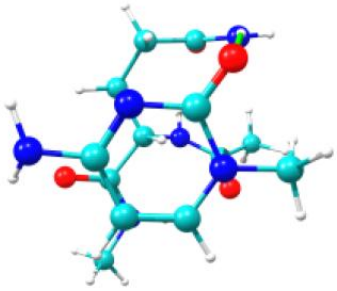

$\mathrm{aQmC10}$

[12.64]

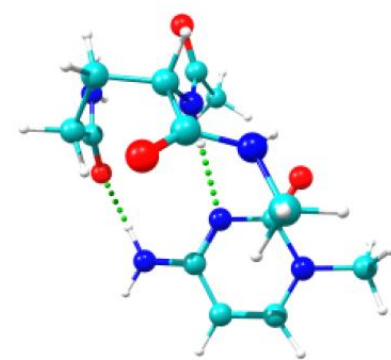

$\mathrm{aQmC2}$

[6.95]

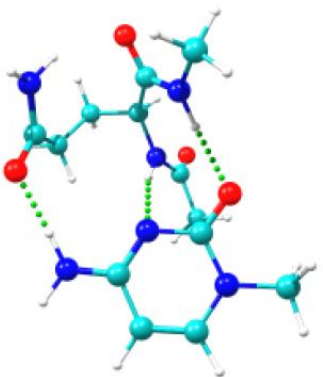

$\mathrm{aQmC5}$

[8.94]

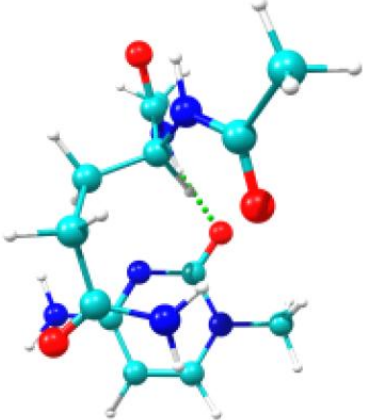

$\mathrm{aQmC8}$

[10.85]

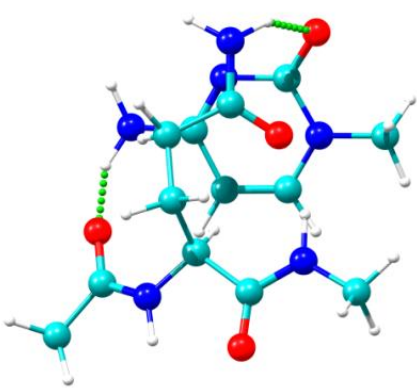

$\mathrm{aQmC} 11$

[13.68]

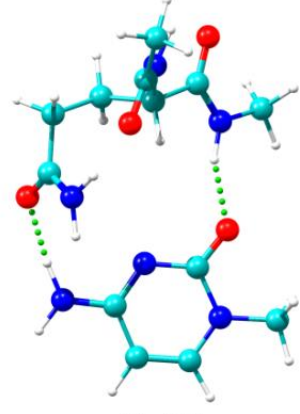

QmC3

[8.13]

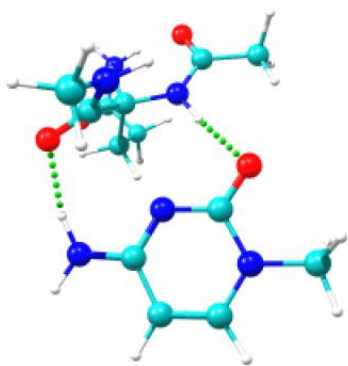

QmC6

[9.06]

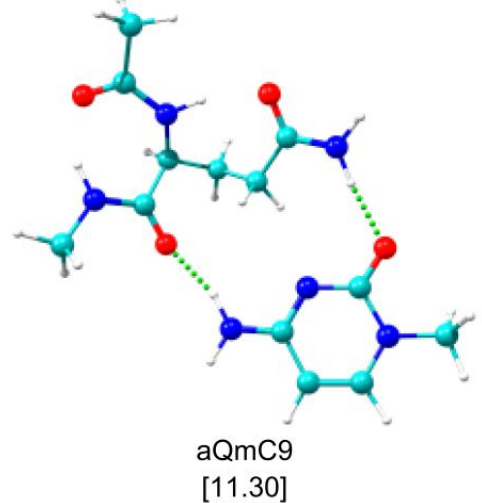

[11.30]

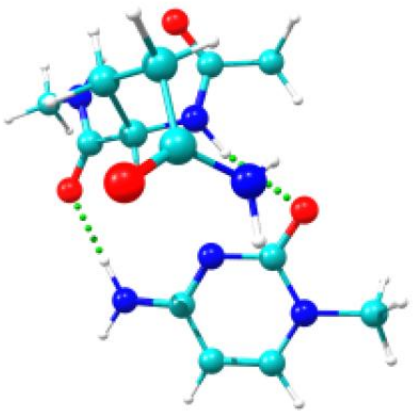

$\mathrm{aQmC} 12$

[13.88] 


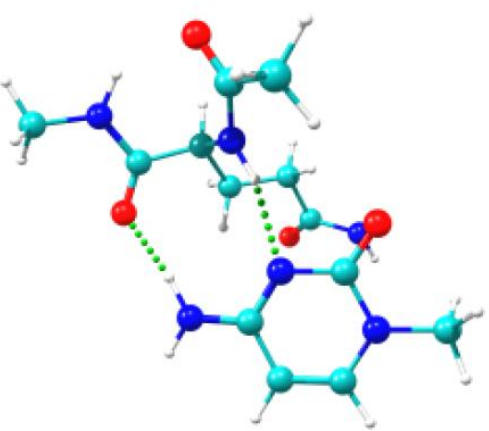

$\mathrm{aQmC13}$

[14.09]

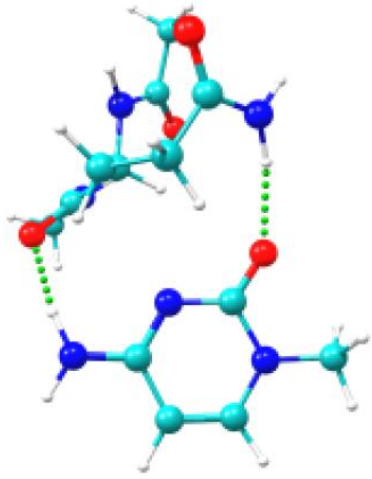

aQmC16

[16.56]

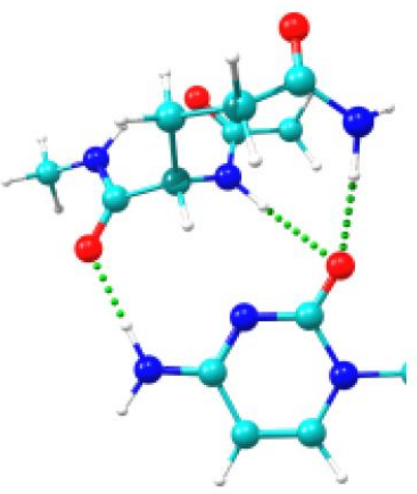

aQmC19

[18.28]

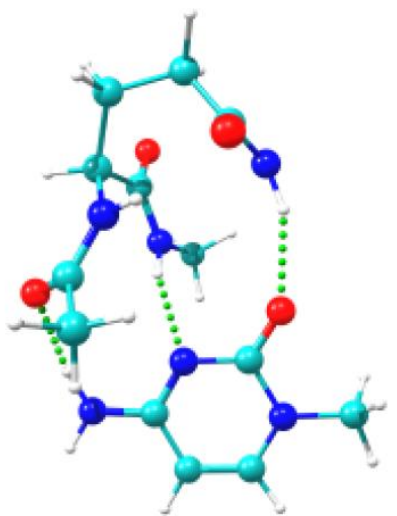

aQmC14

[14.29]

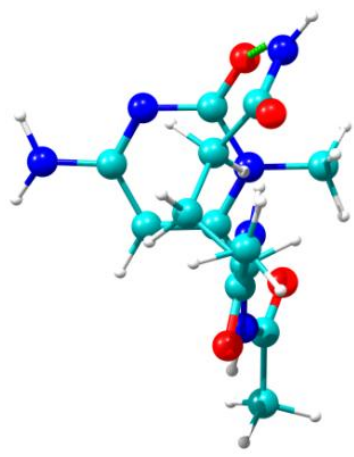

aQmC17

[17.31]

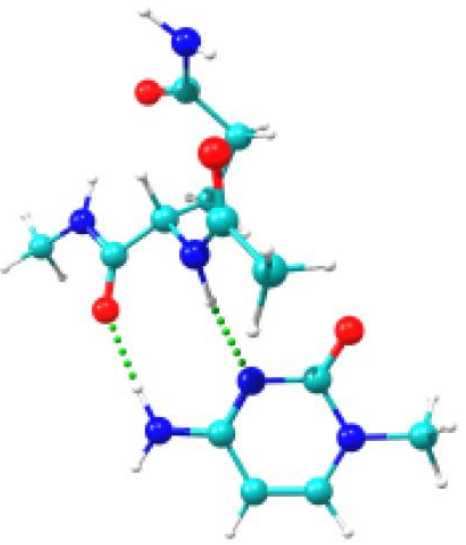

$\mathrm{aQmC20}$

[18.42]

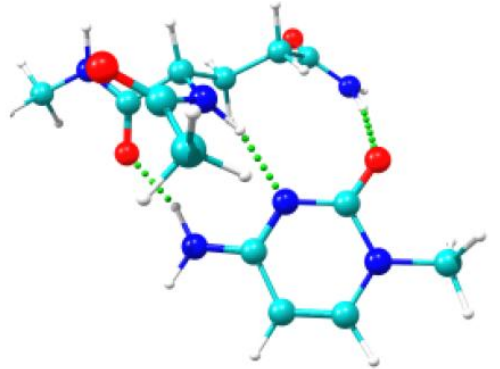

aQmC15

[15.01]

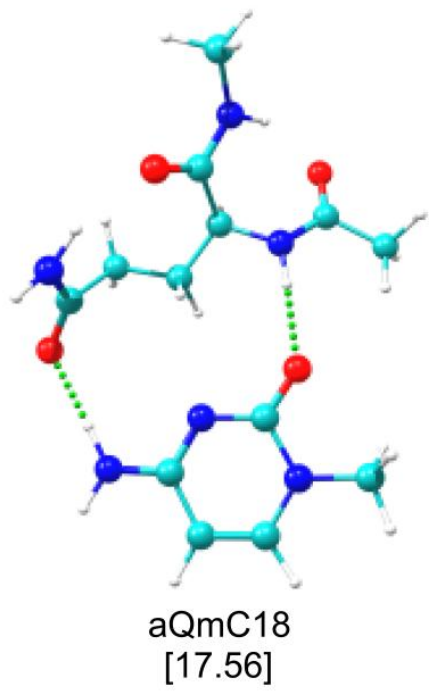


Figure S06. The first 20 calculated structures for $a R m C$ at $M 06-2 X / 6-311++G(d, p)$ level together with their relative stability $\left(\mathrm{kJ} \mathrm{mol}^{-1}\right)$ in brackets. ZPE correction was applied to the energy values.

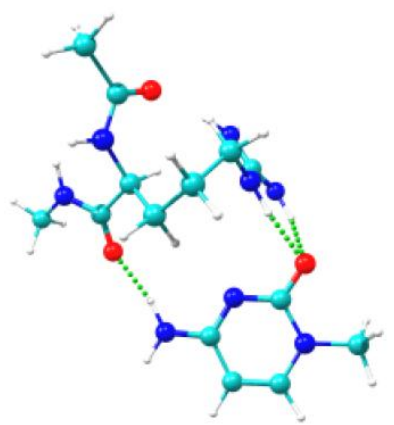

aRmC1

[0.00]
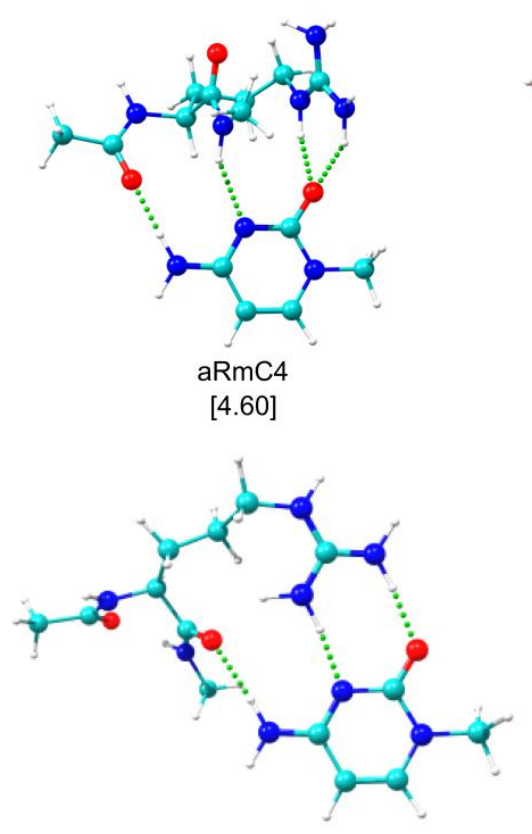

aRmC7

[6.98]

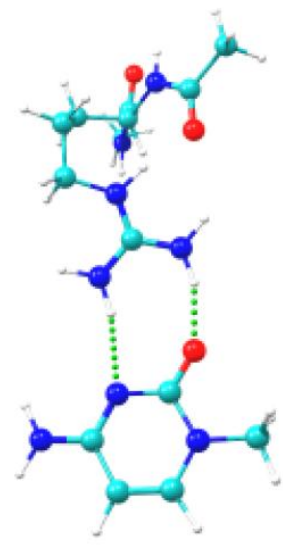

aRmC10

[10.64]
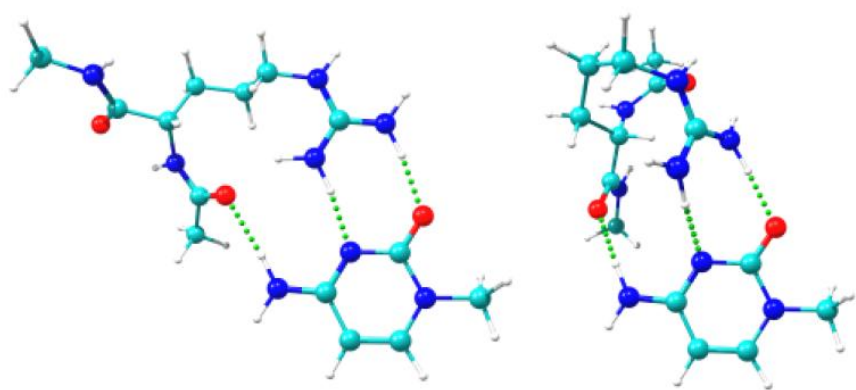

[0.20]

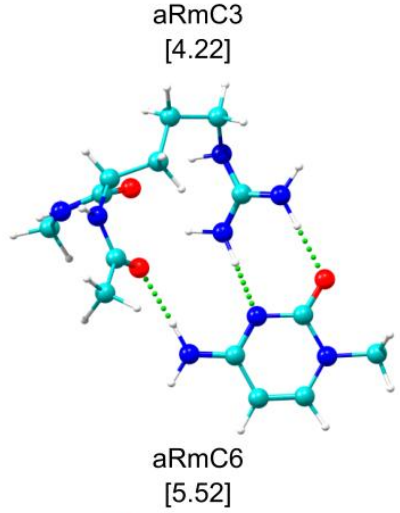

[5.16]

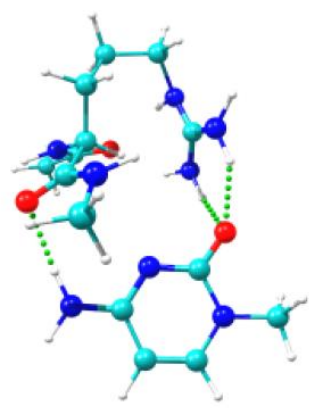

aRmC8

[8.70]

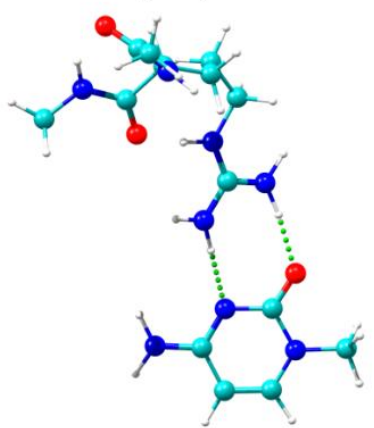

aRmC9

[9.01]

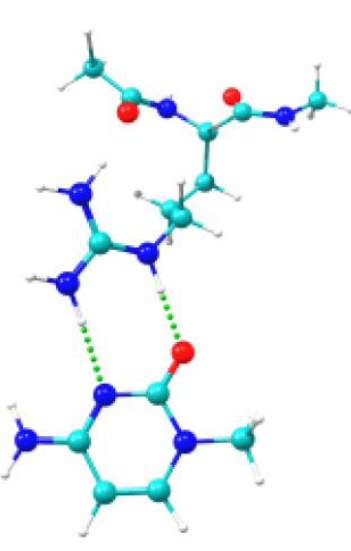

aRmC11

[11.46]

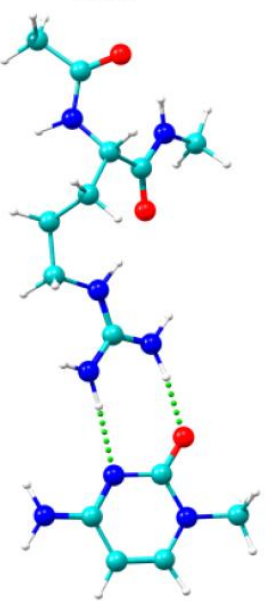

aRmC12

[12.12] 


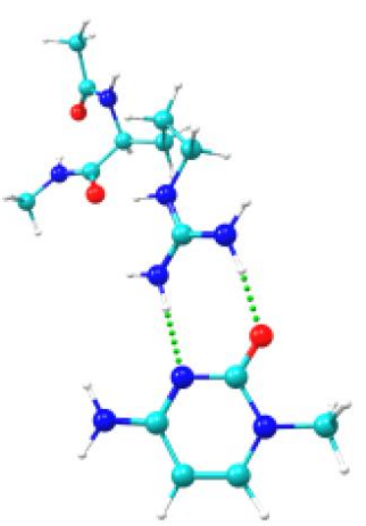

aRmC13

[12.65]

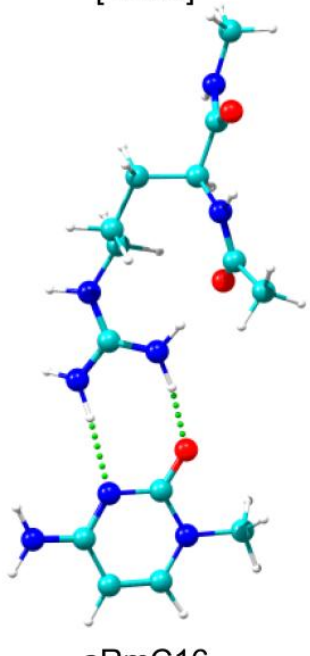

[13.94]

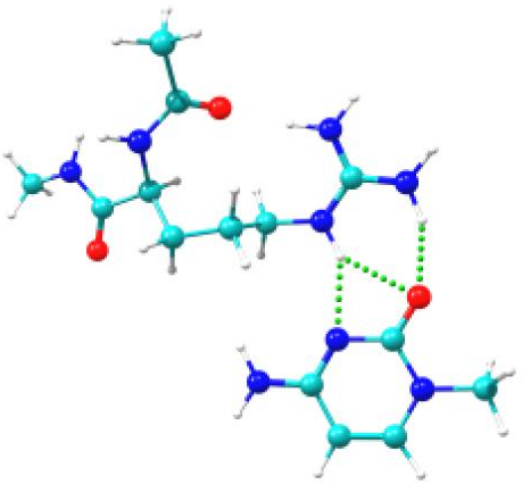

aRmC19

[16.30]

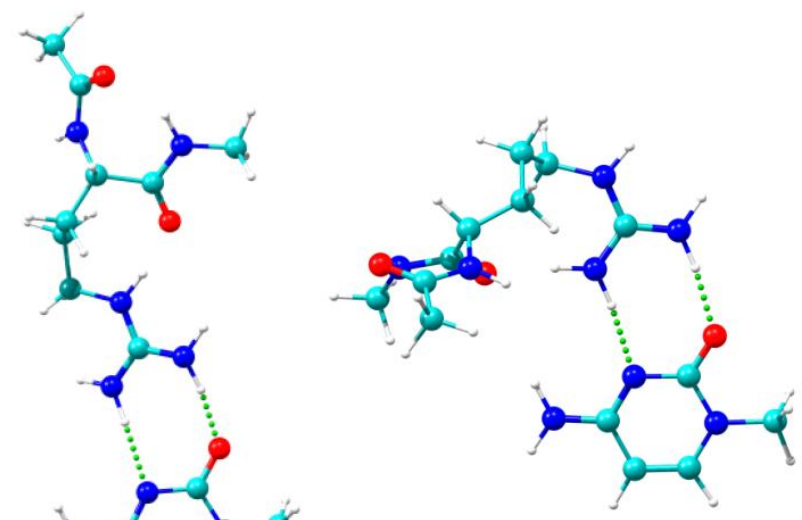

aRmC15

[13.44]

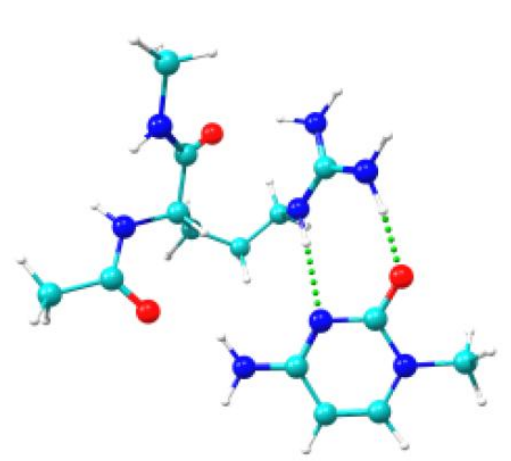

aRmC17

[14.23]

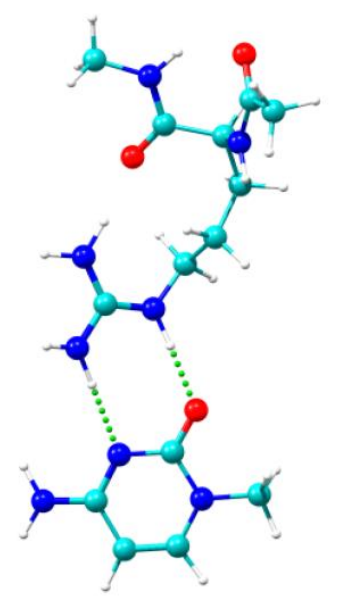

aRmC20

[16.80]

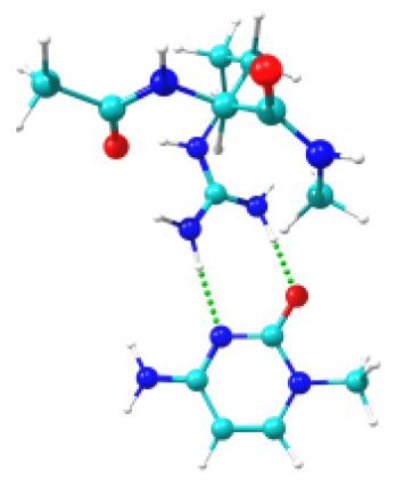

aRmC18

[16.21] 
Figure S07. The first 20 calculated structures for aAmT at M06-2X/6-311++G(d,p) level together with their relative stability $\left(\mathrm{kJ} \mathrm{mol}^{-1}\right)$ in brackets. ZPE correction was applied to the energy values.

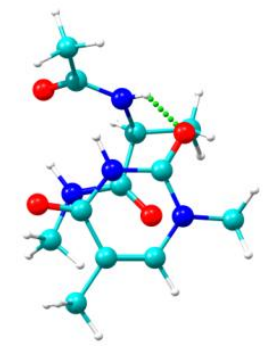

aAmT1

[0.00]

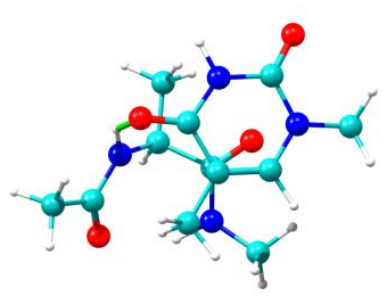

aAmT4

[4.48]

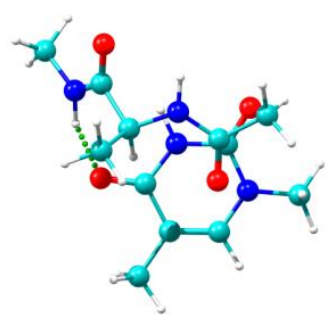

aAmT7

[5.63]

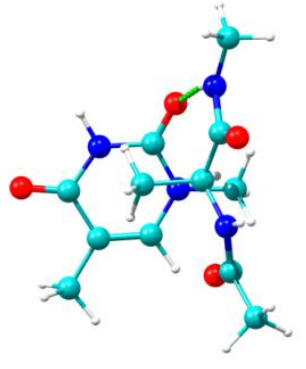

aAmT10

[7.87]

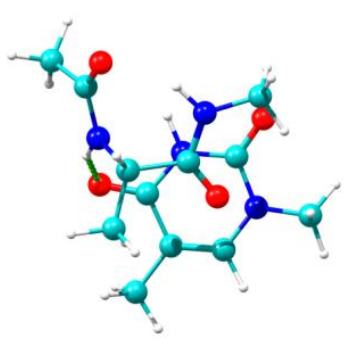

aAmT2

[2.35]

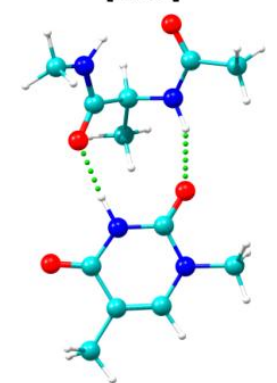

aAmT5

[5.37]

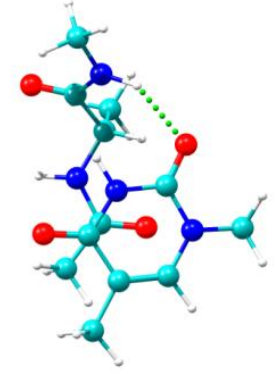

aAmT8

[7.07]

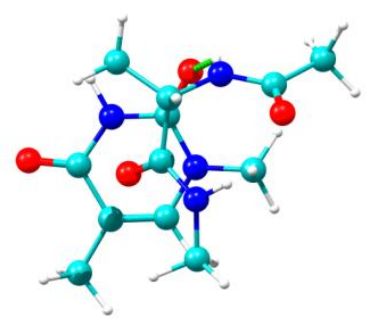

aAmT11

[8.69]

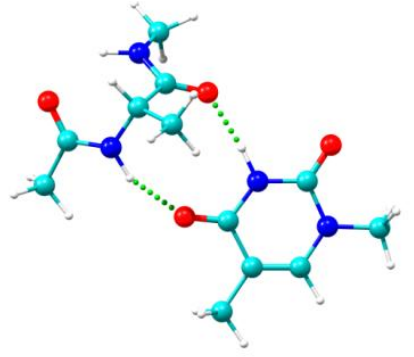

aAmT3

[3.36]

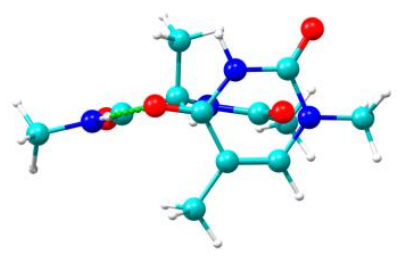

aAmT6

[5.62]

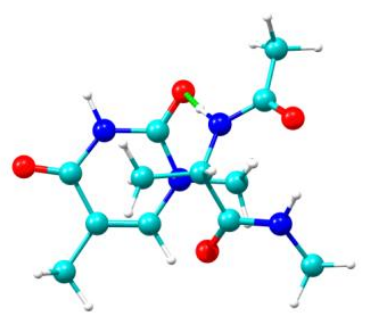

aAmT9

[7.56]

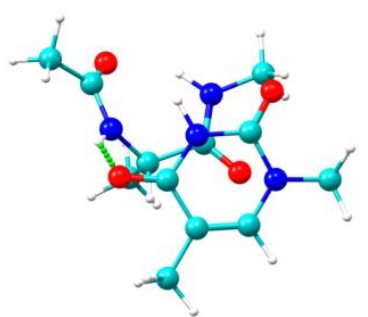

aAmT12

[9.74] 


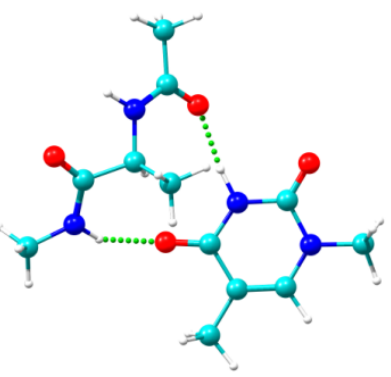

aAmT13

[11.44]

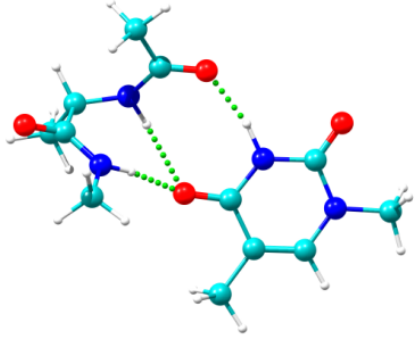

aAmT16

[14.50]

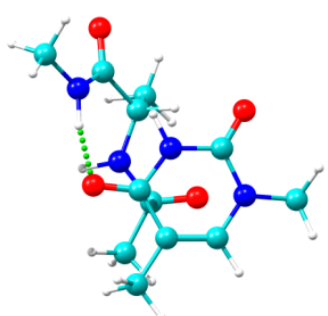

aAmT19

[15.01]

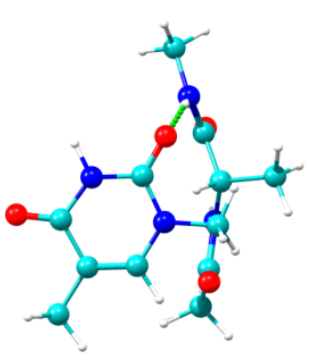

aAmT14

[11.50]

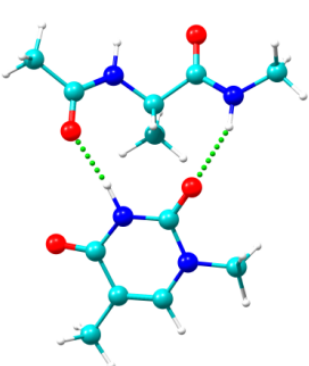

aAmT17

[14.75]

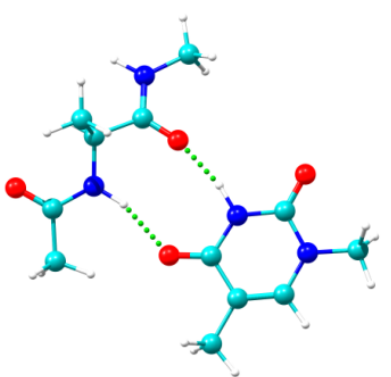

aAmT20

[15.15]

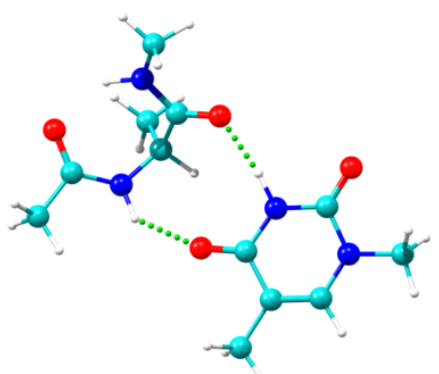

aAmT15

[13.53]

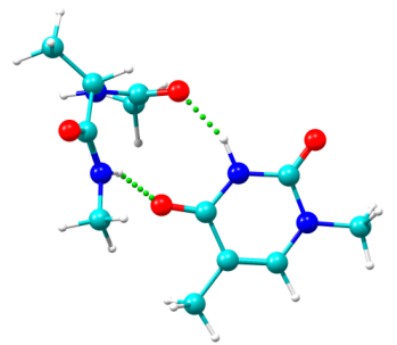

aAmT18

[14.92] 
Figure S08. The first 20 calculated structures for $\mathrm{aVmT}$ at $\mathrm{M} 06-2 \mathrm{X} / 6-311++\mathrm{G}(\mathrm{d}, \mathrm{p})$ level together with their relative stability $\left(\mathrm{kJ} \mathrm{mol}^{-1}\right)$ in brackets. ZPE correction was applied to the energy values.

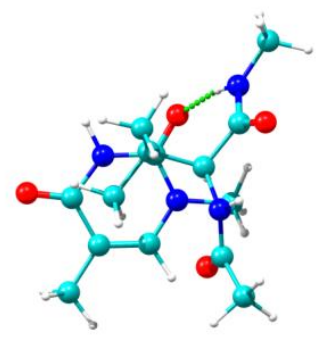

$\mathrm{aVmT1}$

[0.00]

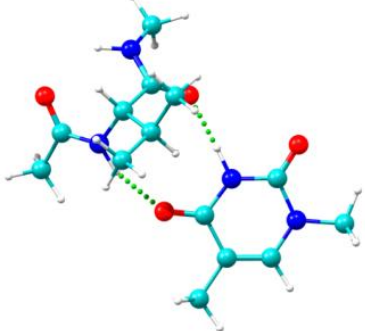

$\mathrm{aVmT4}$

[2.75]

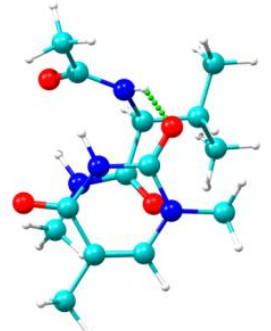

aVmT7

[3.12]

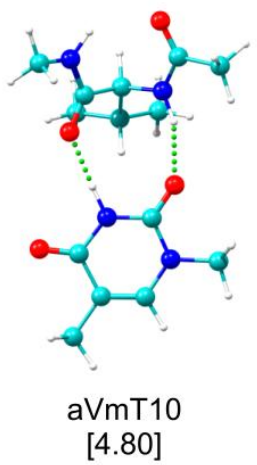

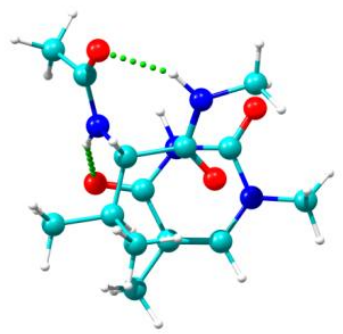

$\mathrm{aVmT2}$

[0.41]

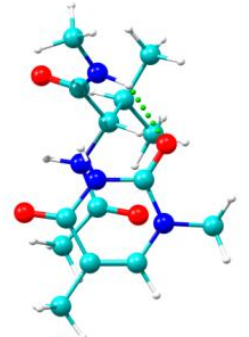

$\mathrm{aVmT5}$

[2.91]

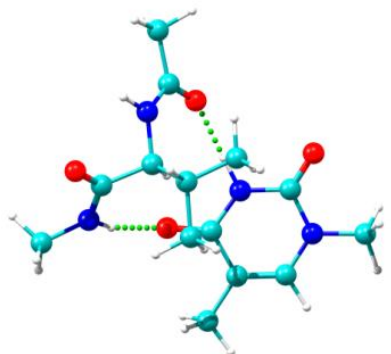

aVmT8

[3.70]

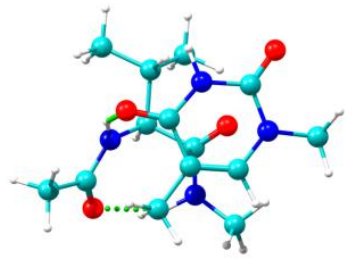

$\mathrm{aVmT11}$

[5.40]

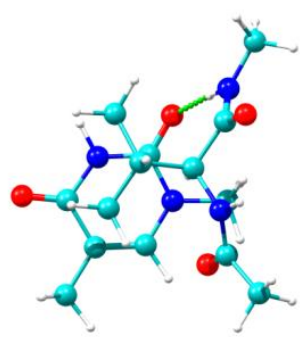

$\mathrm{aVmT3}$

[2.72]

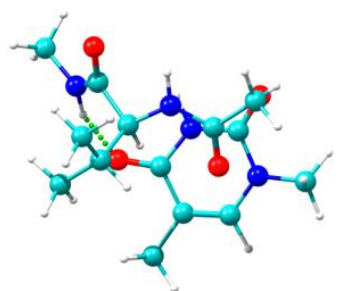

aVmT6

[2.96]

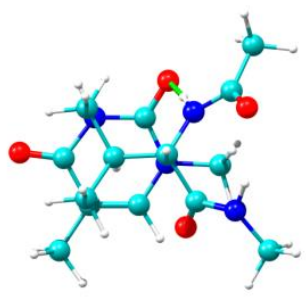

aVmT9

[4.39]

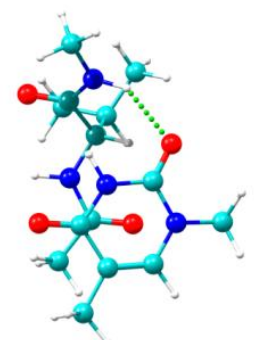

$\mathrm{aVmT12}$

[6.21] 


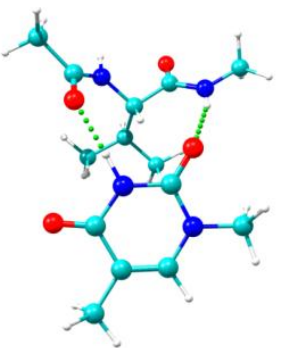

$\mathrm{aVmT13}$

[6.77]

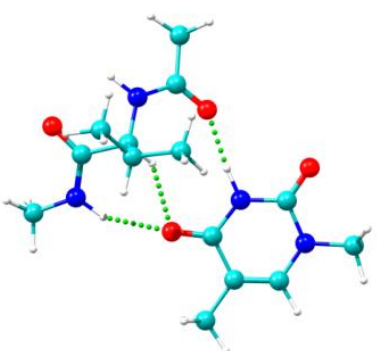

aVmT16

[7.24]

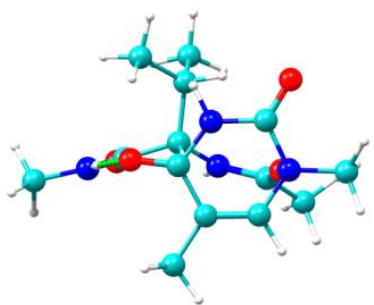

aVmT19

[10.15]

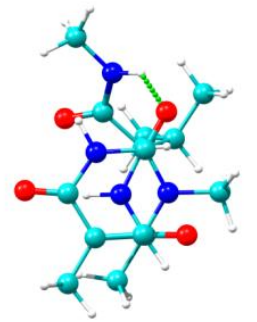

$\mathrm{aVmT14}$

[6.91]

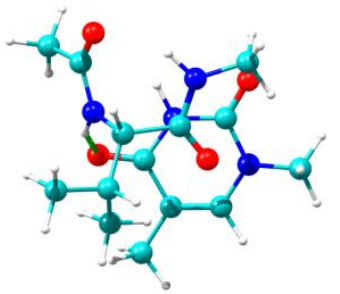

$\mathrm{aVmT17}$

[7.97]

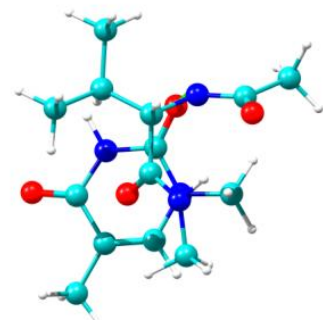

$\mathrm{aVmT20}$

[10.16]

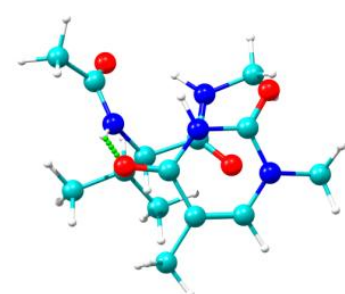

aVmT15

[7.13]

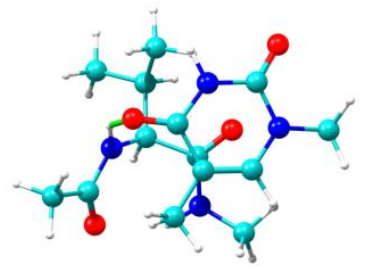

$\mathrm{aVmT18}$

[8.02] 
Figure S09. The first 20 calculated structures for aImT at M06-2X/6-311++G(d,p) level together with their relative stability $\left(\mathrm{kJ} \mathrm{mol}^{-1}\right)$ in brackets. ZPE correction was applied to the energy values.

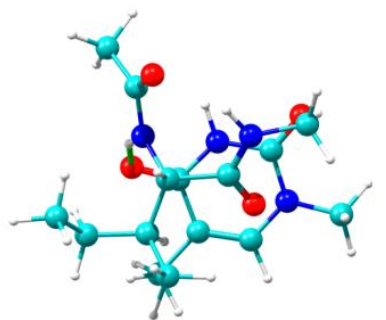

almT1

[0.00]

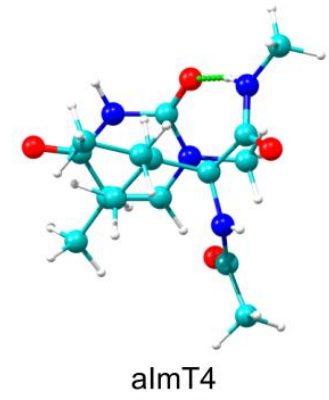

[3.02]

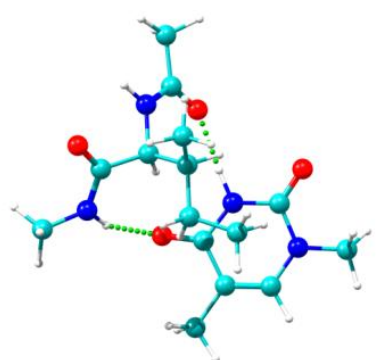

almT7

[4.32]

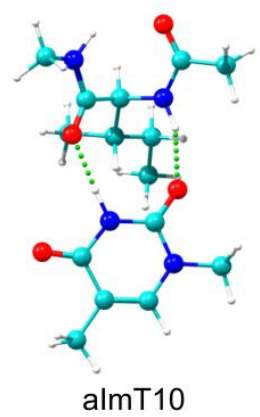

[5.81]

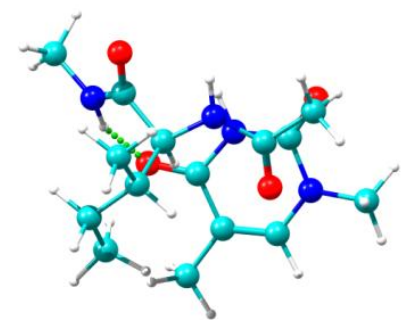

almT2

[0.51]

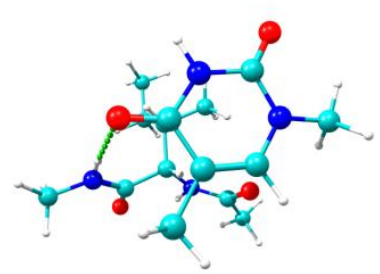

almT5

[3.74]

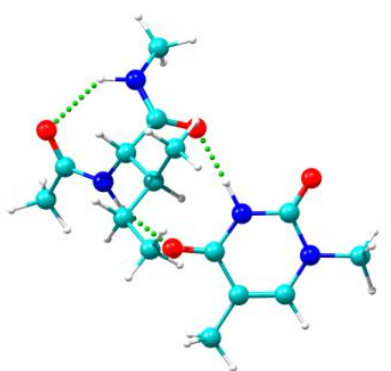

almT8

[4.47]

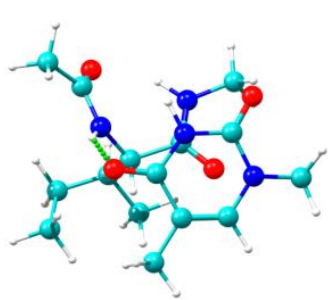

almT11

[6.79]

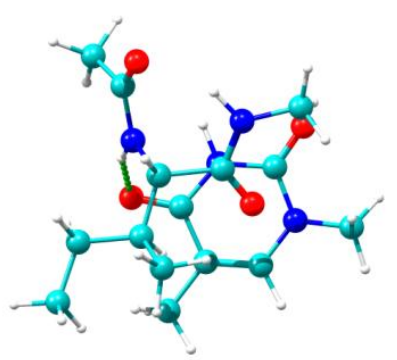

almT3

[2.11]

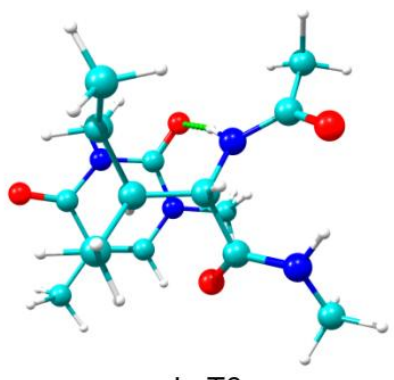

almT6

[4.13]

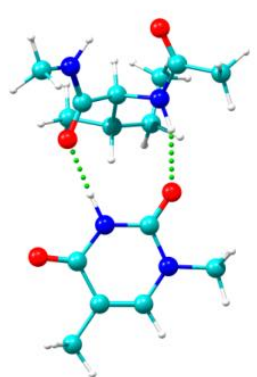

almT9

[5.07]

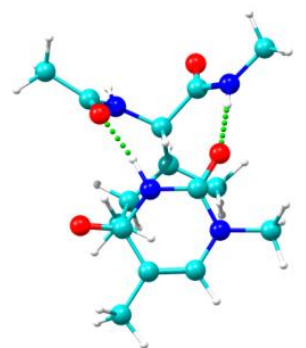

almT12

[7.37] 


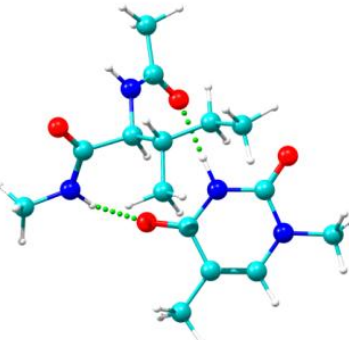

almT13

[8.28]

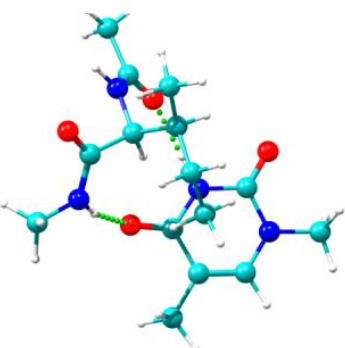

almT16

[8.87]

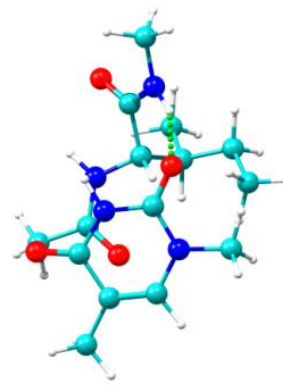

almT19

[9.64]

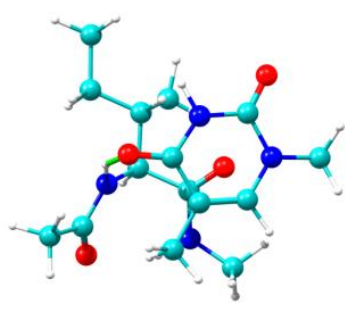

almT14

[8.71]

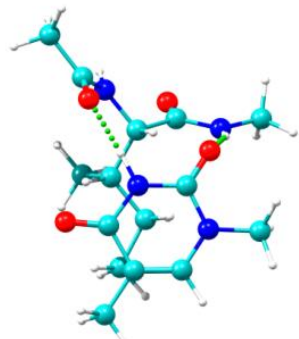

almT17

[8.90]

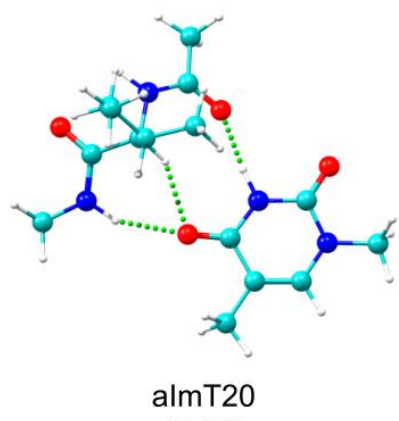

[9.89]

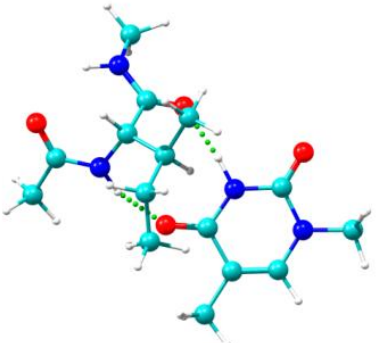

almT15

[8.82]

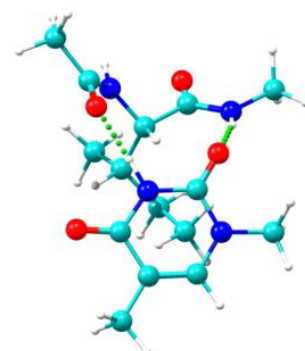

almT18

[9.47] 
Figure S10. The first 20 calculated structures for aNmT at M06-2X/6-311++G(d,p) level together with their relative stability $\left(\mathrm{kJ} \mathrm{mol}^{-1}\right)$ in brackets. ZPE correction was applied to the energy values.

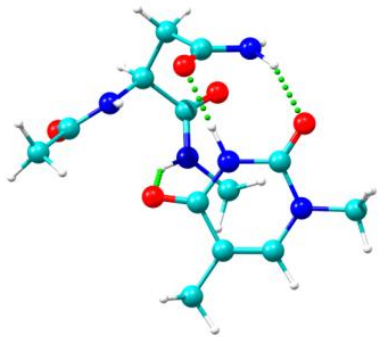

aNmT1

[0.00]

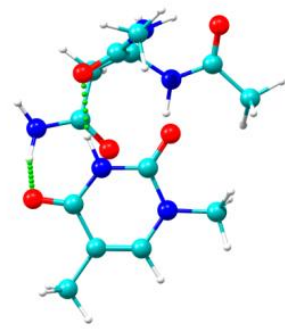

aNmT4

[1.59]

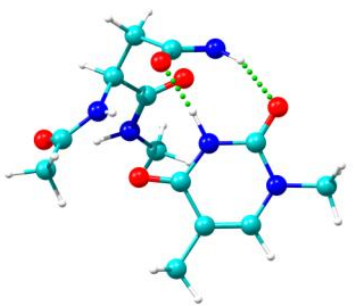

aNmT7

[4.18]

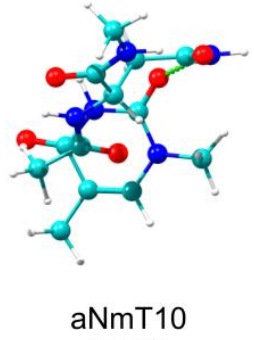

[5.96]

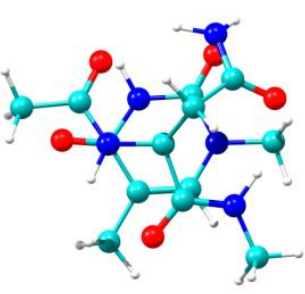

aNmT2

[0.36]

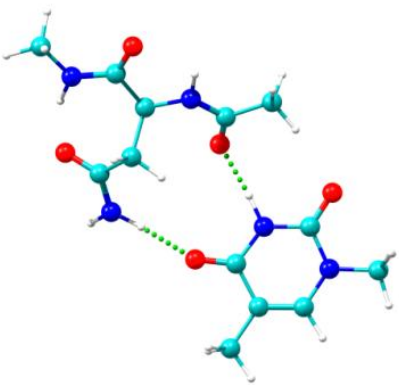

aNmT5

[3.73]

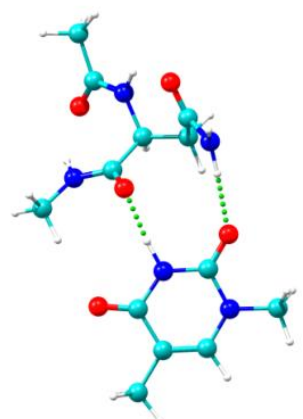

aNmT8

[5.63]

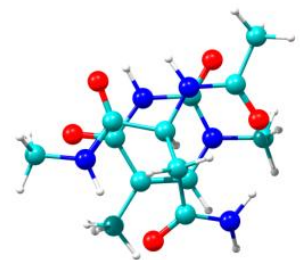

aNmT11

[6.00]

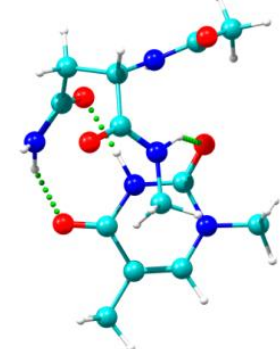

aNmT3

[0.90]

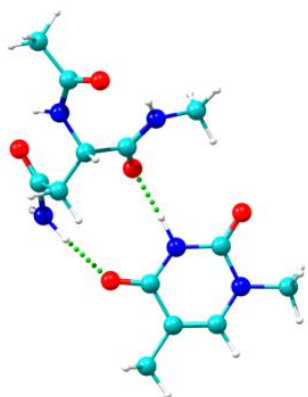

aNmT6

[4.13]

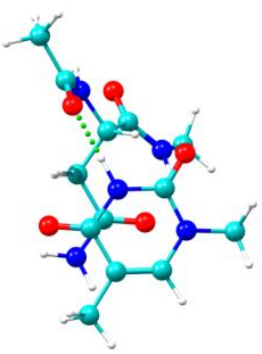

aNmT9

[5.66]

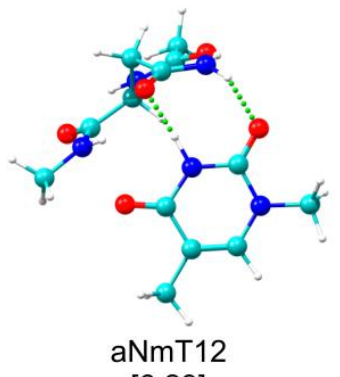

[6.29] 


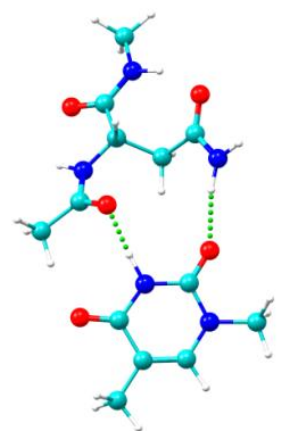

aNmT13

[6.37]

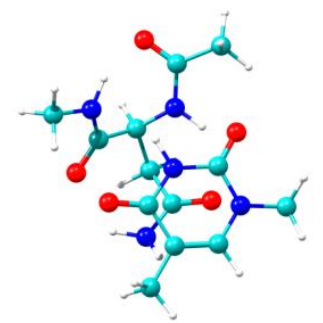

aNmT16

[7.43]

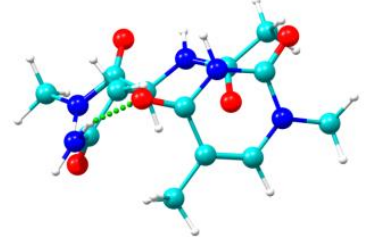

aNmT19

[8.79]

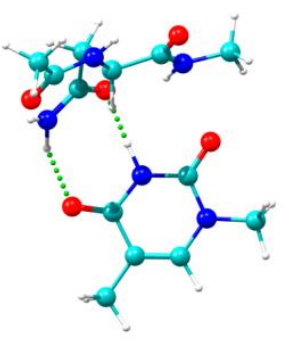

aNmT14

[6.44]

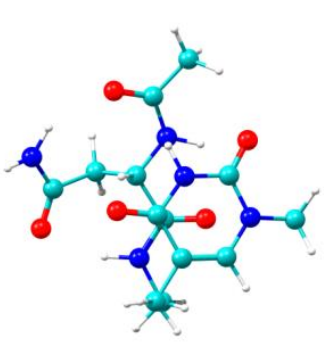

aNmT17

[8.04]

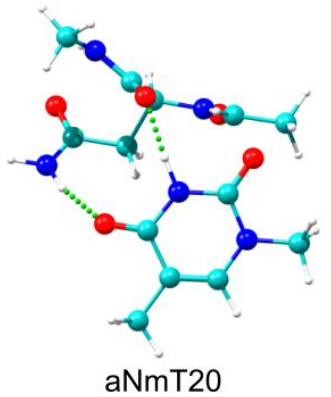

[9.75]

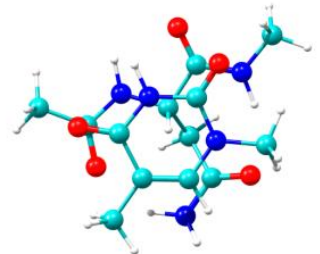

aNmT15

[7.15]

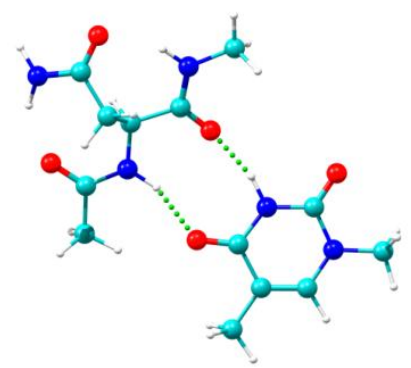

aNmT18

[8.30] 
Figure S11. The first 20 calculated structures for $\mathrm{aQmT}$ at M06-2X/6-311++G(d,p) level together with their relative stability $\left(\mathrm{kJ} \mathrm{mol}^{-1}\right)$ in brackets. ZPE correction was applied to the energy values.

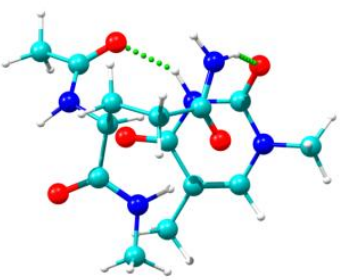

$\mathrm{aQmT1}$

[0.00]

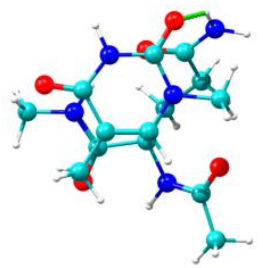

$\mathrm{aQmT4}$

[4.32]

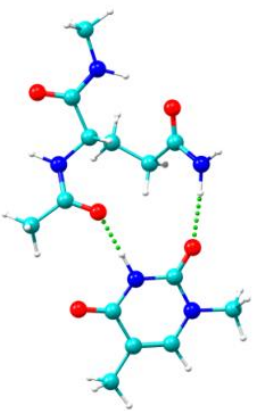

$\mathrm{aQmT7}$

[9.44]

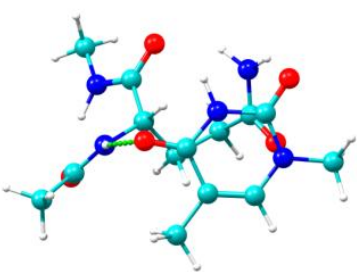

$\mathrm{aQmT10}$

[12.64]

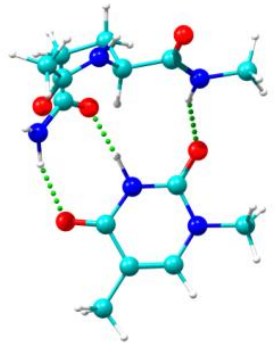

aQmT2

[3.35]

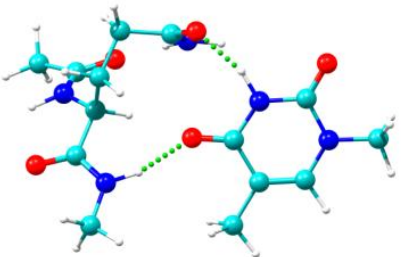

aQmT5

[6.59]

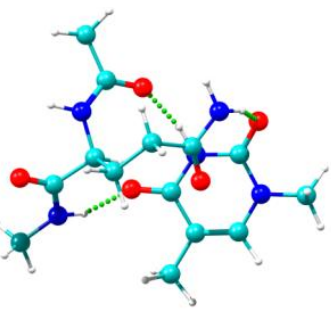

aQmT8

[9.62]

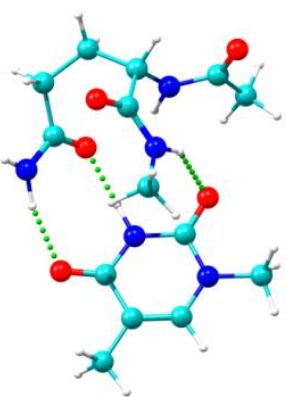

aQmT11

[12.76]

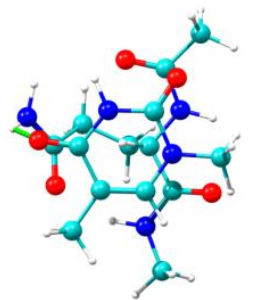

aQmT3

[3.65]
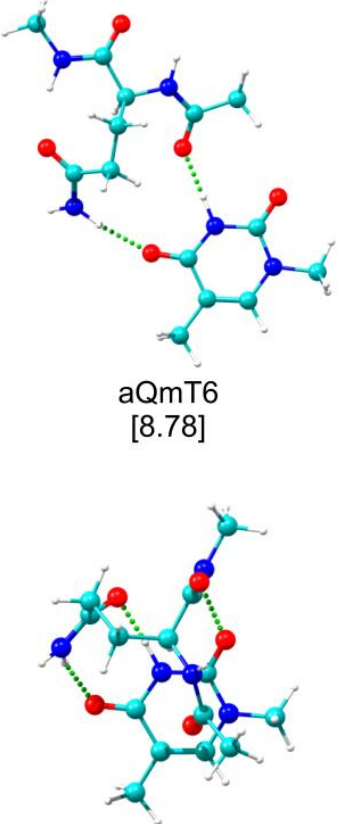

aQmT9

[9.83]

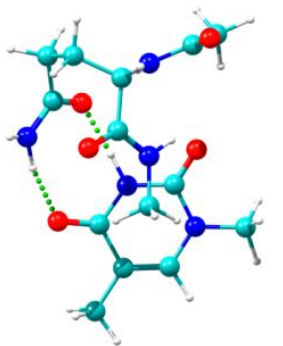

aQmT12

[13.26] 


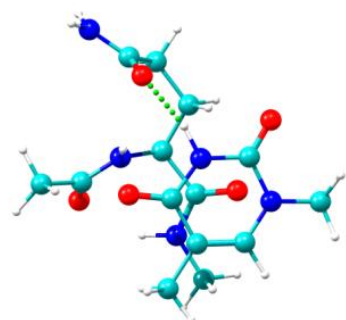

aQmT13

[13.73]

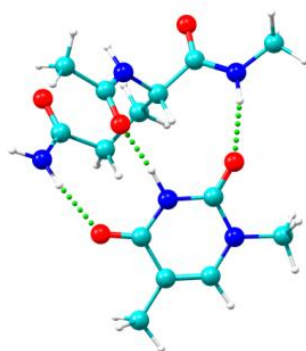

aQmT16

[14.84]

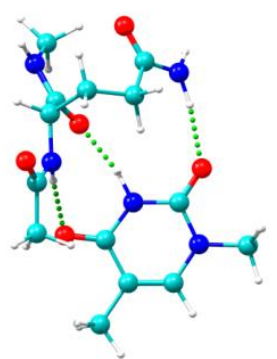

aQmT19

[16.20]

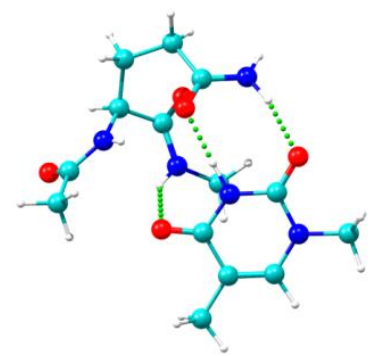

aQmT14

[13.79]

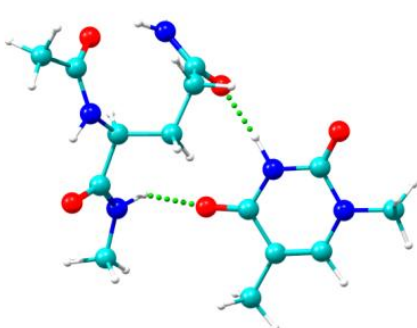

aQmT17

[14.96]

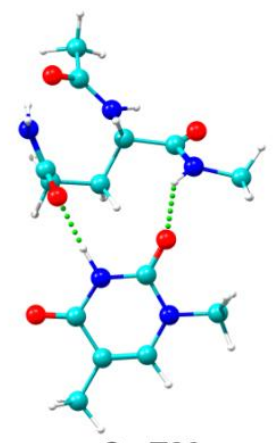

aQmT20

[17.19]

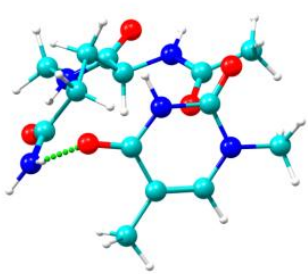

aQmT15

[14.33]

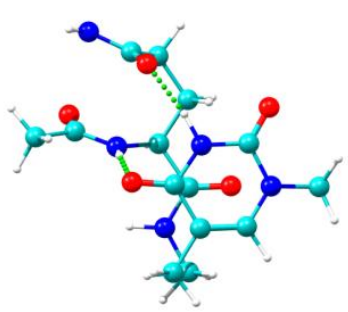

aQmT18

[15.12] 
Figure S12. The first 20 calculated structures for $a R m T$ at $M 06-2 X / 6-311++G(d, p)$ level together with their relative stability $\left(\mathrm{kJ} \mathrm{mol}^{-1}\right)$ in brackets. ZPE correction was applied to the energy values.

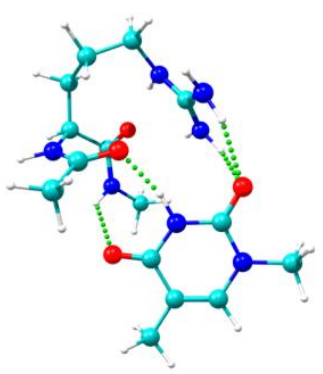

aRmT1

[0.00]

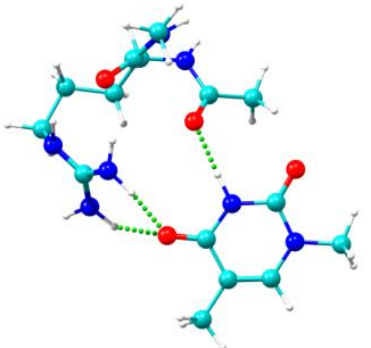

aRmT4

[11.89]

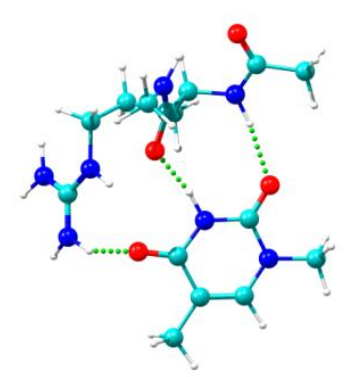

aRmT7

[12.88]

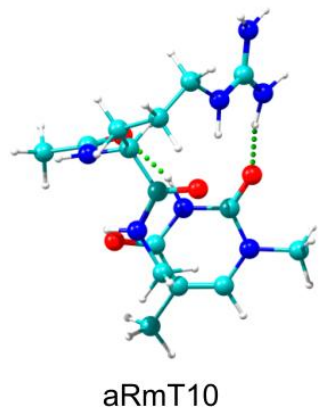

[13.78]

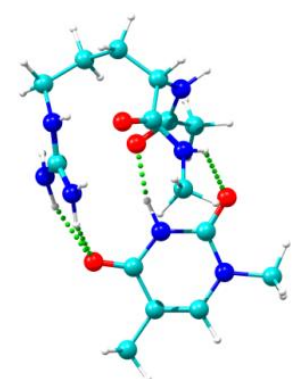

aRmT2

[0.96]

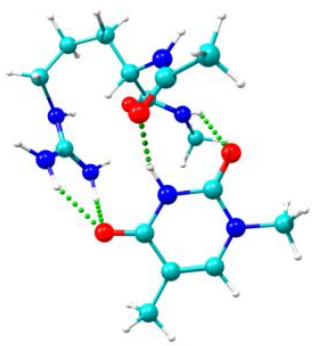

aRmT5

[12.77]

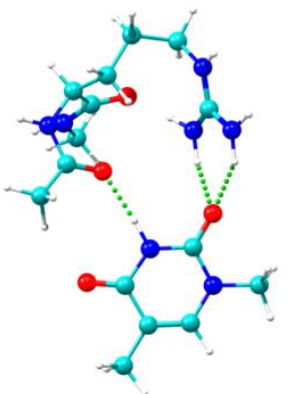

aRmT8

[13.21]

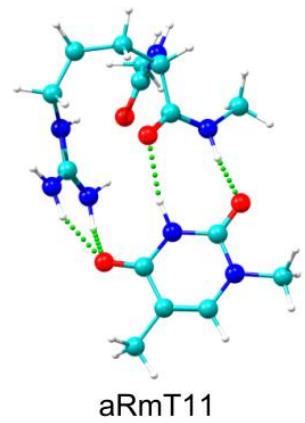

[14.61]

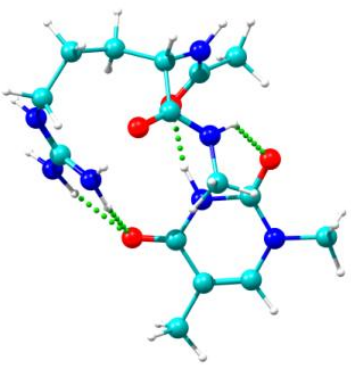

aRmT3

[9.87]

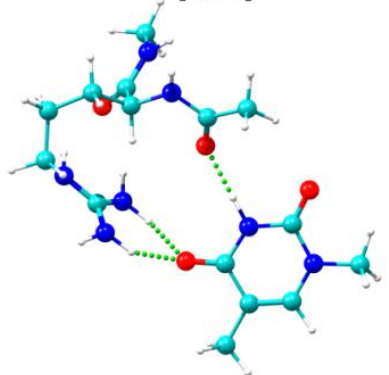

aRmT6

[12.87]

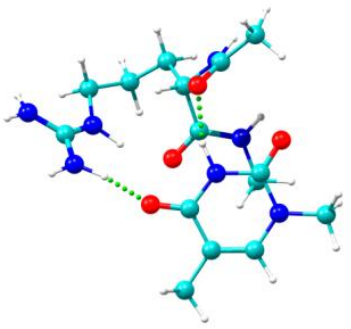

aRmT9

[13.29]

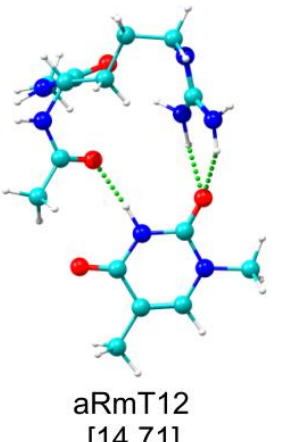




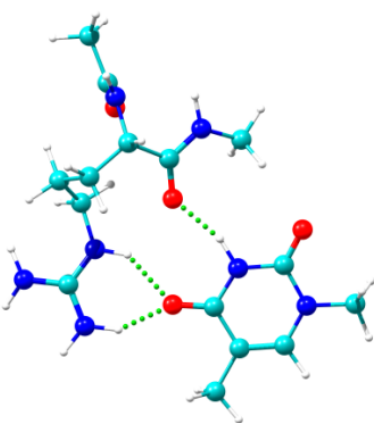

aRmT13

[15.02]

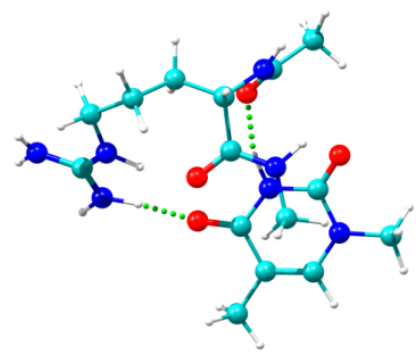

aRmT16

[15.52]

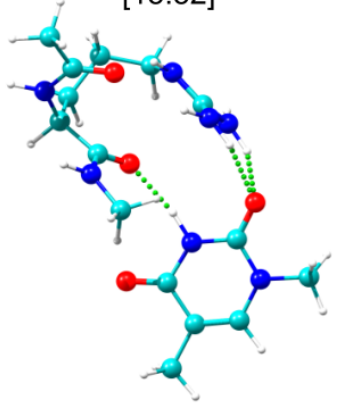

aRmT19

[18.52]

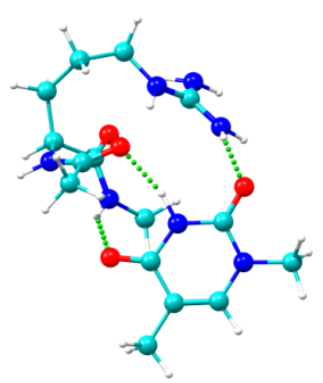

aRmT14

[15.15]

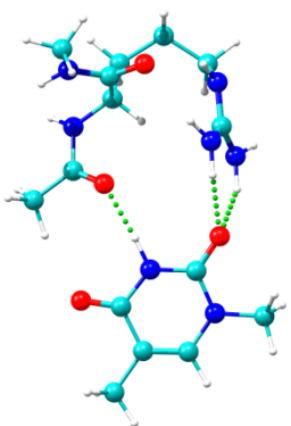

aRmT17

[16.35]

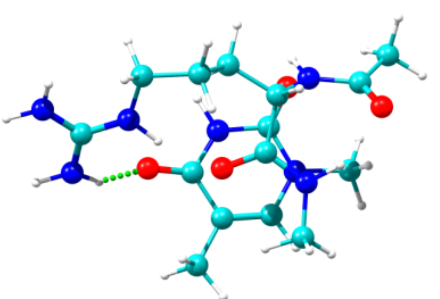

aRmT20

[20.42]

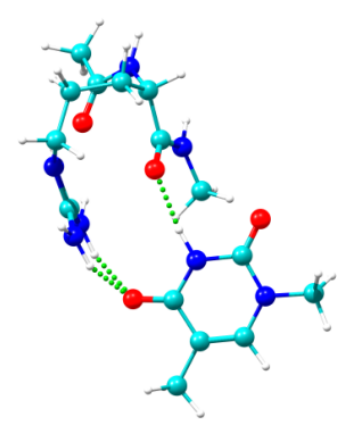

aRmT15

[15.34]

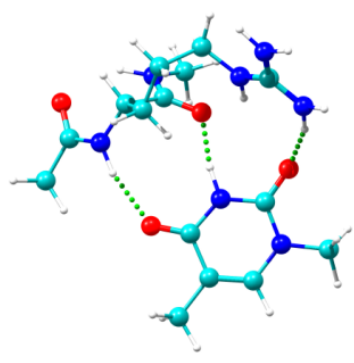

aRmT18

[17.27] 
Figure S13. The first 20 calculated structures for aAmA at M06-2X/6-311++G(d,p) level together with their relative stability $\left(\mathrm{kJ} \mathrm{mol}^{-1}\right)$ in brackets. ZPE correction was applied to the energy values.

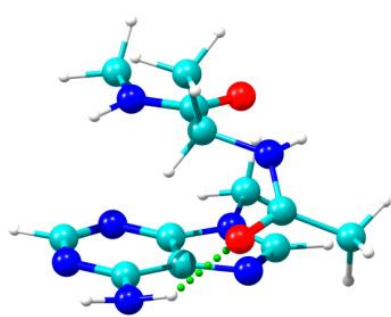

aAmA1

[0.00]

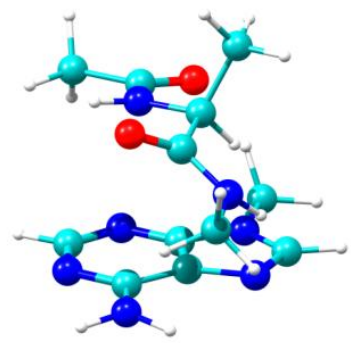

aAmA4

[6.24]

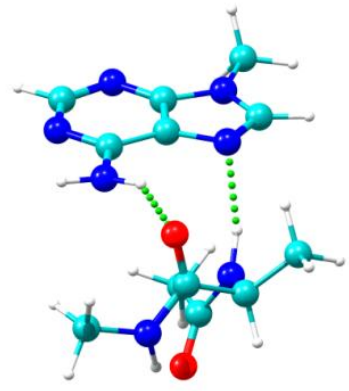

aAmA7

[9.15]

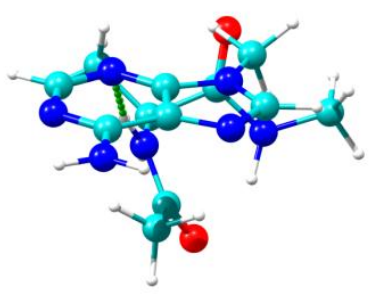

aAmA10

[11.26]

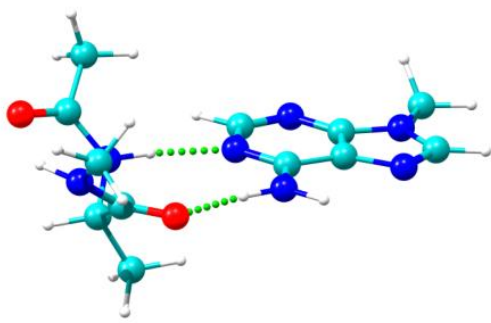

aAmA2

[0.94]

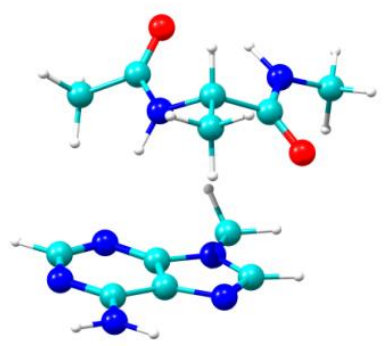

aAmA5

[6.59]

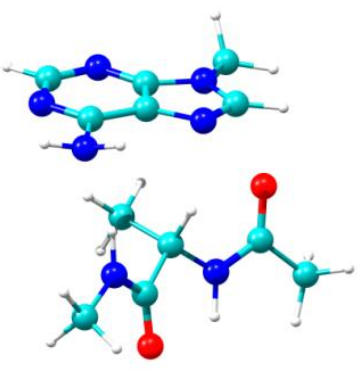

aAmA8

[9.73]

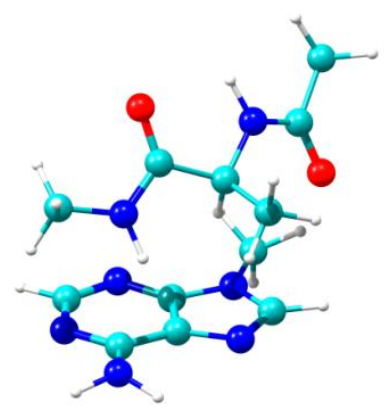

aAmA11

[12.37]

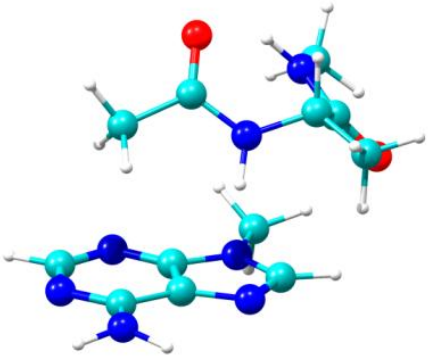

aAmA3

[5.79]

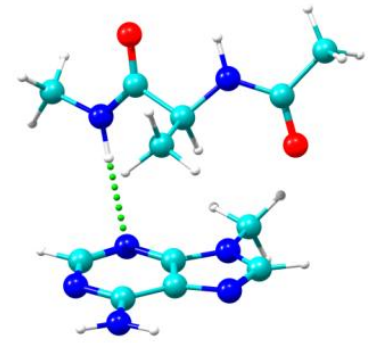

aAmA6

[7.55]

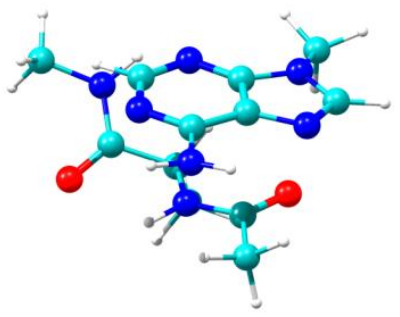

aAmA9

[10.10]

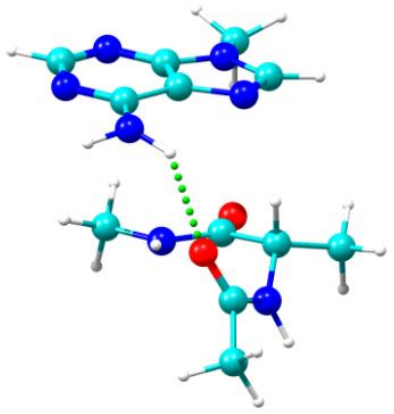

aAmA12

[15.00] 


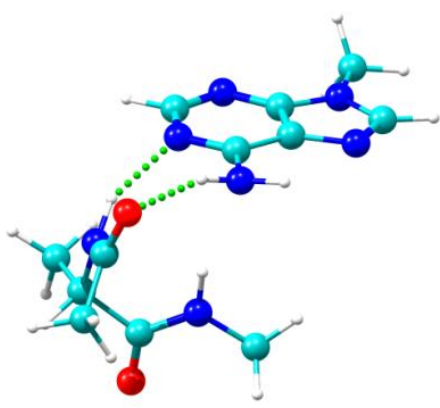

aAmA13

[15.22]

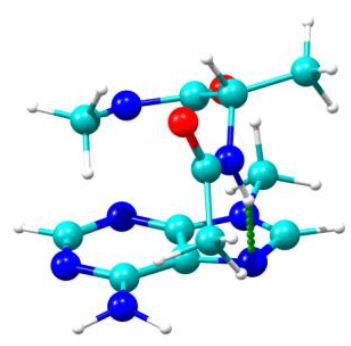

aAmA16

[18.85]

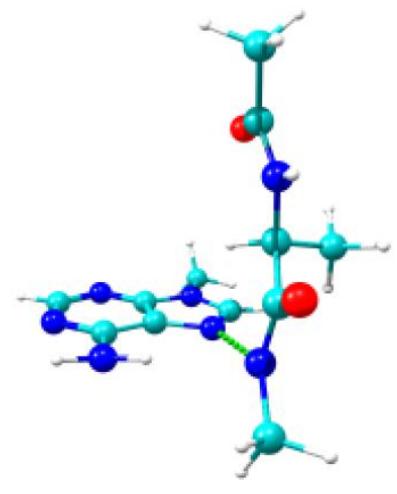

aAmA19

[22.32]

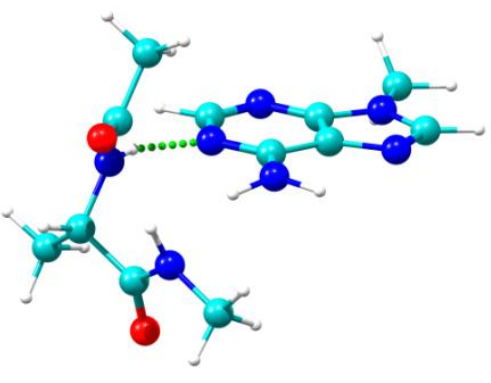

aAmA14

[17.13]

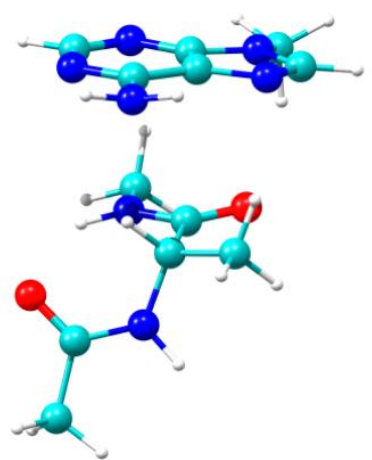

aAmA17

[19.46]
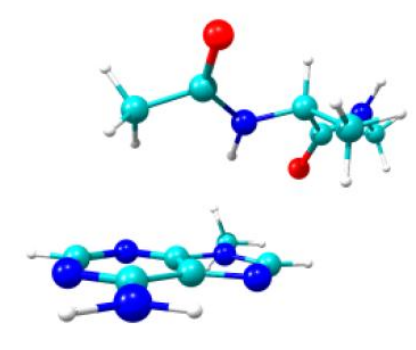

aAmA20
[22.63]

aAmA20
[22.63]

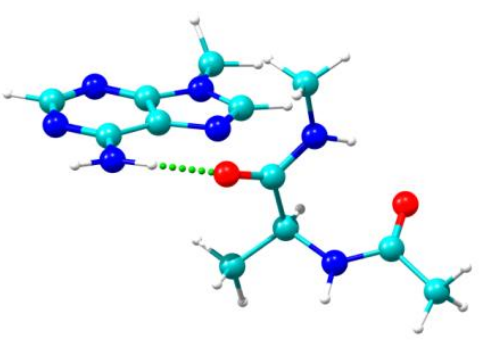

aAmA15

[18.60]

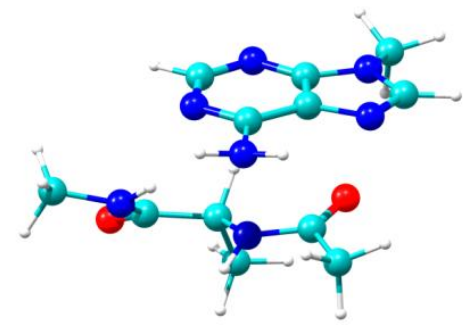

aAmA18

[21.04] 
Figure S14. The first 20 calculated structures for $\mathrm{aVmA}$ at $\mathrm{M} 06-2 \mathrm{X} / 6-311++\mathrm{G}(\mathrm{d}, \mathrm{p})$ level together with their relative stability $\left(\mathrm{kJ} \mathrm{mol}^{-1}\right)$ in brackets. ZPE correction was applied to the energy values.

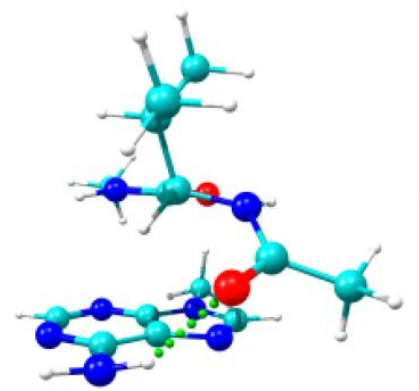

aVmA1

[0.00]

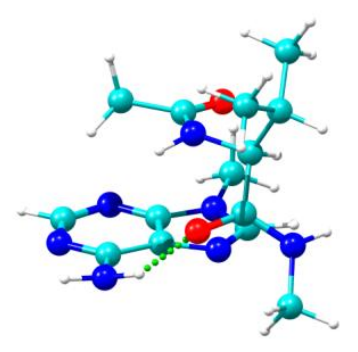

$\mathrm{aVmA} 4$

[7.22]

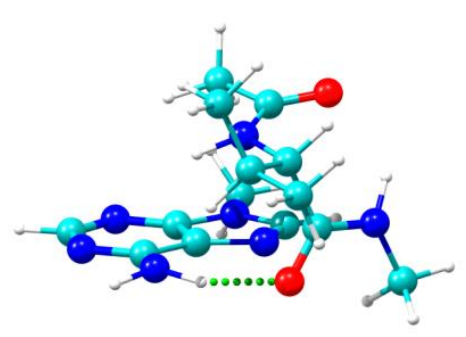

aVmA7

[7.95]

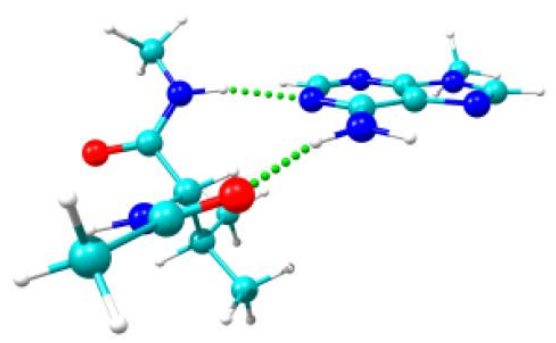

aVmA10

[9.61]

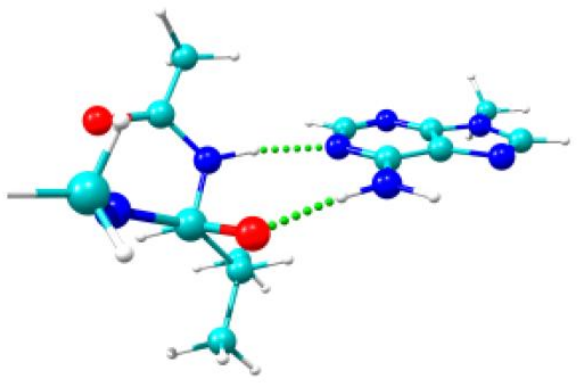

aVmA2

[2.49]

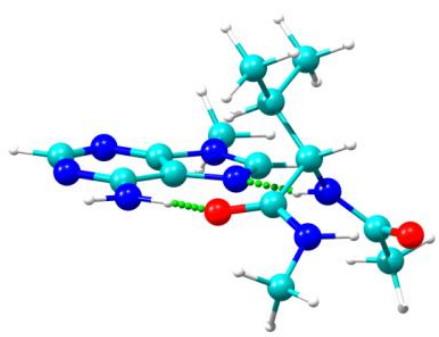

aVmA5

[7.88]

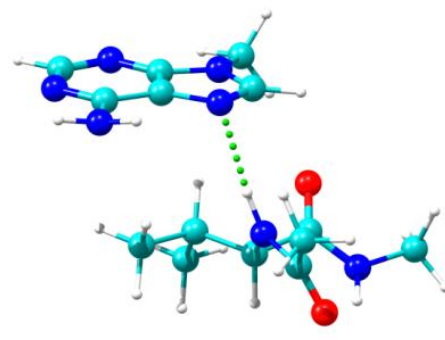

aVmA8

[9.24]

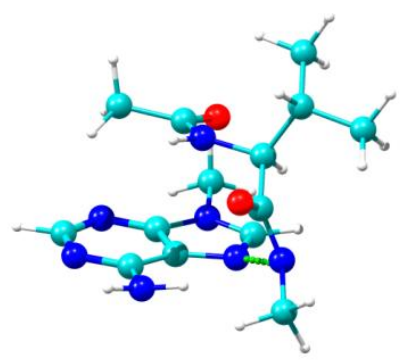

$a \bigvee m A 11$

[10.06]

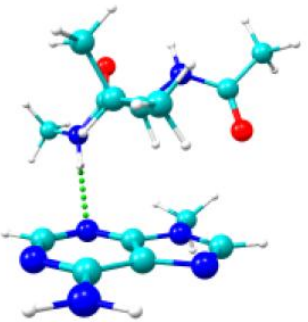

aVmA3

[7.00]

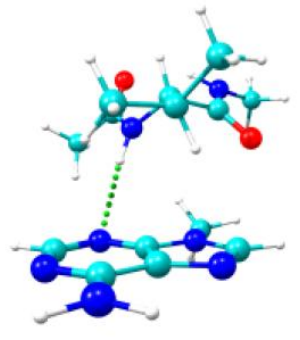

$\mathrm{aVmA6}$

[7.94]

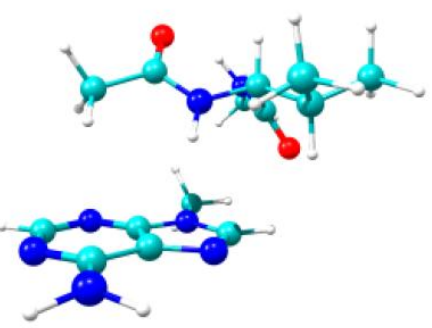

aVmA9

[9.48] 


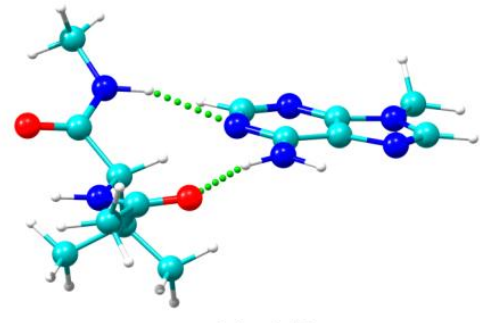

aVmA13

[11.22]

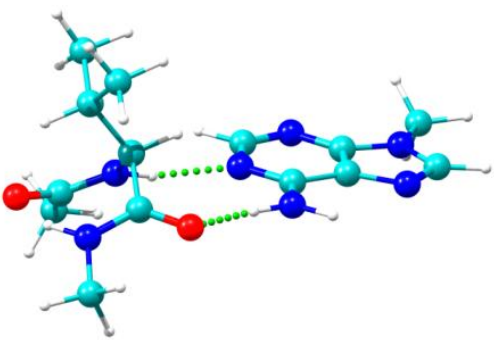

aVmA16

[12.57]

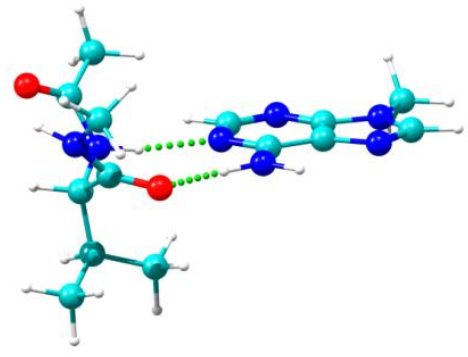

aVmA19

[15.27]

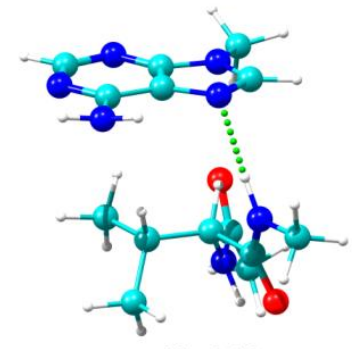

aVmA14

[11.43]

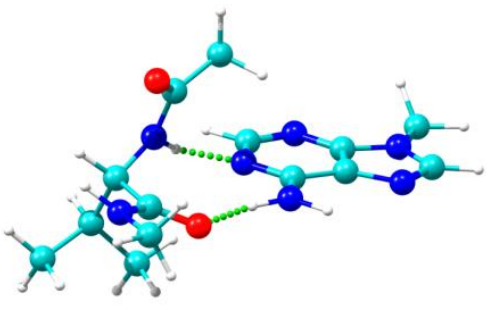

aVmA17

[13.29]

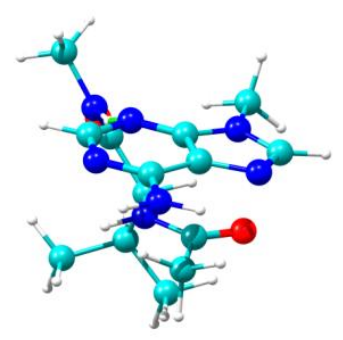

aVmA20

[15.31]

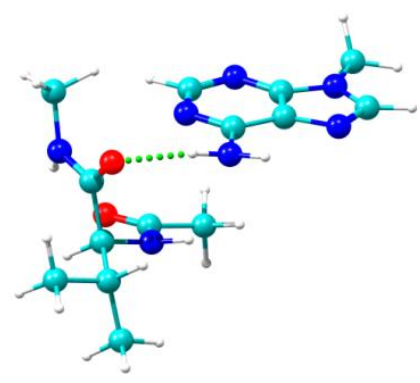

aVmA15

[11.67]

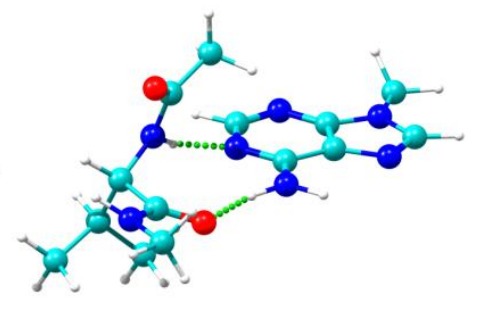

$a \mathrm{VmA} 18$

[13.40] 
Figure S15. The first 20 calculated structures for aImA at M06-2X/6-311++G(d,p) level together with their relative stability $\left(\mathrm{kJ} \mathrm{mol}^{-1}\right)$ in brackets. ZPE correction was applied to the energy values.
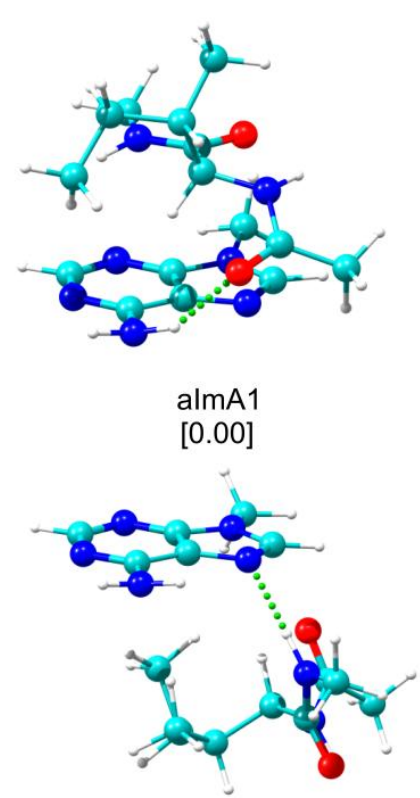

$\operatorname{almA4}$

[4.30]

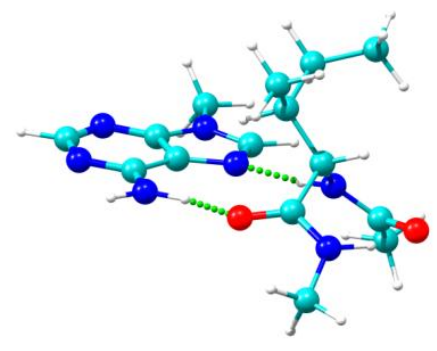

almA7

[7.76]

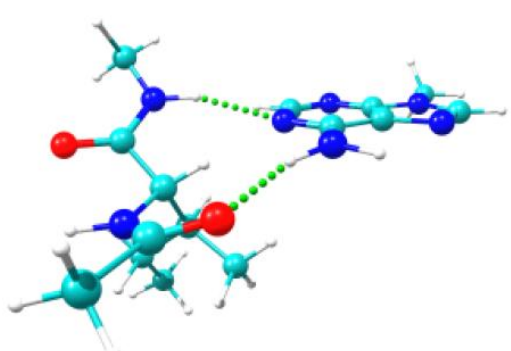

$\operatorname{almA10}$

[13.50]
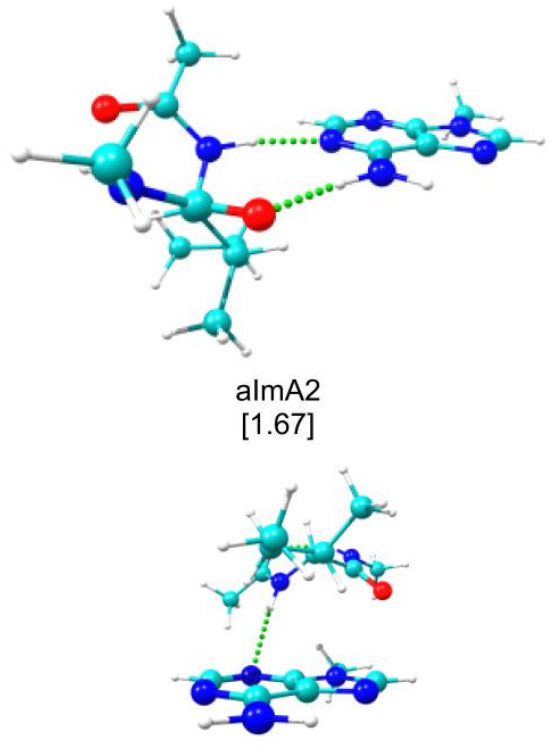

almA5

[5.60]

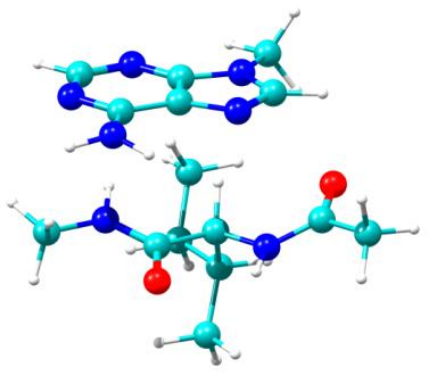

mA8

[8.01]

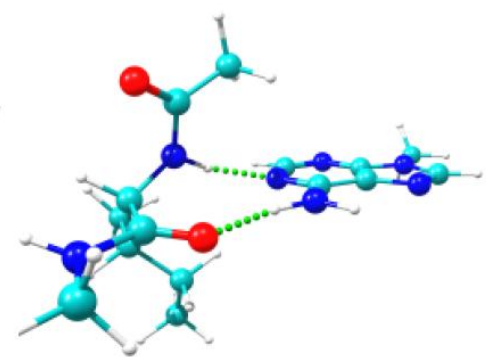

almA11

[13.64]

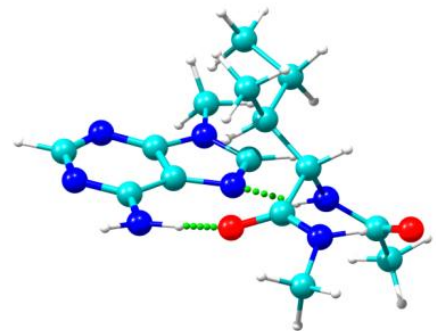

almA3

[4.30]

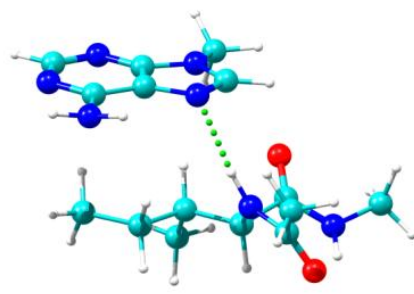

almA6

[5.89]

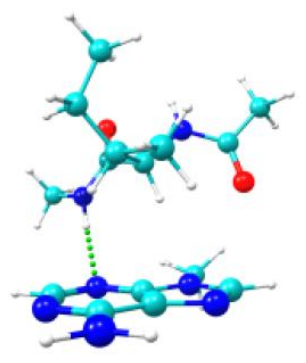

almA9

[11.16]

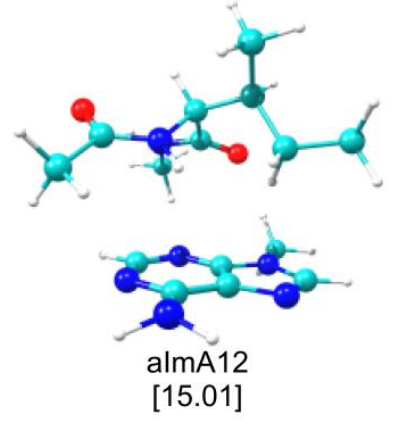




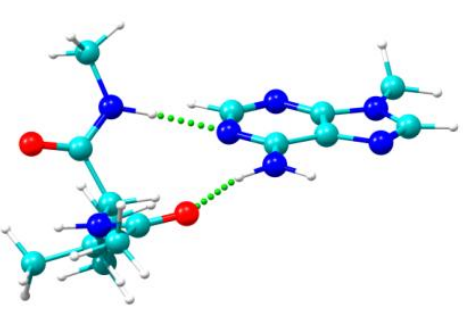

$\operatorname{almA13}$

[15.88]

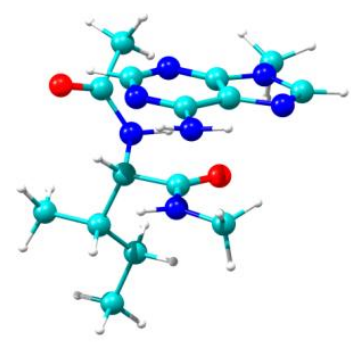

almA16

[17.77]

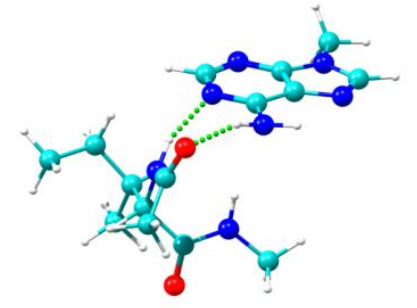

almA19

[20.92]

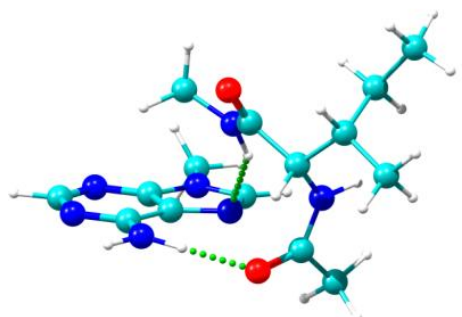

almA14

[16.05]

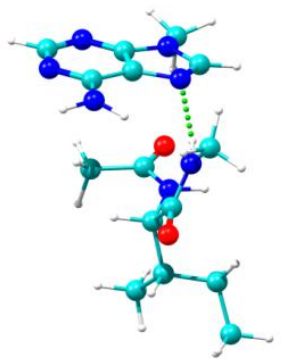

almA17

[18.69]

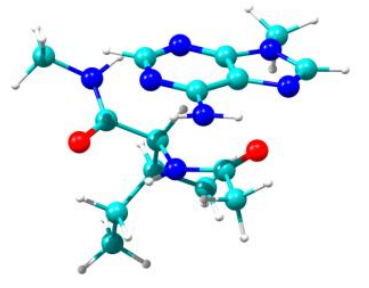

almA20

[21.20]

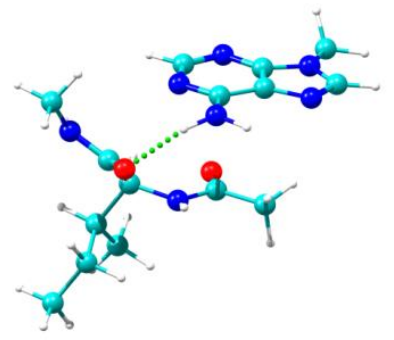

almA15

[17.50]

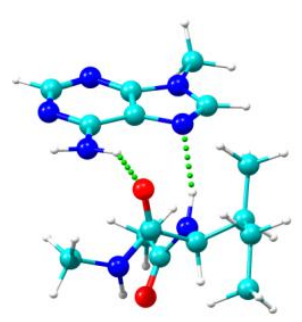

almA18

[20.04] 
Figure S16. The first 20 calculated structures for aNmA at M06-2X/6-311++G(d,p) level together with their relative stability $\left(\mathrm{kJ} \mathrm{mol}^{-1}\right)$ in brackets. ZPE correction was applied to the energy values.

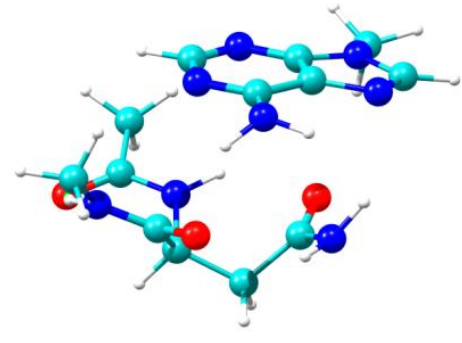

aNmA1

[0.00]

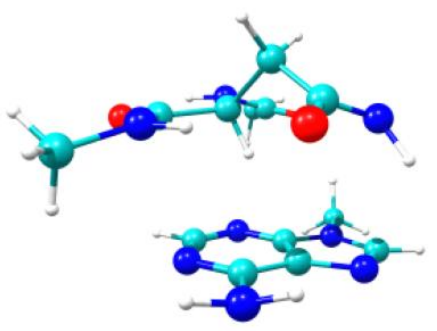

aNmA4

[7.91]

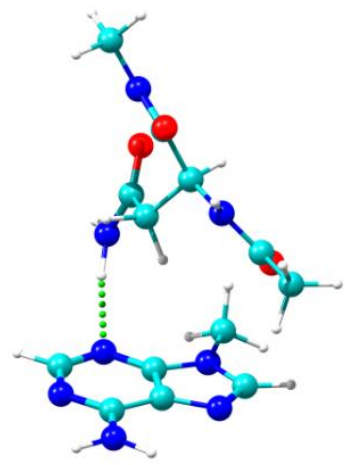

aNmA7

[9.16]

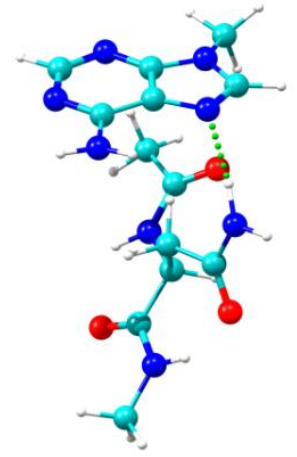

aNmA10

[10.93]

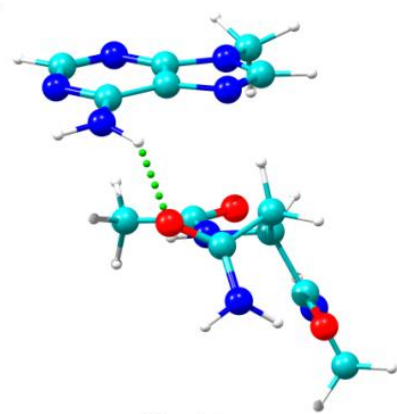

aNmA2

[6.71]

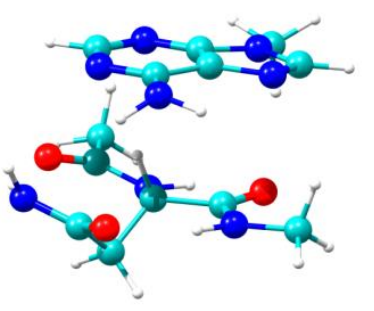

aNmA5

[8.29]

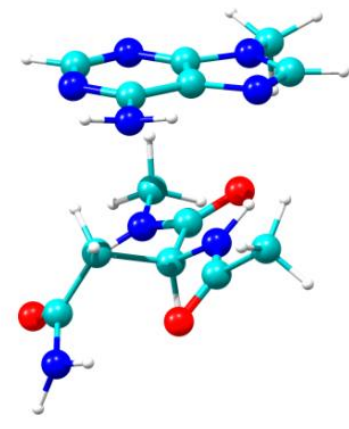

aNmA8

[9.89]

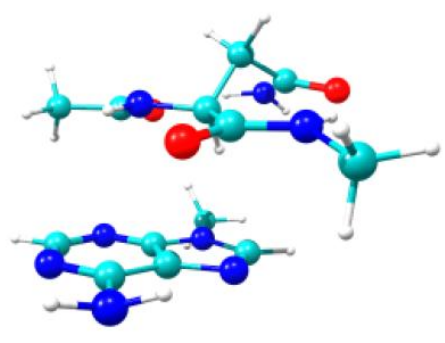

aNmA11

[12.41]

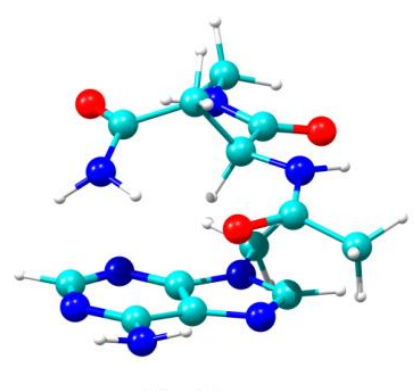

aNmA3

[7.32]

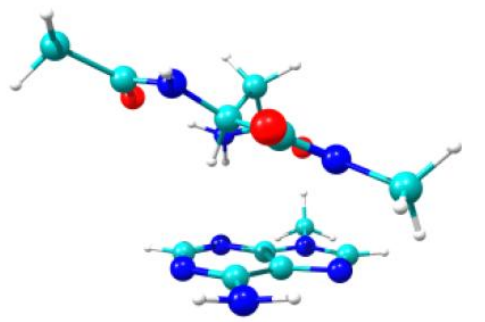

aNmA6

[8.92]

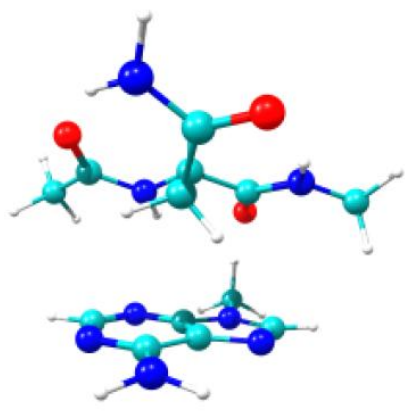

aNmA9

[10.82]

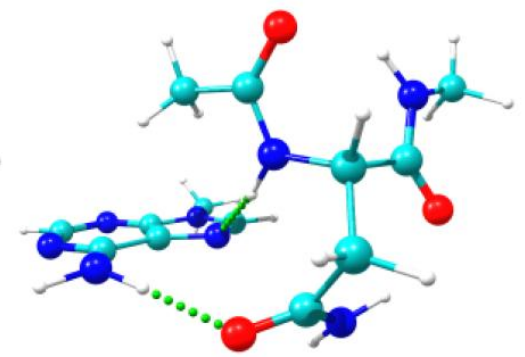

aNmA12

[14.23] 


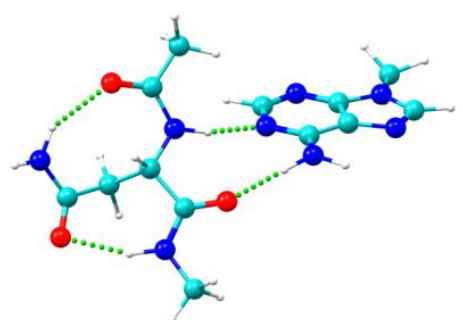

aNmA13

[16.12]

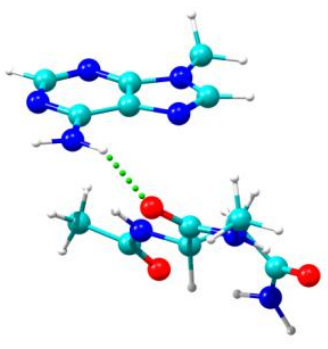

aNmA16

[18.11]

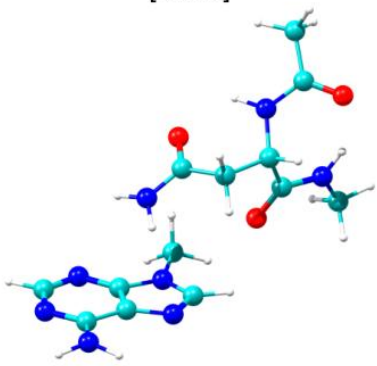

aNmA19

[22.77]

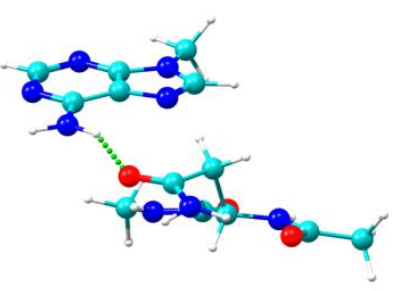

aNmA14

[17.16]

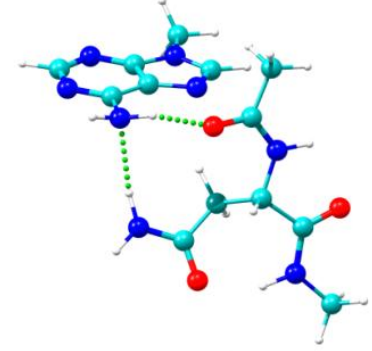

aNmA17

[18.83]

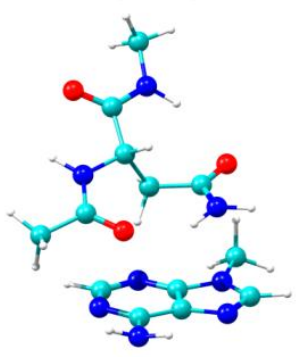

aNmA20

[24.81]

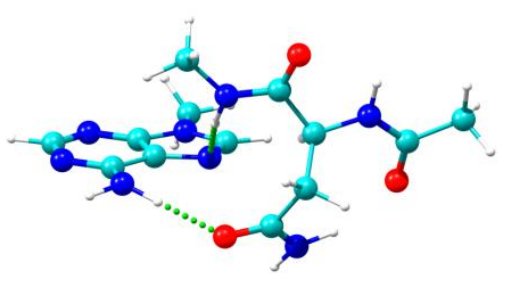

aNmA15

[17.97]

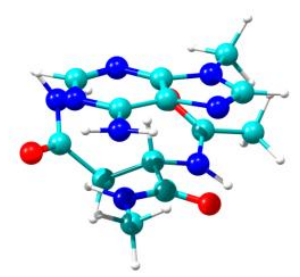

aNmA18

[19.53] 
Figure S17. The first 20 calculated structures for $a Q m A$ at $M 06-2 X / 6-311++G(d, p)$ level together with their relative stability $\left(\mathrm{kJ} \mathrm{mol}^{-1}\right)$ in brackets. ZPE correction was applied to the energy values.

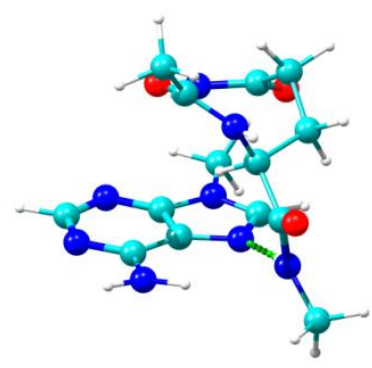

aQmA1

[0.00]

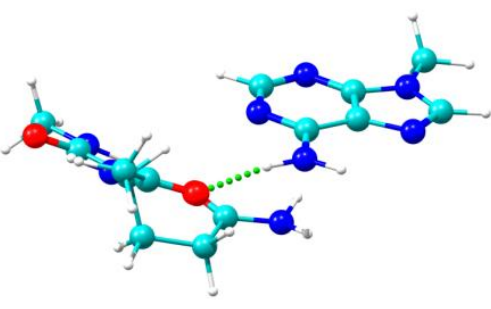

aQmA4

[17.08]

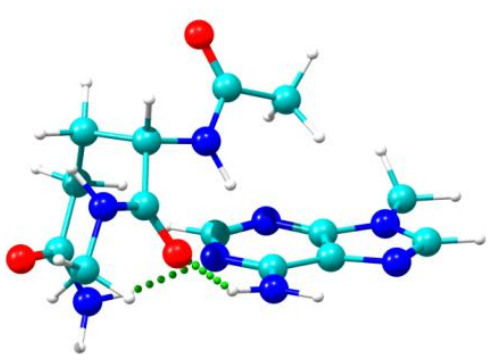

aQmA7

[20.30]

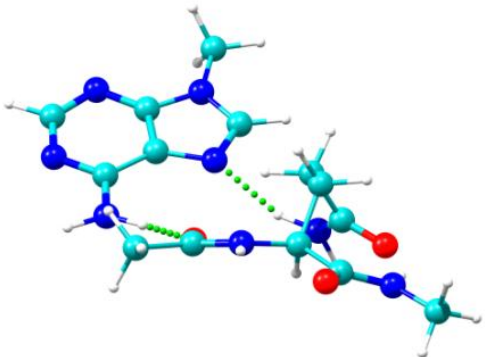

aQmA10

[21.63]

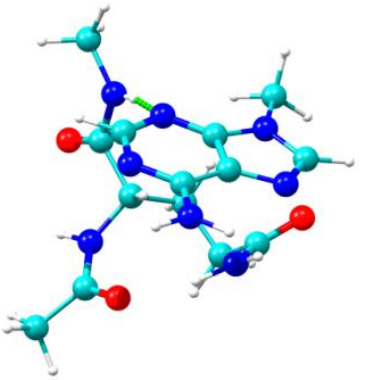

aQmA2

[7.28]

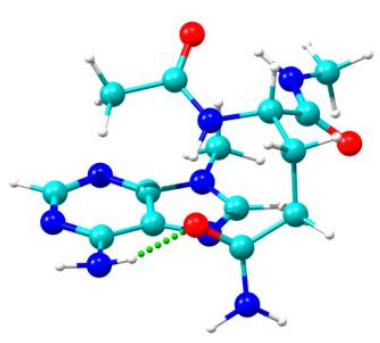

aQmA5

[17.22]

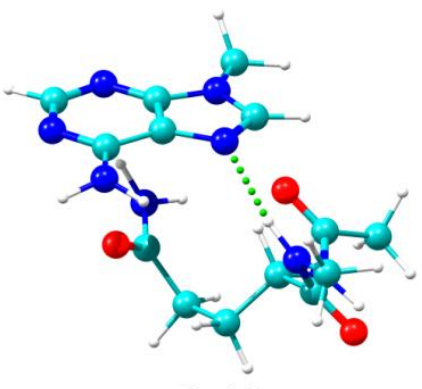

aQmA8

[20.53]

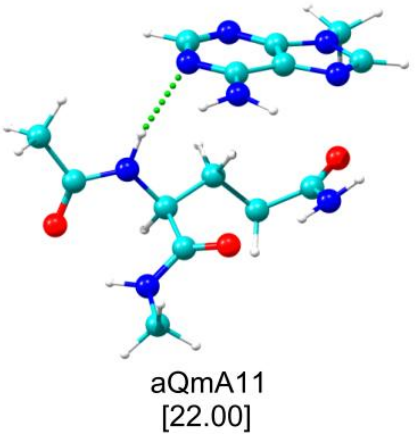

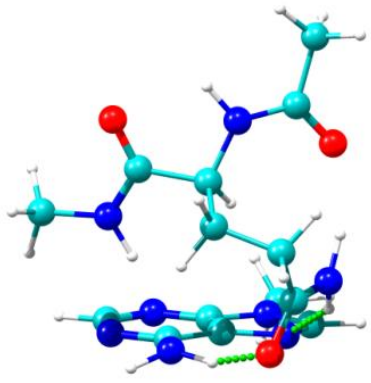

$\mathrm{aQmA3}$

[14.26]

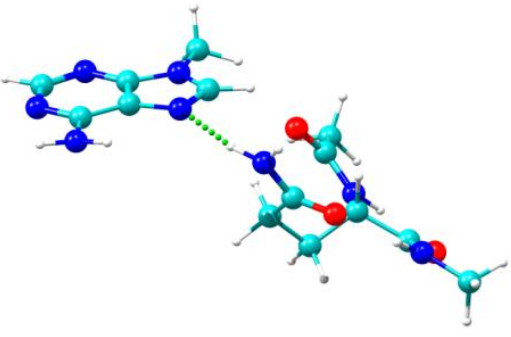

aQmA6

[18.22]

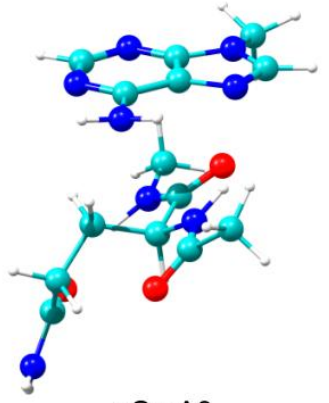

QmA9

[20.69]

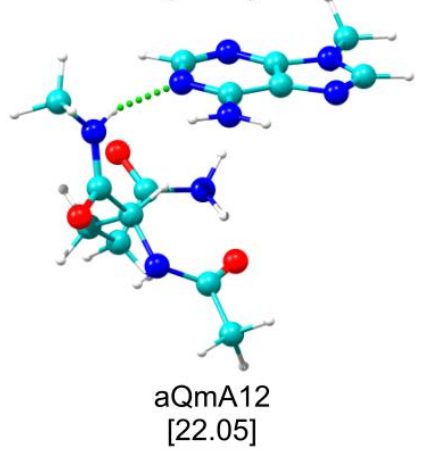




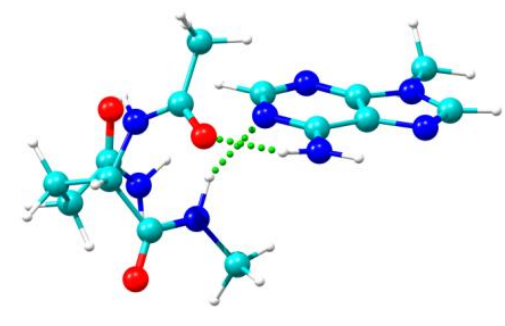

aQmA13

[22.13]

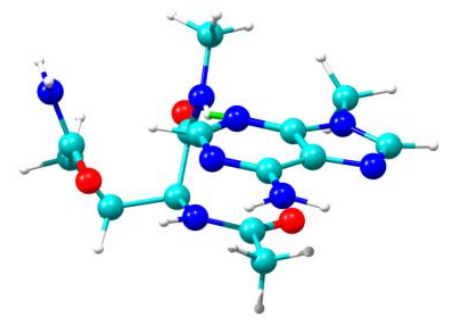

aQmA16

[23.17]

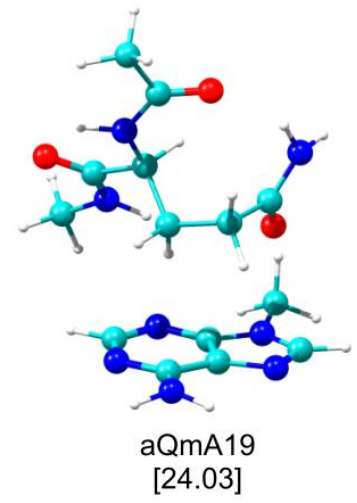

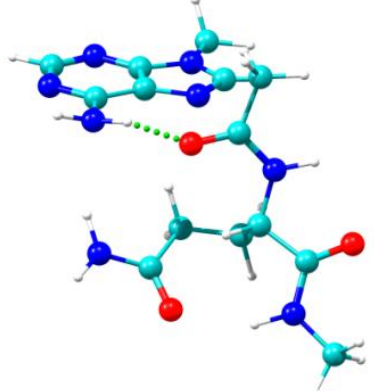

aQmA14

[22.69]

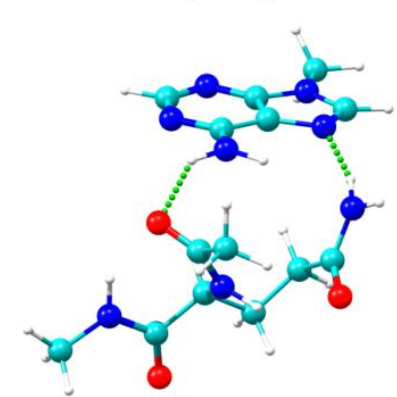

aQmA17

[23.52]

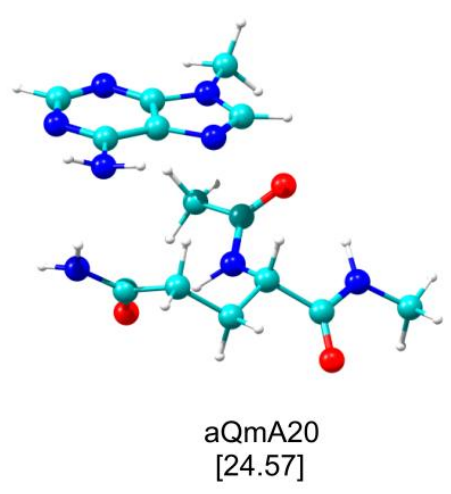

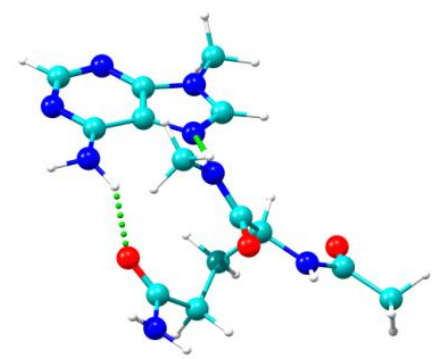

aQmA15

[22.77]

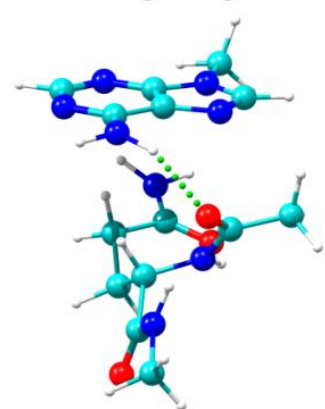

aQmA18

[23.92] 
Figure S18. The first 20 calculated structures for aRmA at M06-2X/6-311++G(d,p) level together with their relative stability $\left(\mathrm{kJ} \mathrm{mol}^{-1}\right)$ in brackets. ZPE correction was applied to the energy values.

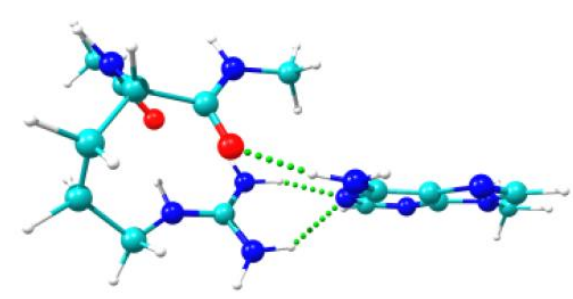

aRmA1

[0.00]
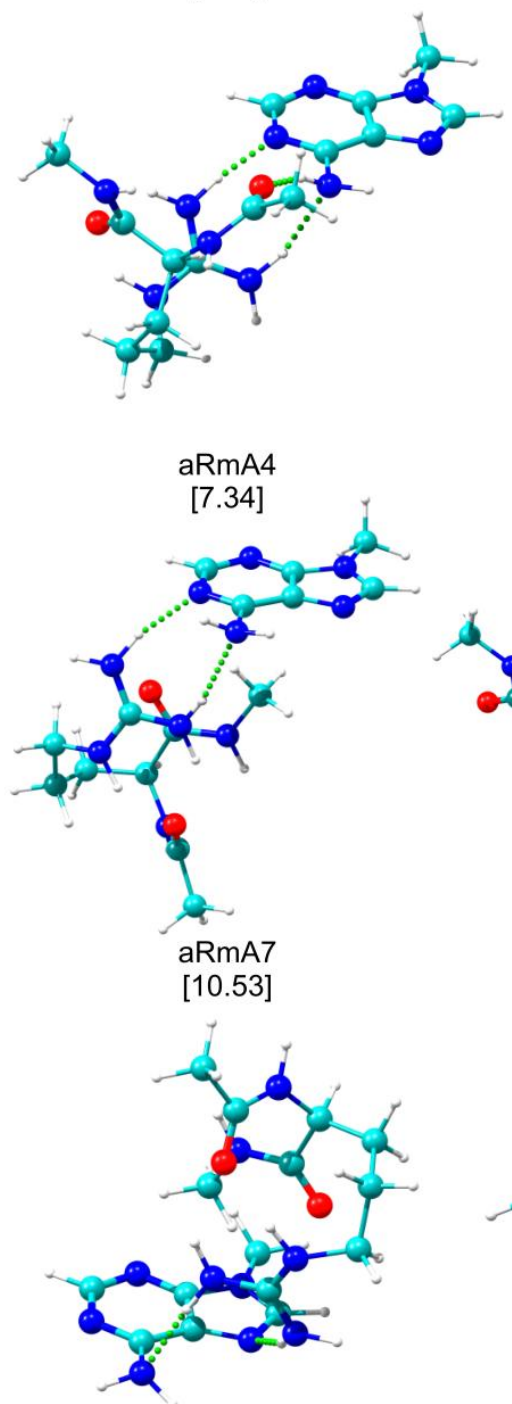

aRmA10

[12.46]

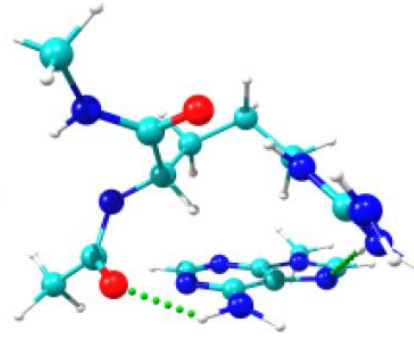

aRmA2

[1.42]

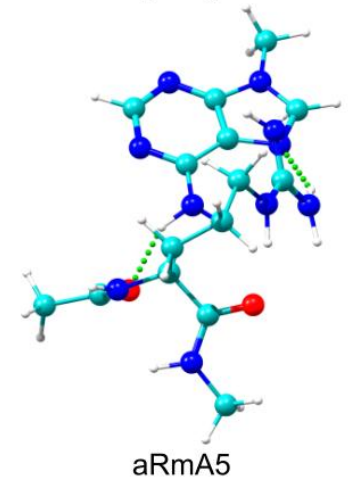

[8.66]
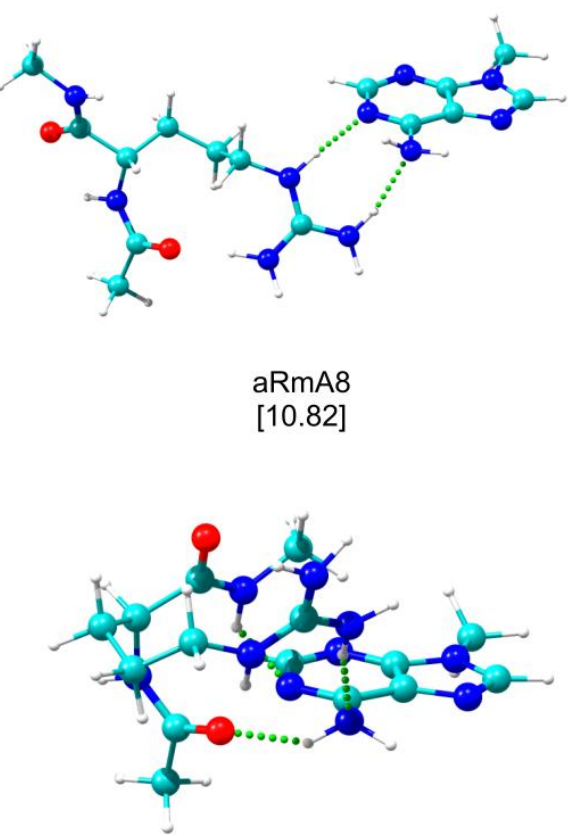

aRmA11

[13.39]
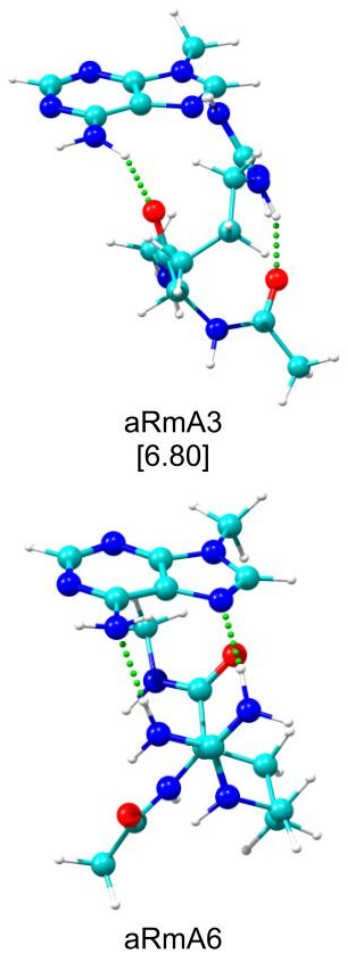

[9.11]

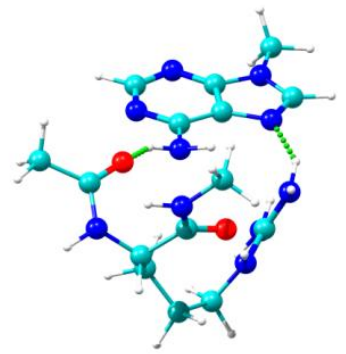

aRmA9

[11.92]

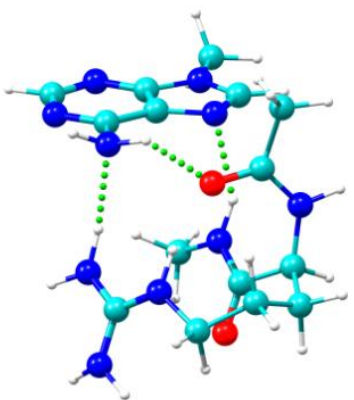

aRmA12

[13.92] 


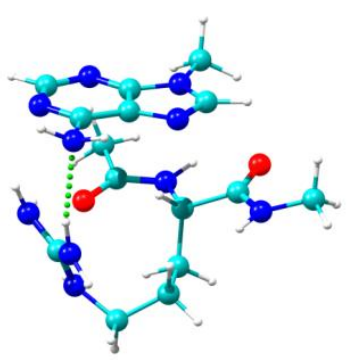

aRmA13

[14.16]

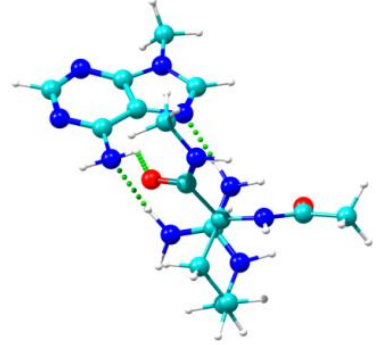

aRmA16

[17.39]

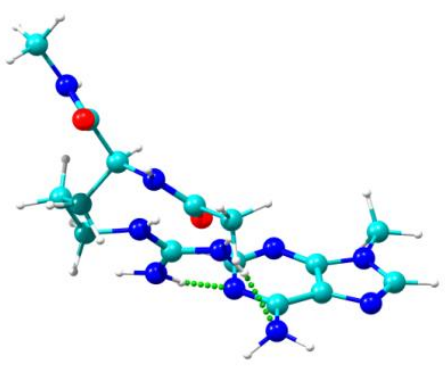

aRmA14

[15.88]

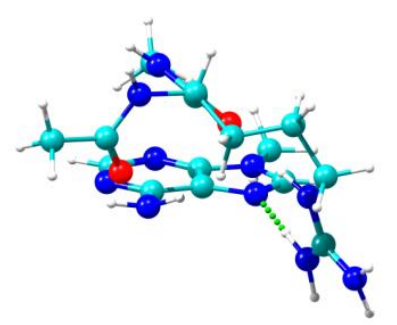

aRmA17

[17.64]

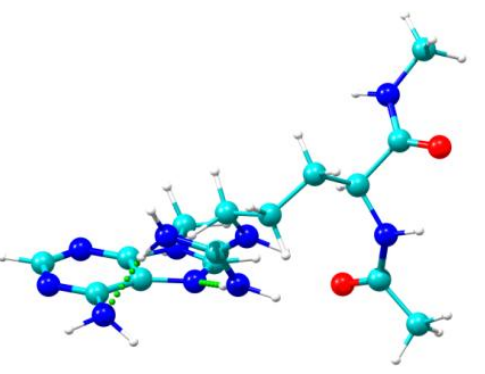

aRmA15

[17.29]

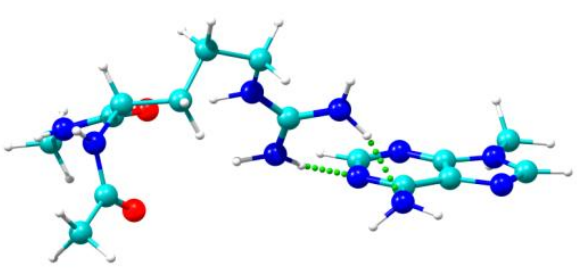

aRmA19

[18.79]

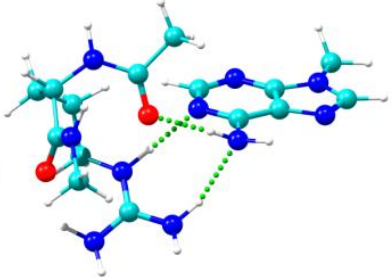

aRmA20

[19.09]

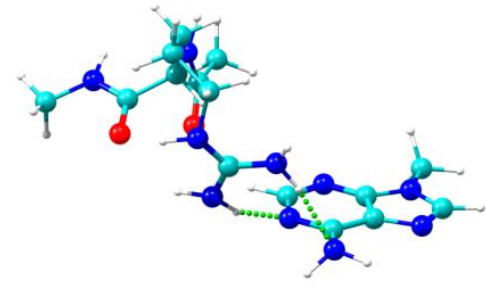

RmA18

[18.70] 
Figure S19. The first 20 calculated structures for $a A m G$ at $M 06-2 X / 6-311++G(d, p)$ level together with their relative stability $\left(\mathrm{kJ} \mathrm{mol}^{-1}\right)$ in brackets. ZPE correction was applied to the energy values.

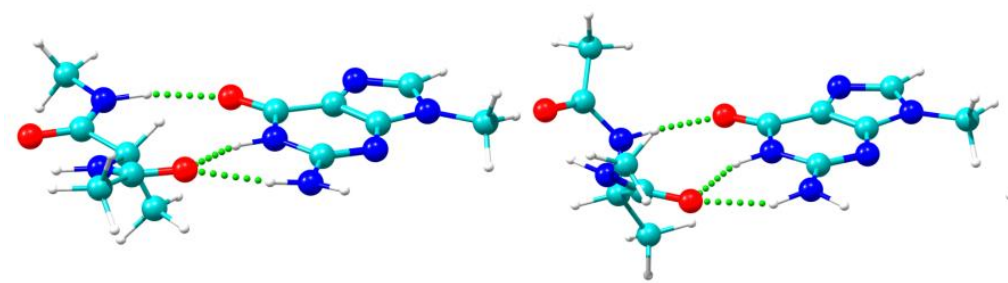

aAmG1

[0.00]

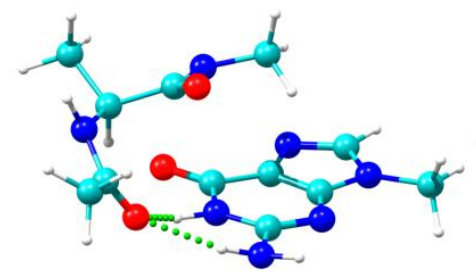

aAmG4

[12.72]

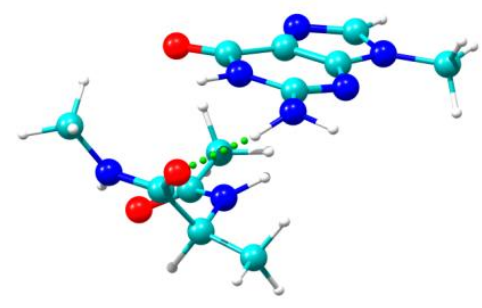

aAmG7

[15.34]

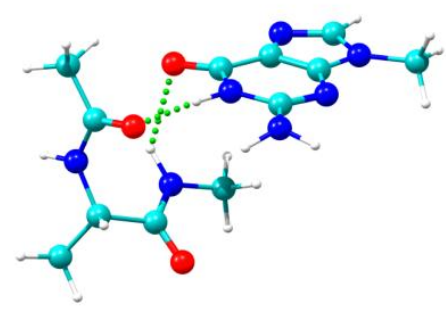

aAmG10

[19.36]

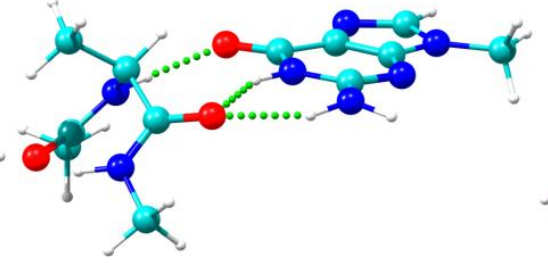

aAmG5

[12.89]

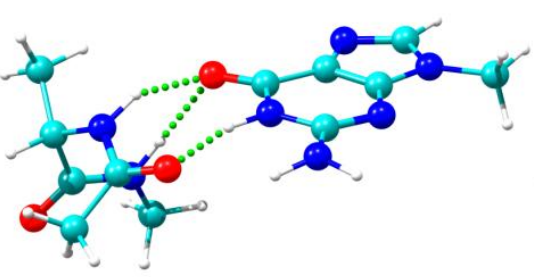

aAmG8

[16.78]

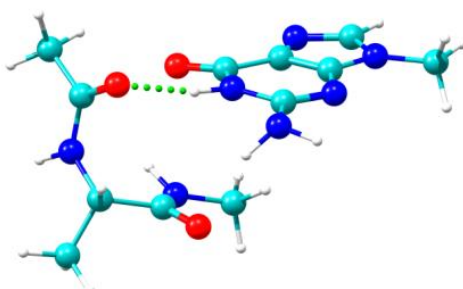

aAmG11

[20.75]

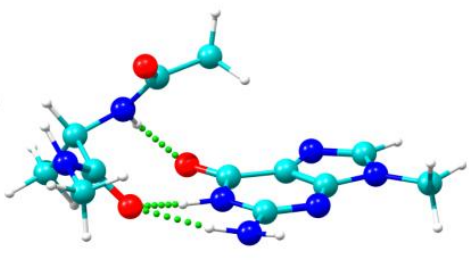

aAmG3

[6.97]

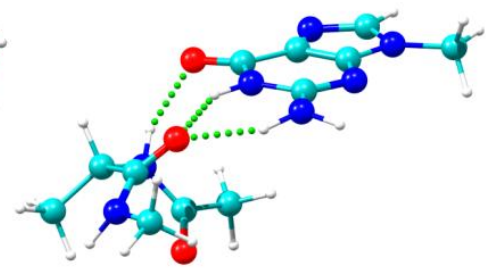

aAmG6

[14.95]

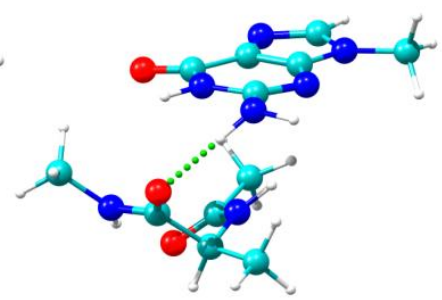

aAmG9

[17.11]

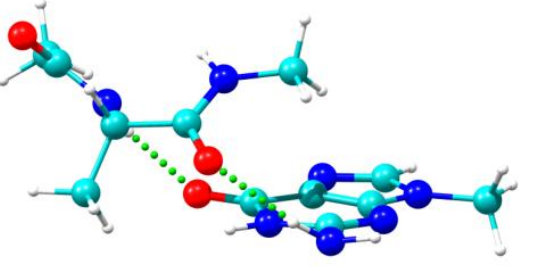

aAmG12

[21.88] 


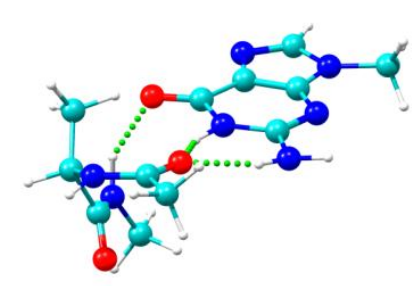

aAmG13

[22.20]

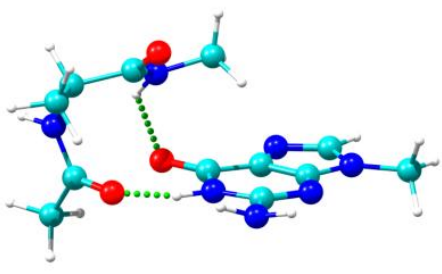

aAmG16

[24.51]

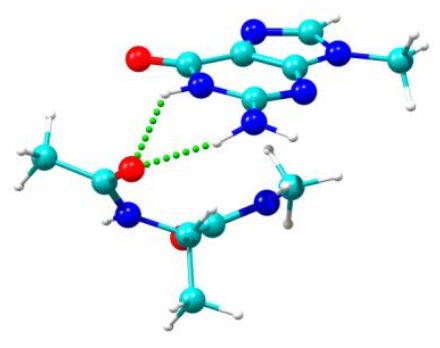

aAmG19

[27.70]

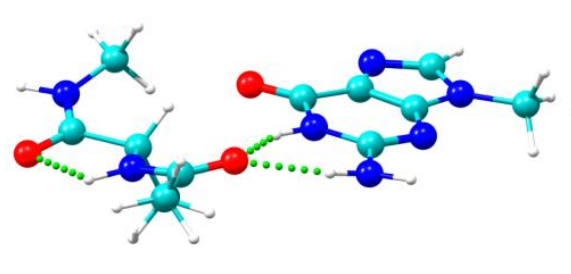

aAmG14

[23.35]

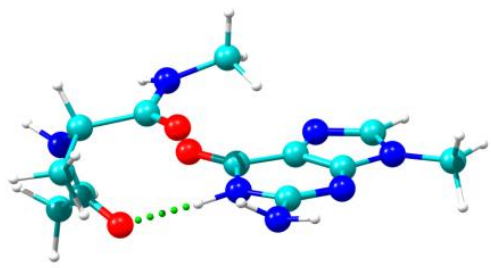

aAmG17

[25.94]
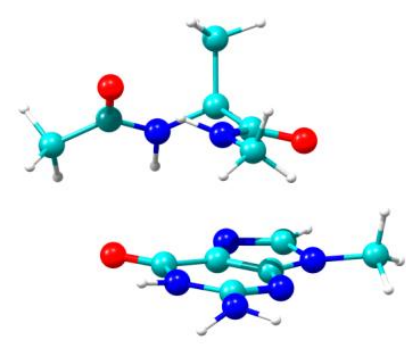

aAmG20

[29.55]

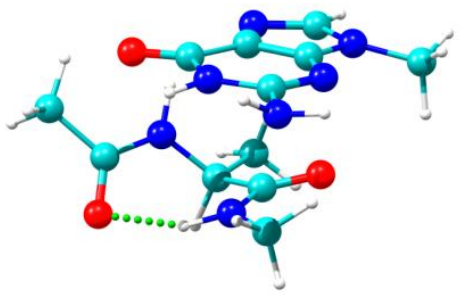

aAmG15

[24.37]

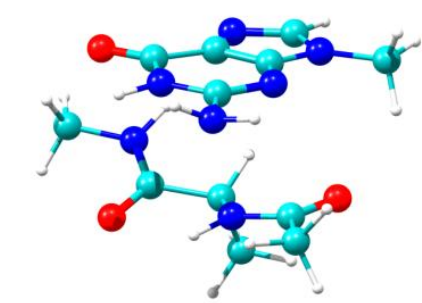

aAmG18

[26.58] 
Figure S20. The first 20 calculated structures for $\mathrm{aVmG}$ at $\mathrm{M} 06-2 \mathrm{X} / 6-311++\mathrm{G}(\mathrm{d}, \mathrm{p})$ level together with their relative stability $\left(\mathrm{kJ} \mathrm{mol}^{-1}\right)$ in brackets. ZPE correction was applied to the energy values.

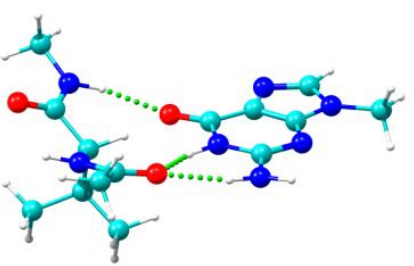

$a \vee m G 1$

[0.00]

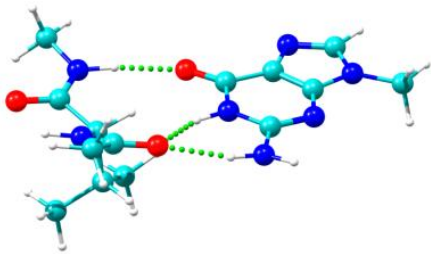

$\mathrm{aVmG4}$

[2.46]

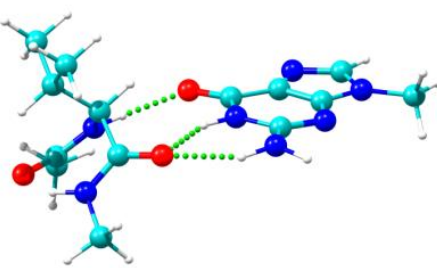

aVmG7

[8.92]

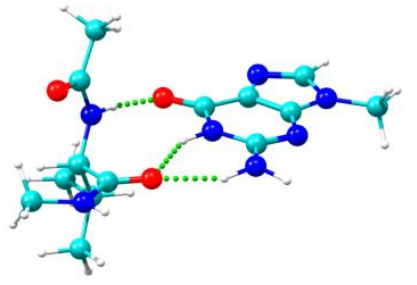

$\mathrm{aVmG10}$

[12.34]

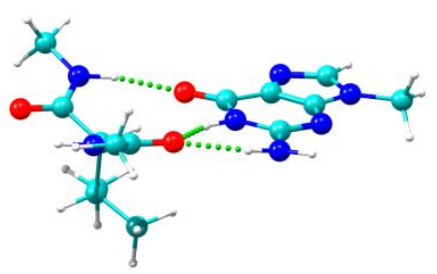

$\mathrm{aVmG2}$

[0.01]

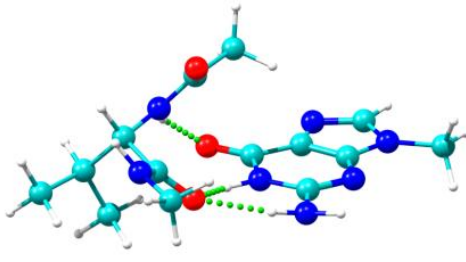

aVmG5

[7.91]

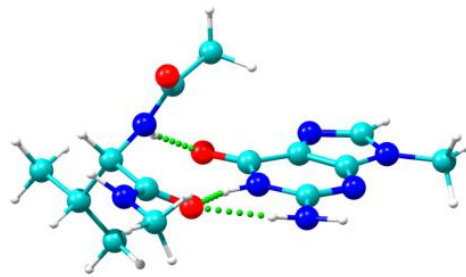

aVmG8

[9.74]

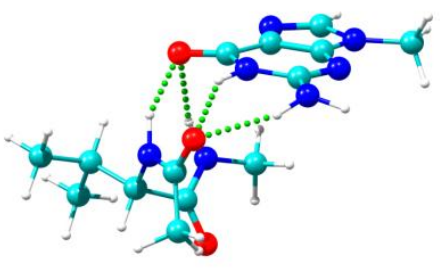

$\mathrm{aVmG} 11$

[16.12]

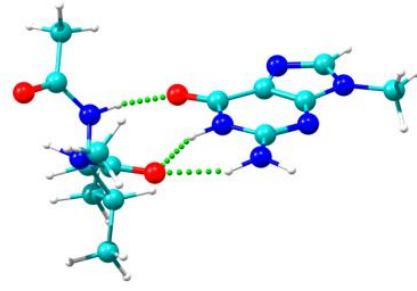

aVmG3

[0.22]

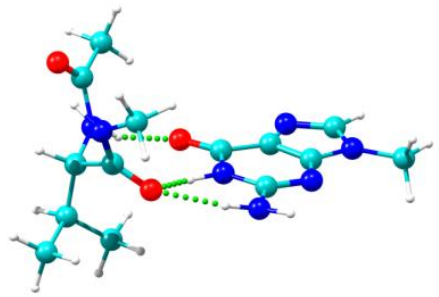

aVmG6

[8.38]

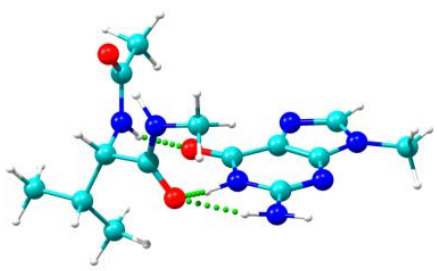

aVmG9

[11.89]

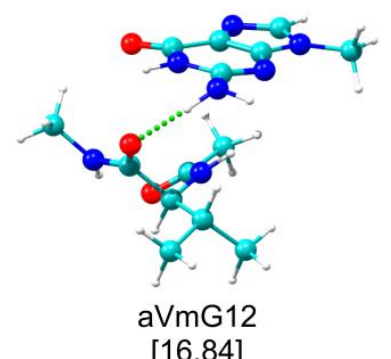




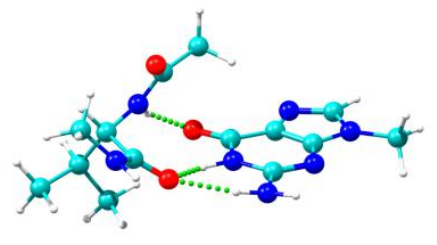

aVmG13

[16.93]

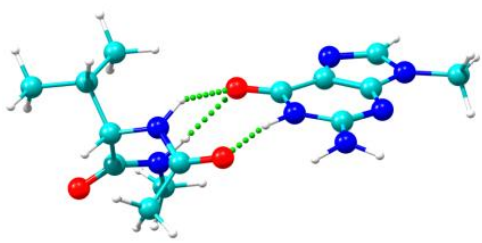

aVmG16

[19.34]

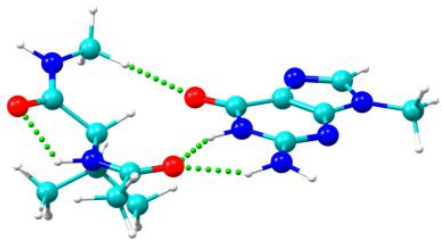

aVmG19

[21.70]

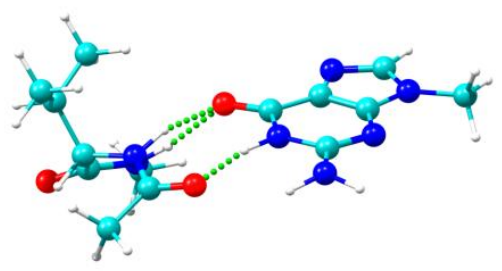

$\mathrm{aVmG} 14$

[17.21]

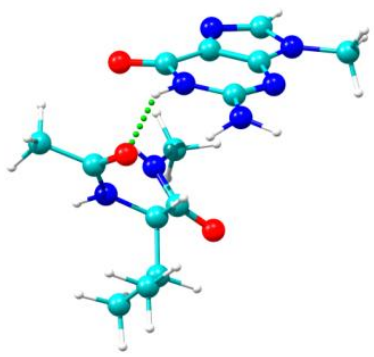

aVmG17

[19.96]

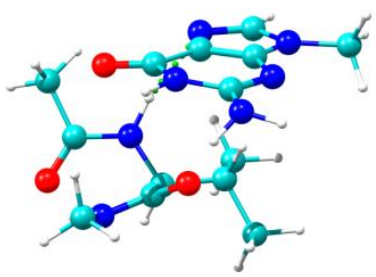

aVmG20

[24.69]

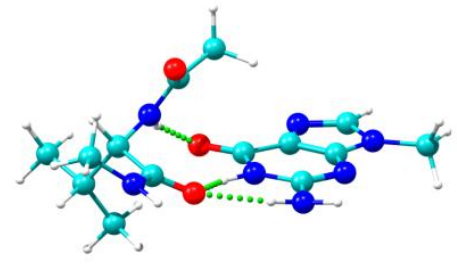

$\mathrm{aVmG15}$

[17.45]

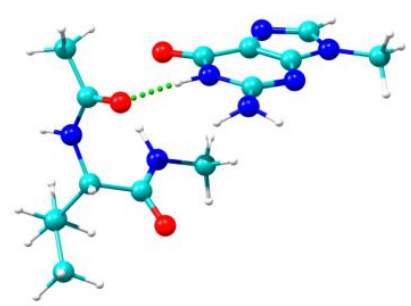

aVmG18

[21.40] 
Figure S21. The first 20 calculated structures for aImG at M06-2X/6-311++G(d,p) level together with their relative stability $\left(\mathrm{kJ} \mathrm{mol}^{-1}\right)$ in brackets. ZPE correction was applied to the energy values.

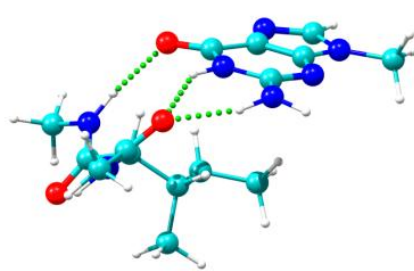

almG1

$[0.00]$

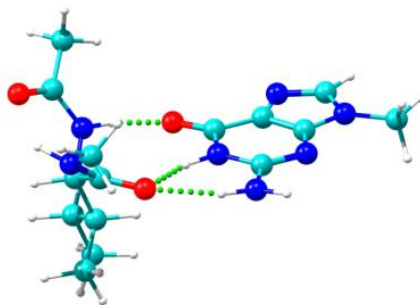

almG4

[1.42]

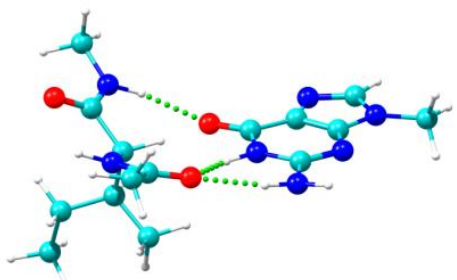

almG7

[3.18]

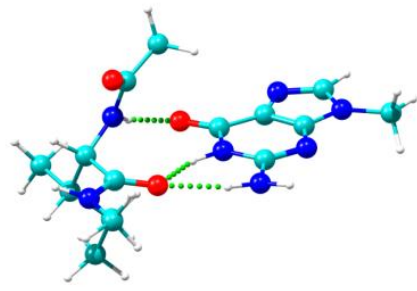

almG10

[10.66]

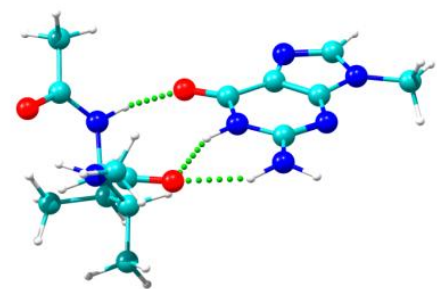

almG2

[0.72]

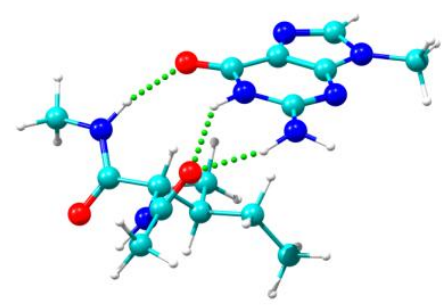

almG5

[2.31]

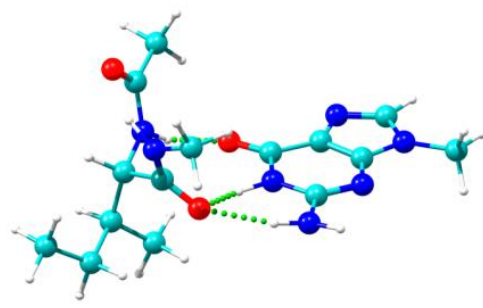

almG8

[9.23]

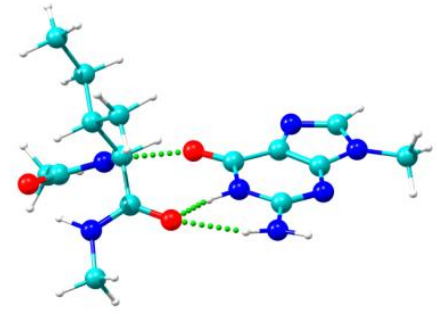

almG11

[12.18]

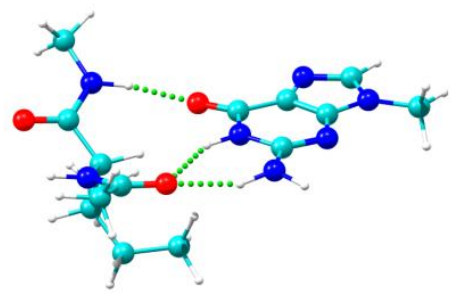

almG3

[0.77]

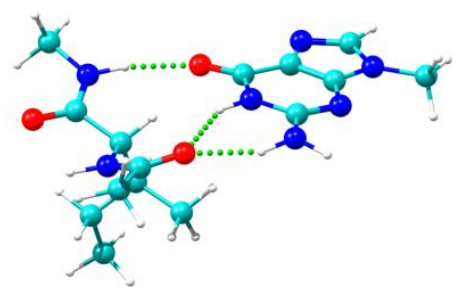

almG6

[2.34]

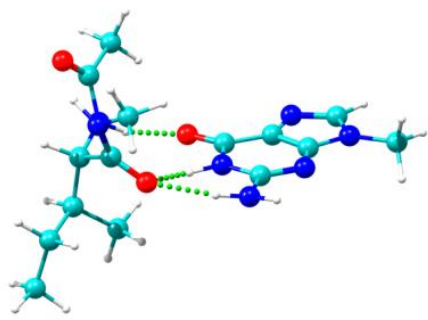

almG9

[10.39]

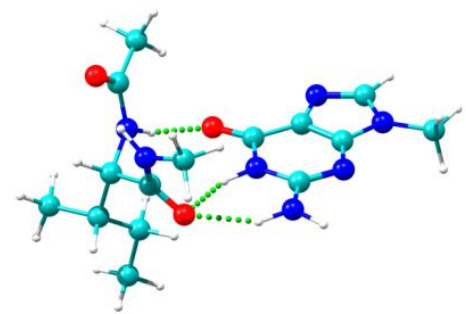

almG12

[15.31] 


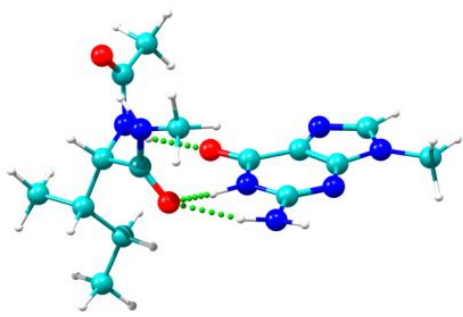

almG13

[15.65]

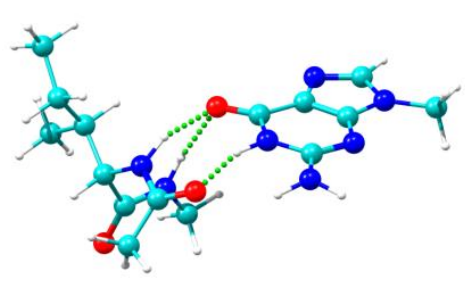

almG16

[17.76]

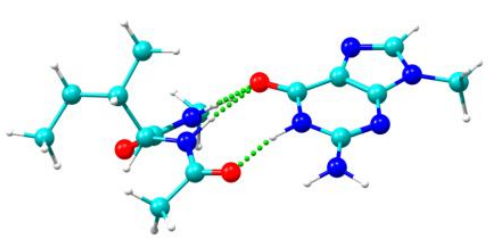

almG19

[20.27]

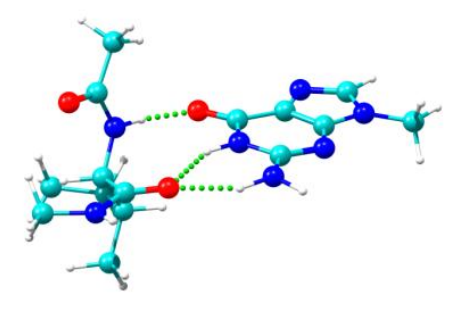

almG14

[15.81]

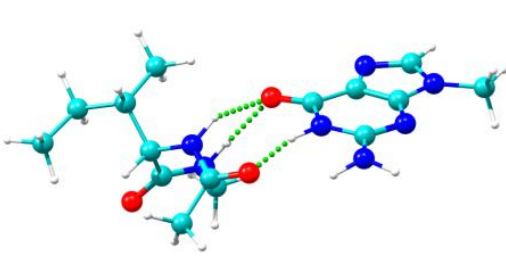

almG17

[19.17]

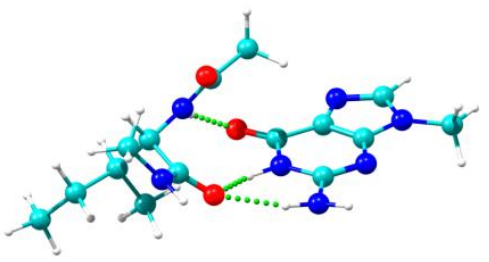

almG20

[20.40]

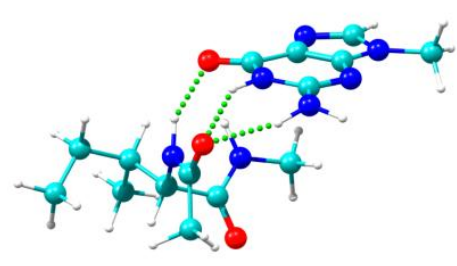

almG15

[17.05]

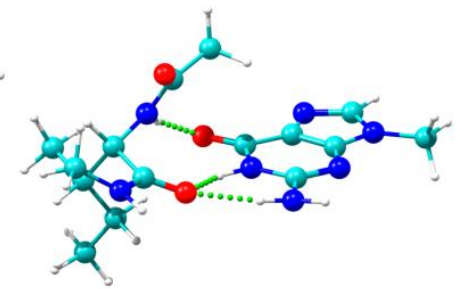

almG18

[19.74] 
Figure S22. The first 20 calculated structures for $\mathrm{aNmG}$ at $\mathrm{M} 06-2 \mathrm{X} / 6-311++\mathrm{G}(\mathrm{d}, \mathrm{p})$ level together with their relative stability $\left(\mathrm{kJ} \mathrm{mol}^{-1}\right)$ in brackets. ZPE correction was applied to the energy values.

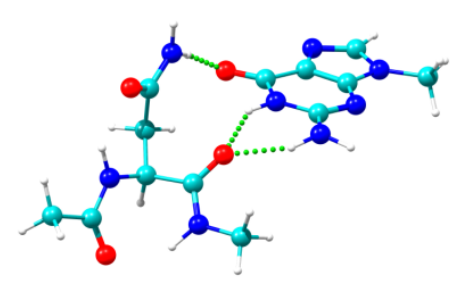

aNmG1

[0.00]

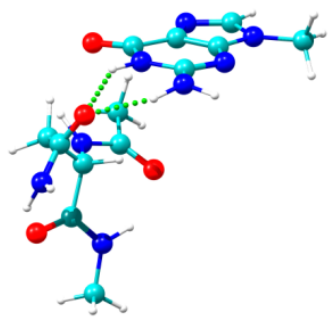

aNmG4

[8.98]

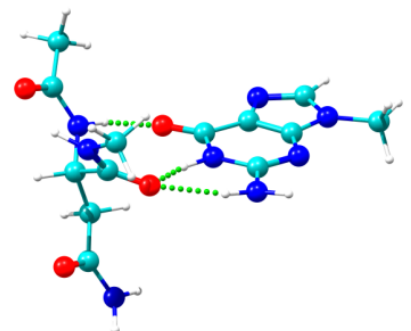

aNmG7

[12.15]

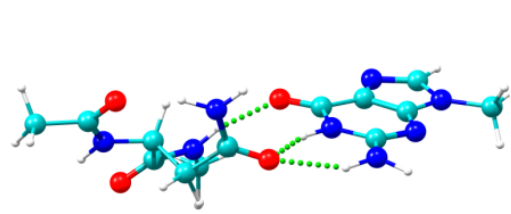

aNmG10

[14.01]

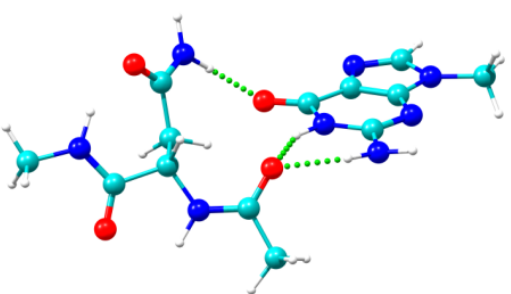

aNmG2

[0.93]

aNmG3

[6.41]

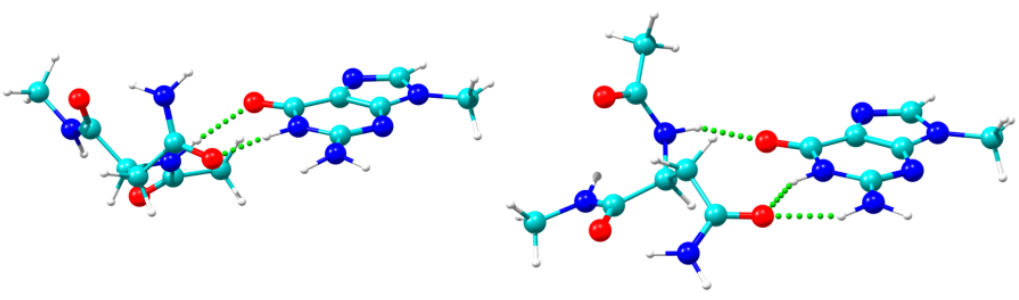

aNmG5

[10.09]

[12.03]

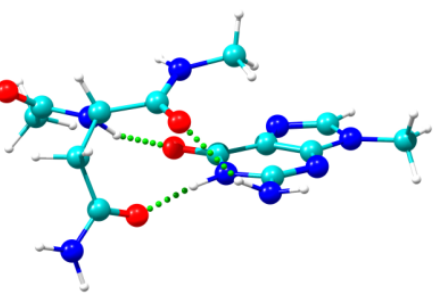

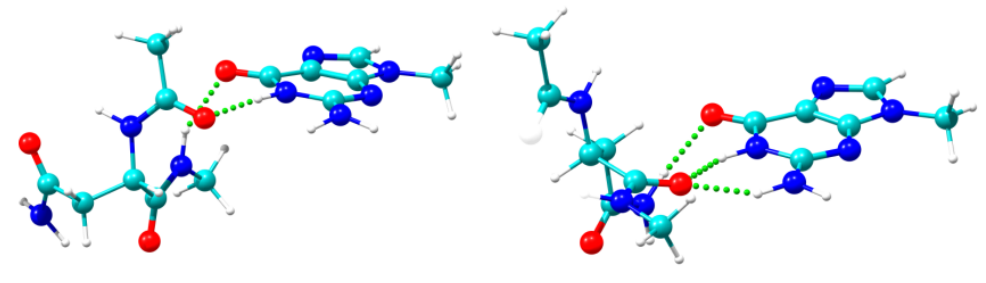

aNmG8

[13.04]

aNmG9

[13.62]
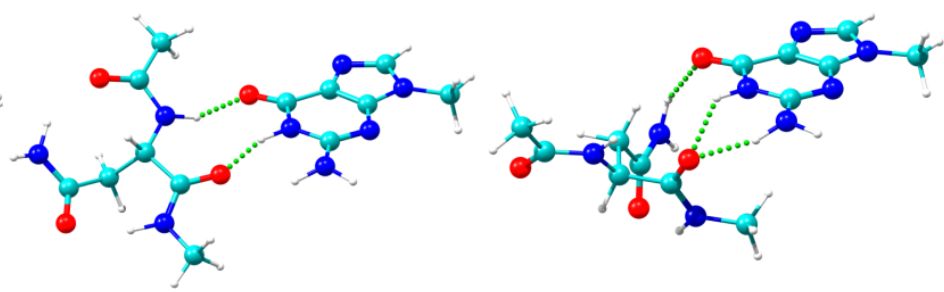

aNmG11

[14.46]

aNmG12

[16.44] 


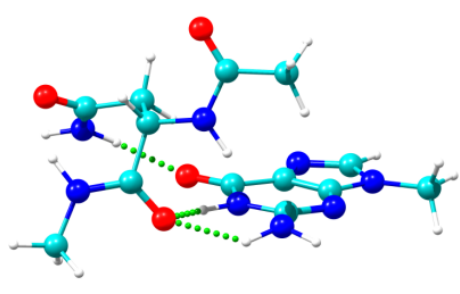

aNmG13

[16.65]

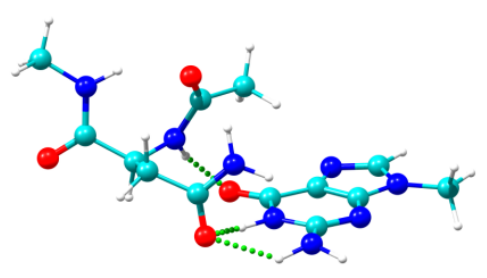

aNmG14

[16.86]

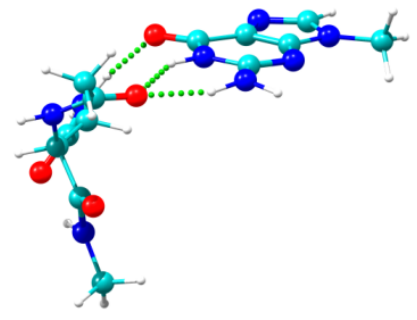

aNmG15

[17.45]

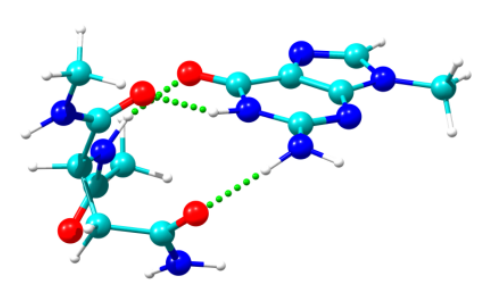

aNmG16

[19.91]

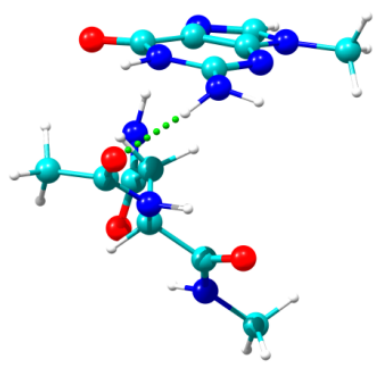

aNmG19

[22.70]

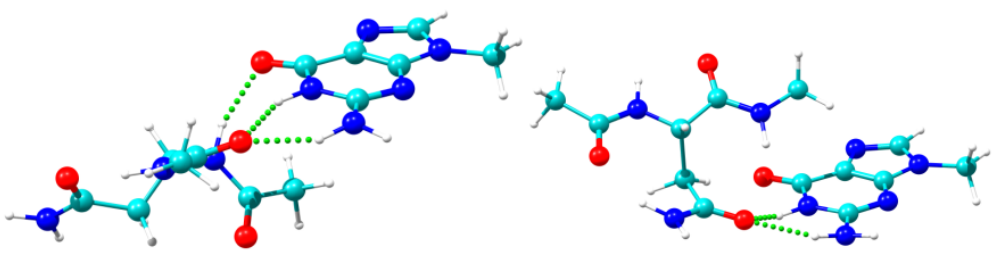

aNmG17

[19.98]

aNmG18

[20.35]

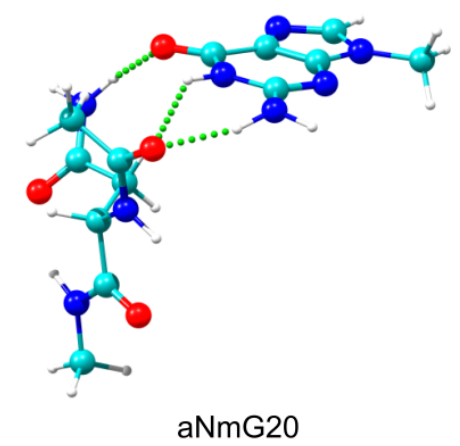

[23.11] 
Figure S23. The first 20 calculated structures for $a Q m G$ at $M 06-2 X / 6-311++G(d, p)$ level together with their relative stability $\left(\mathrm{kJ} \mathrm{mol}^{-1}\right)$ in brackets. ZPE correction was applied to the energy values.

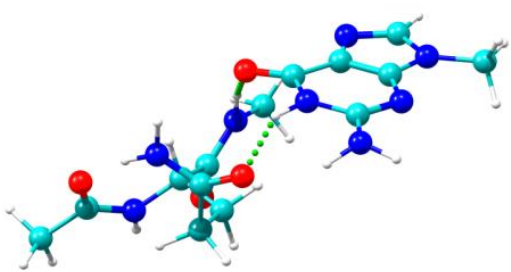

$\mathrm{aQmG1}$

[0.00]

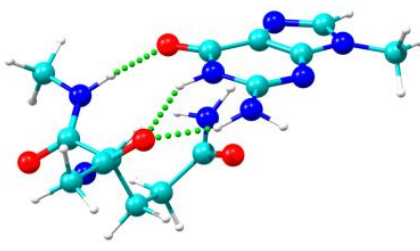

$\mathrm{aQmG2}$

[0.54]

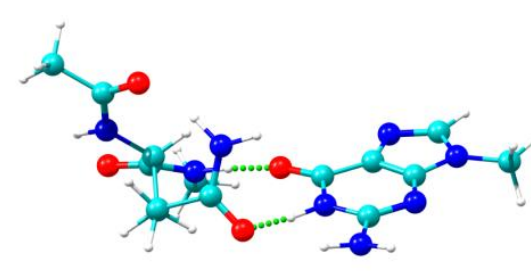

aQmG4

[2.30]

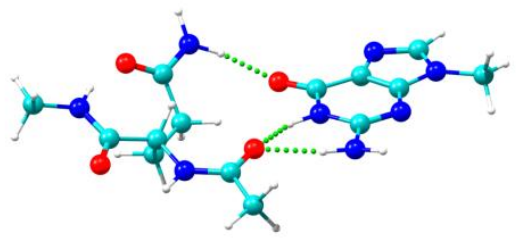

aQmG7

[8.75]

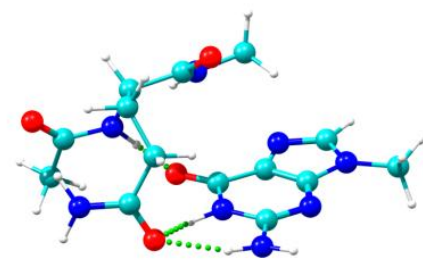

$\mathrm{aQmG10}$

[12.55]

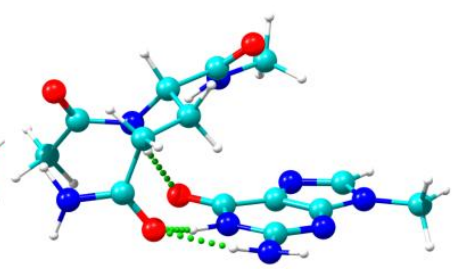

$\mathrm{aQmG5}$

[5.85]

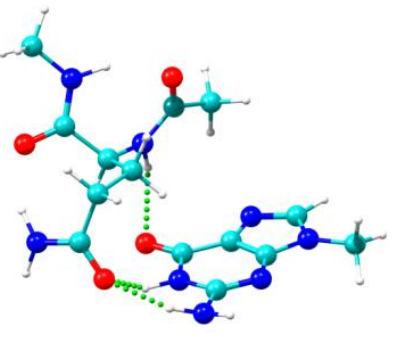

aQmG8

[9.81]

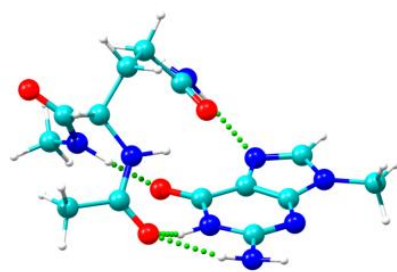

aQmG3

[1.68]

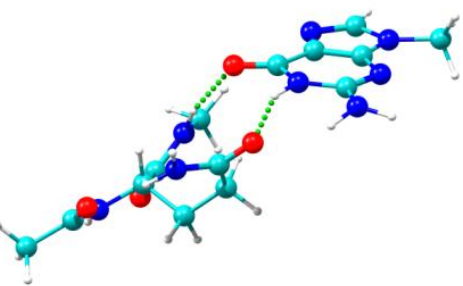

aQmG6

[8.59]

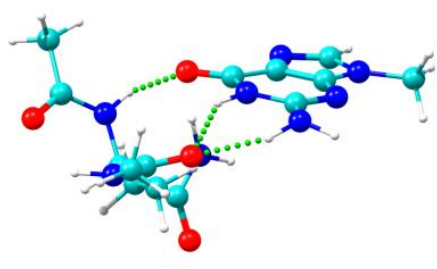

aQmG9

[12.08] 


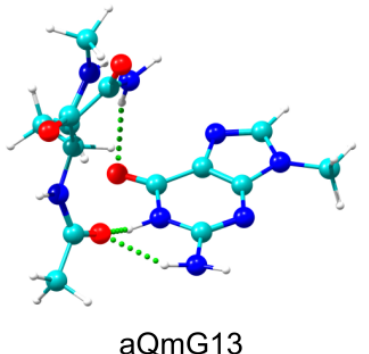

[13.11]

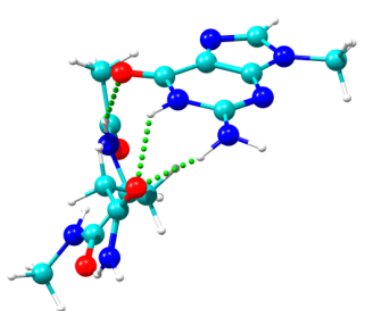

aQmG16

[15.90]

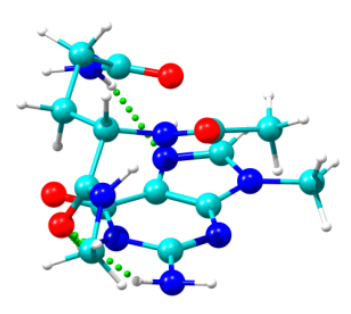

aQmG19

[17.47]

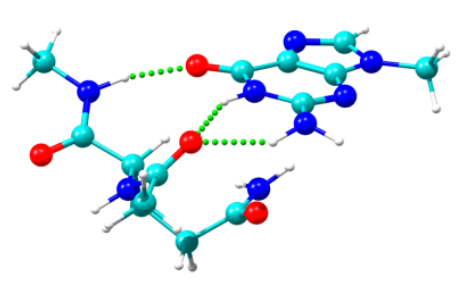

$\mathrm{aQmG14}$

[15.00]

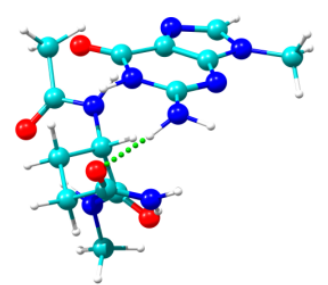

aQmG17

[16.71]

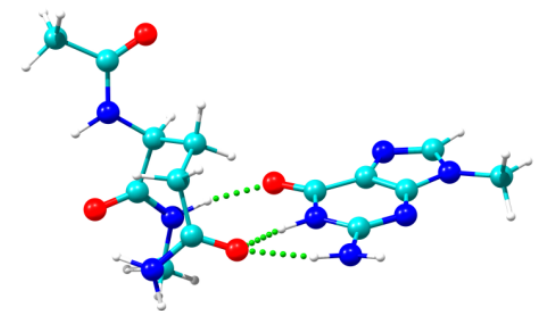

aQmG20

[17.63]

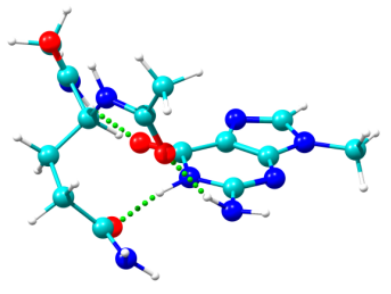

aQmG15

[15.35]

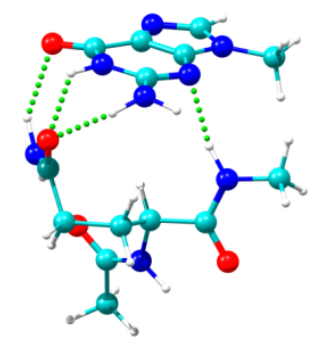

$\mathrm{aQmG18}$

[17.00] 
Figure S24. The first 20 calculated structures for $\mathrm{aRmG}$ at $\mathrm{M} 06-2 \mathrm{X} / 6-311++\mathrm{G}(\mathrm{d}, \mathrm{p})$ level together with their relative stability $\left(\mathrm{kJ} \mathrm{mol}^{-1}\right)$ in brackets. ZPE correction was applied to the energy values.

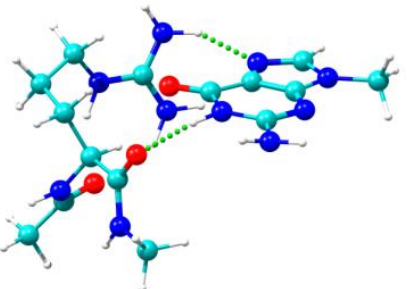

aRmG1

[0.00]

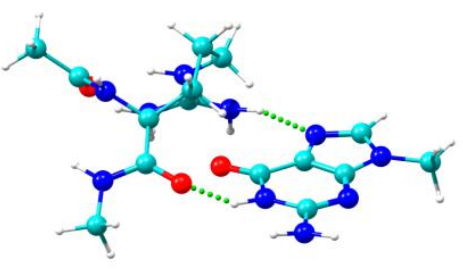

aRmG4

[7.21]

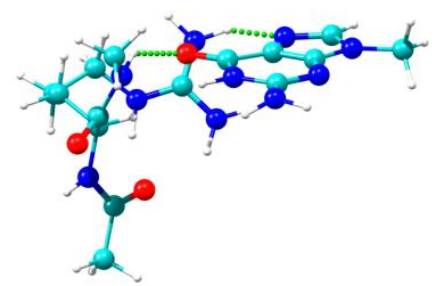

aRmG7

[10.59]

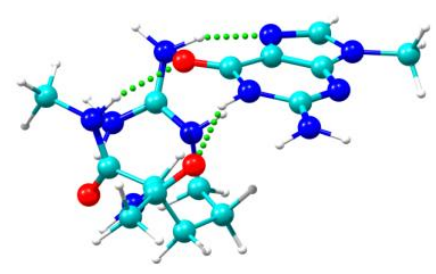

aRmG10

[12.42]

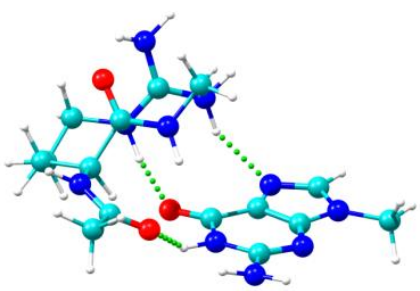

aRmG2

[4.64]

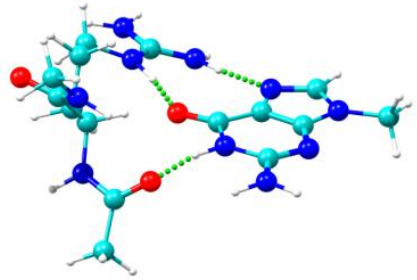

aRmG5

[8.36]

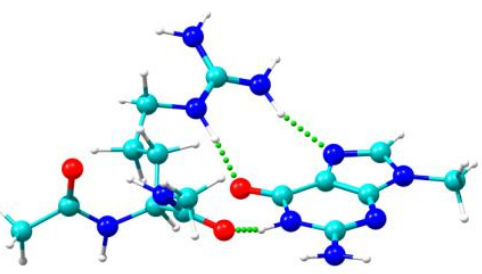

aRmG8

[10.82]

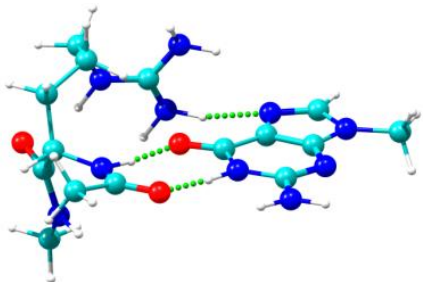

aRmG11

[13.41]

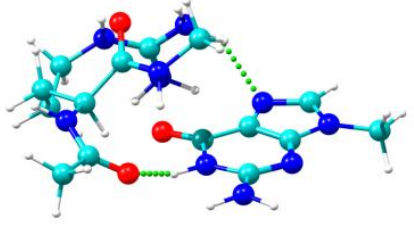

aRmG3

[7.05]

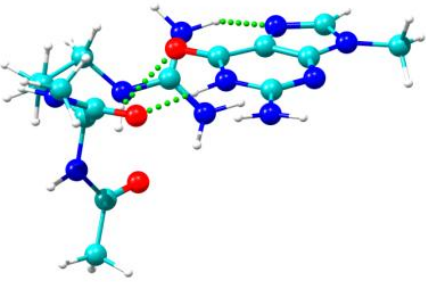

aRmG6

[8.87]

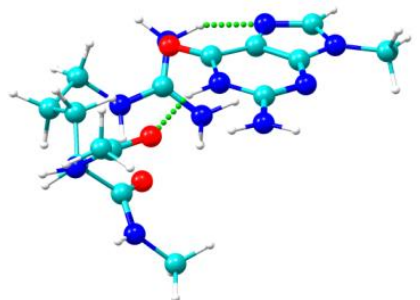

aRmG9

[11.68]

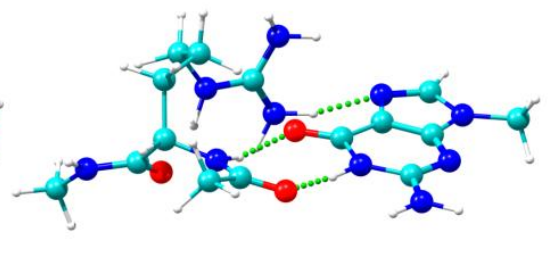

aRmG12

[15.02] 


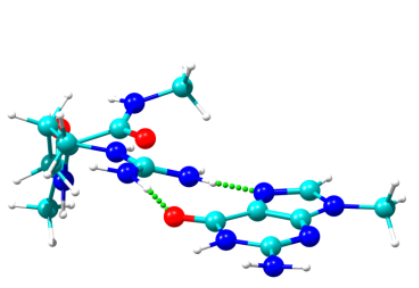

aRmG13

[17.93]

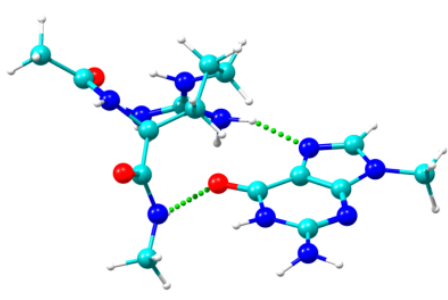

aRmG16

[18.45]

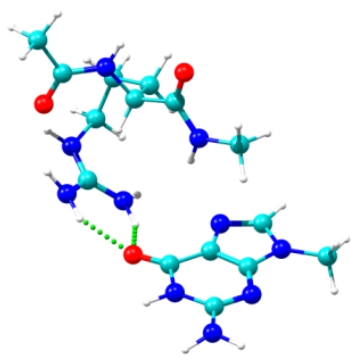

aRmG19

[19.96]

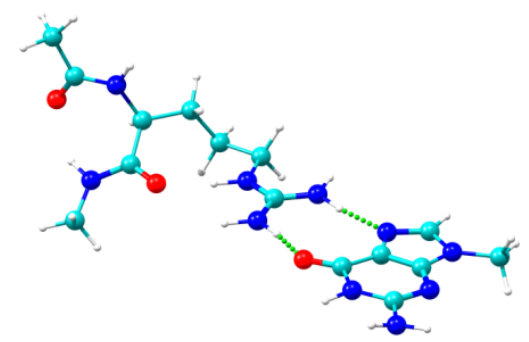

aRmG14

[17.93]

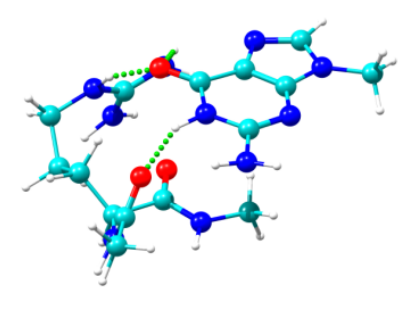

aRmG15

[17.95]

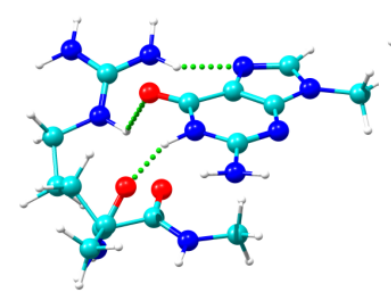

aRmG17

[18.91]

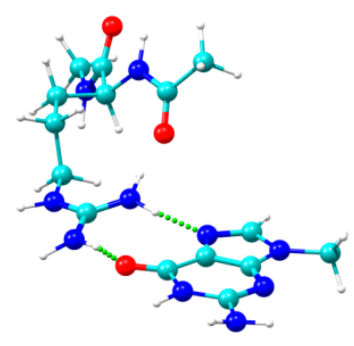

aRmG20

[20.42]

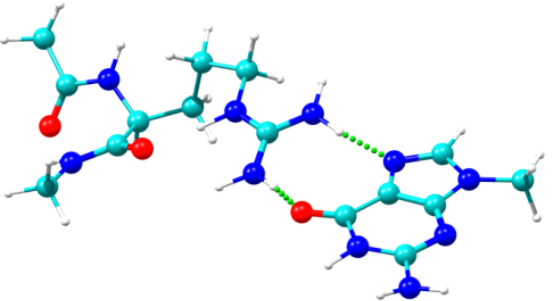

aRmG18

[19.89] 
Figure S25. The most stable structure for aAmC, aVmC and aImC calculated at the M06-2X/6-311++G(d,p) level. The hydrogen bonds and most relevant van der Waals interactions found are also marked. Three views of the NCI plots are presented for each structure.
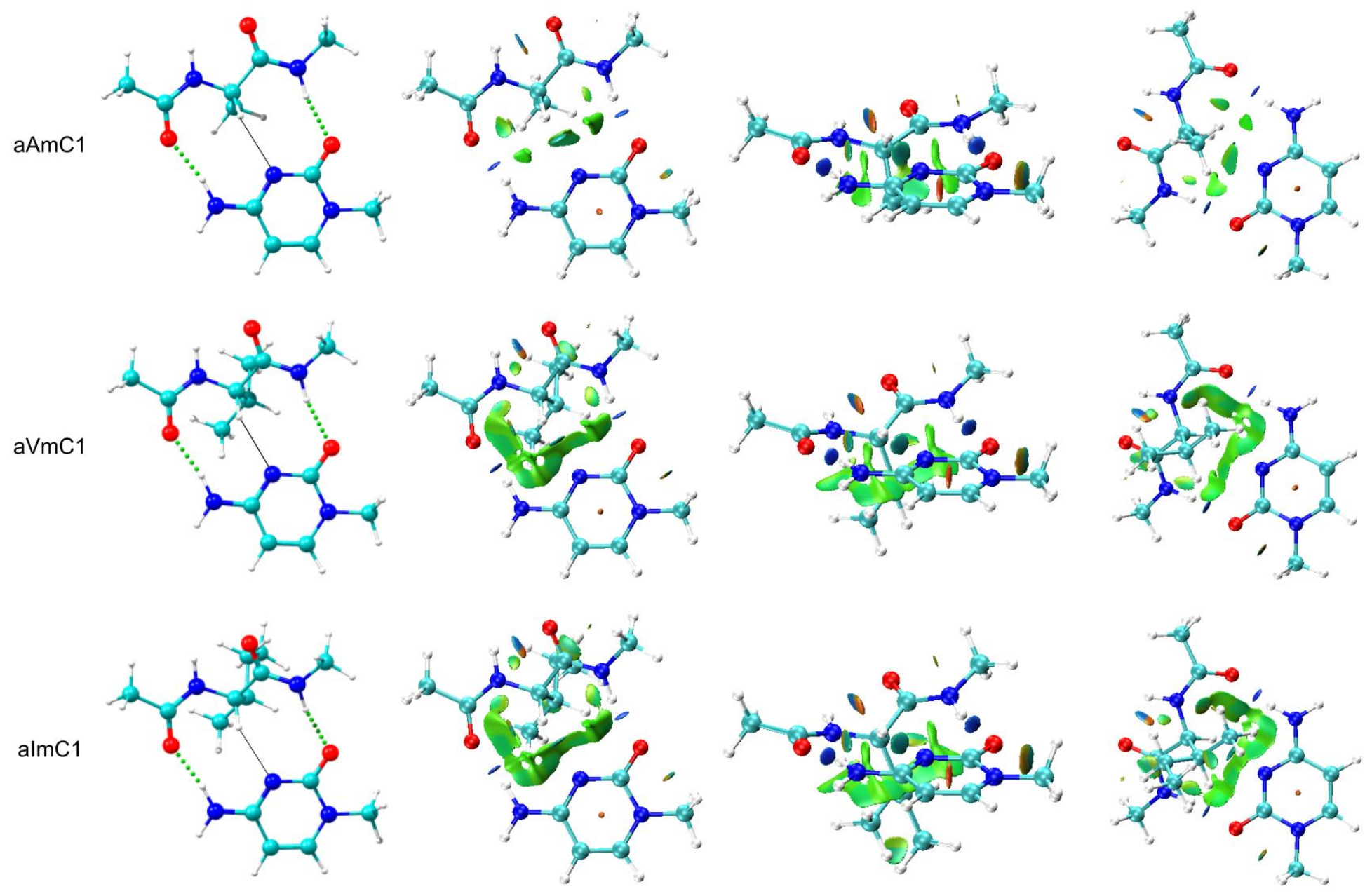
Figure S26. The most stable structure for aNmC, aQmC and aRmC calculated at the M06-2X/6-311++G(d,p) level. The hydrogen bonds and most relevant van der Waals interactions found are also marked. Three views of the NCI plots are presented for each structure.
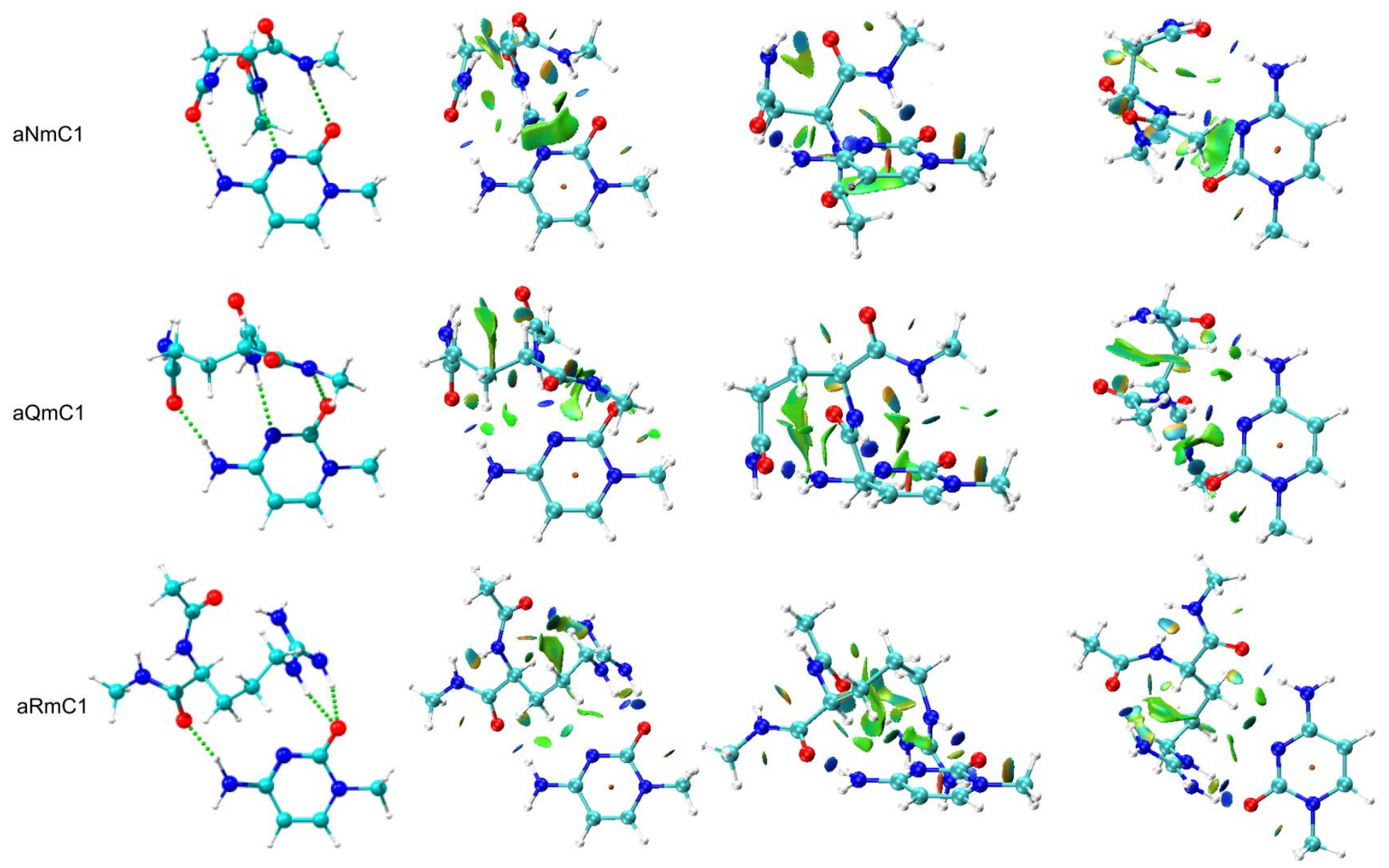
Figure S27. The most stable structure for aAmT, aVmT and aImT calculated at the M06-2X/6-311++G(d,p) level. The hydrogen bonds and most relevant van der Waals interactions found are also marked. Three views of the NCI plots are presented for each structure.
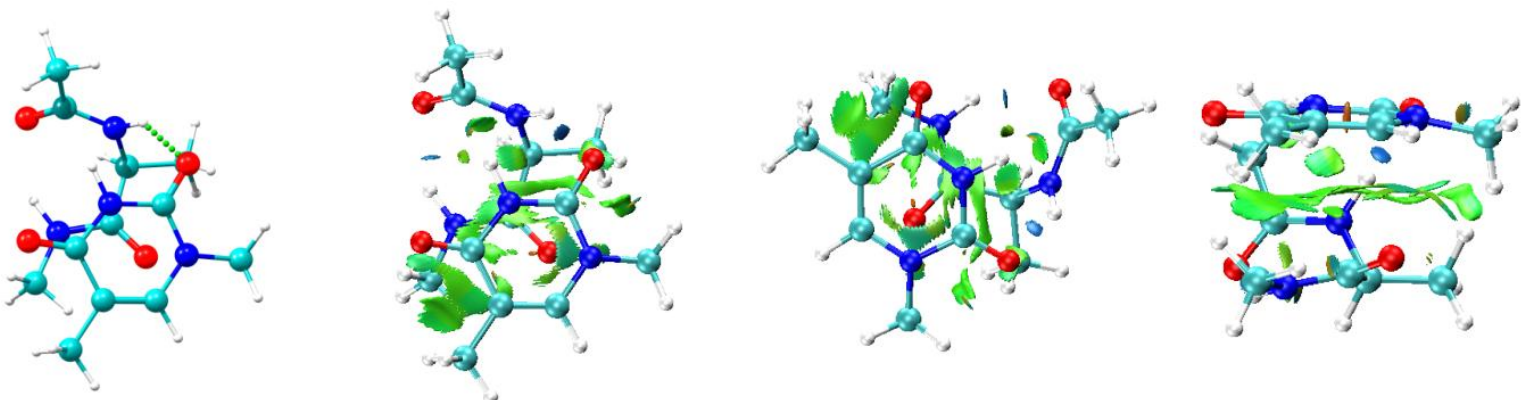

$\mathrm{aVmT1}$
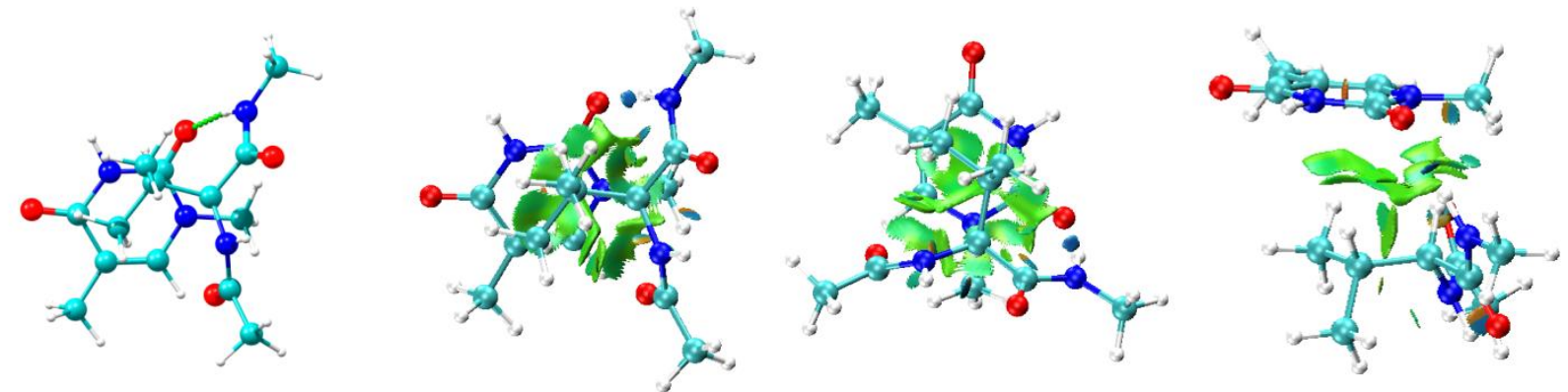

almT
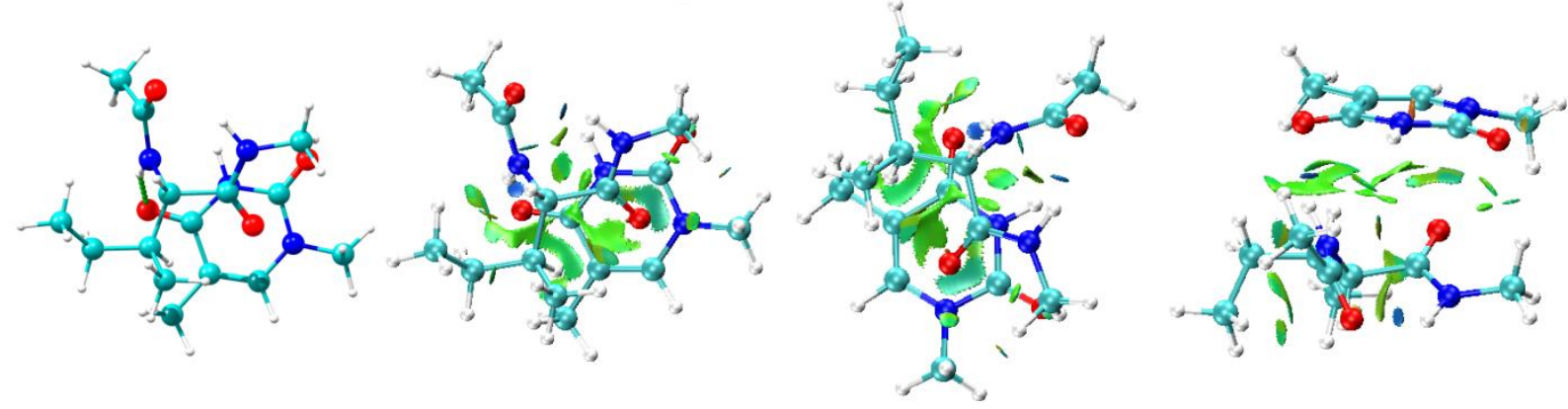
Figure S28. The most stable structure for aNmT, aQmT and aRmT calculated at the M06-2X/6-311++G(d,p) level. The hydrogen bonds and most relevant van der Waals interactions found are also marked. Three views of the NCI plots are presented for each structure.

aNmT1

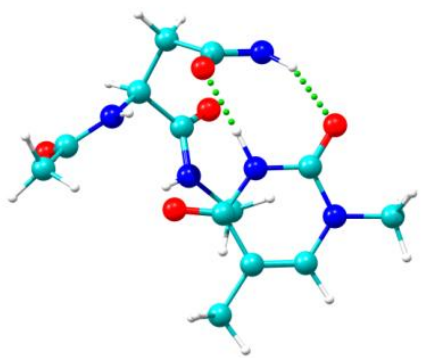

aQmT1
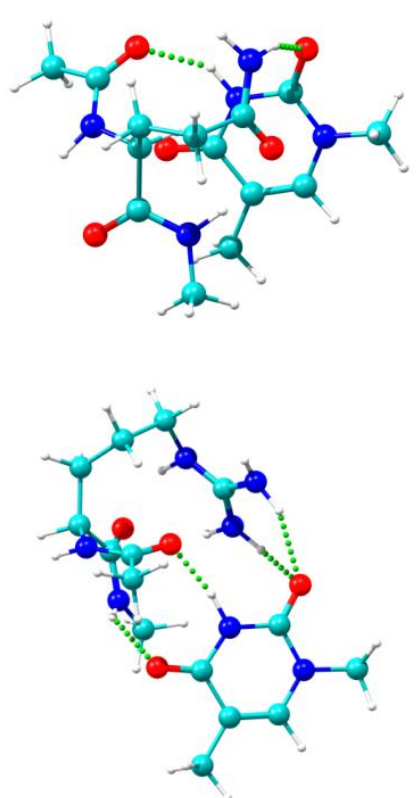
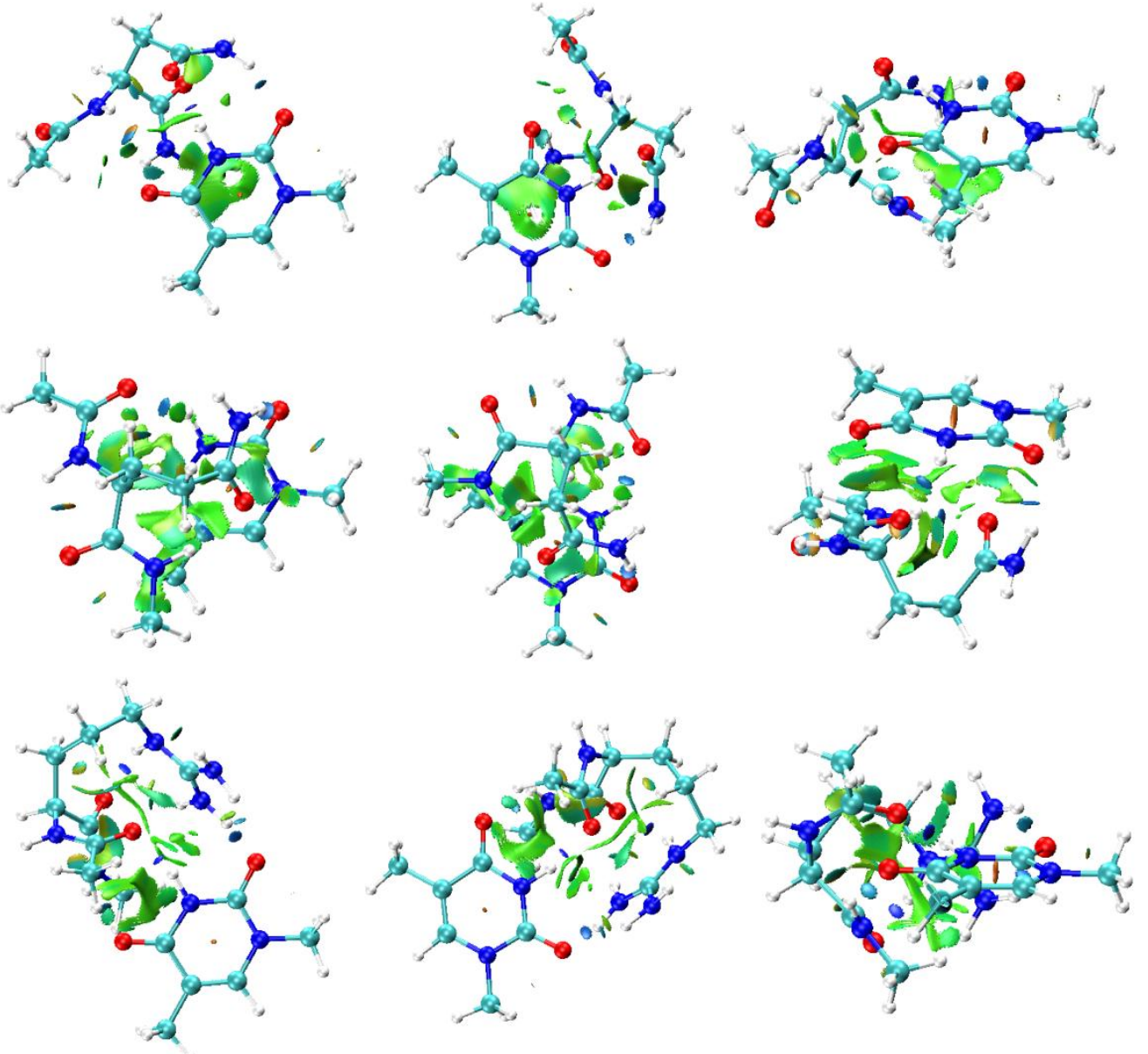
Figure S29. The most stable structure for aAmA, aVmA and aImA calculated at the M06-2X/6-311++G(d,p) level. The hydrogen bonds and most relevant van der Waals interactions found are also marked. Three views of the NCI plots are presented for each structure.

aAmA1

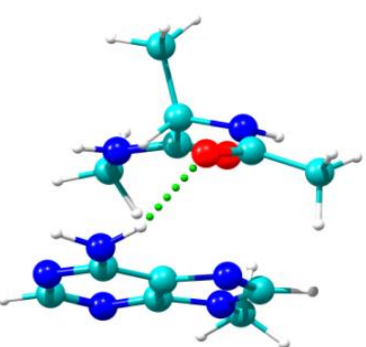

aVmA1

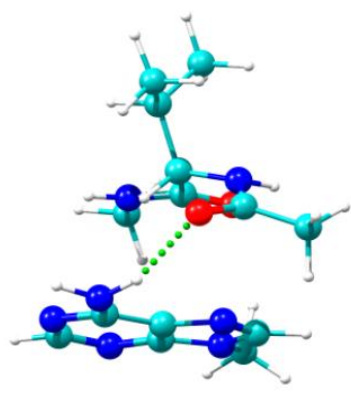

$\operatorname{almA1}$

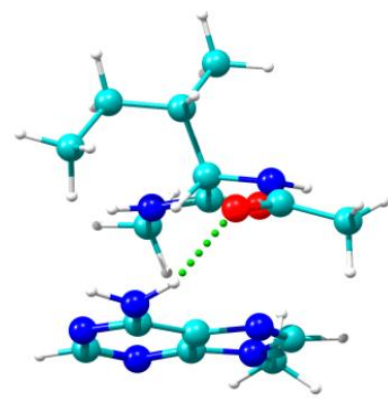

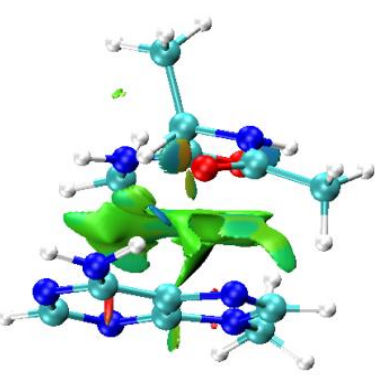
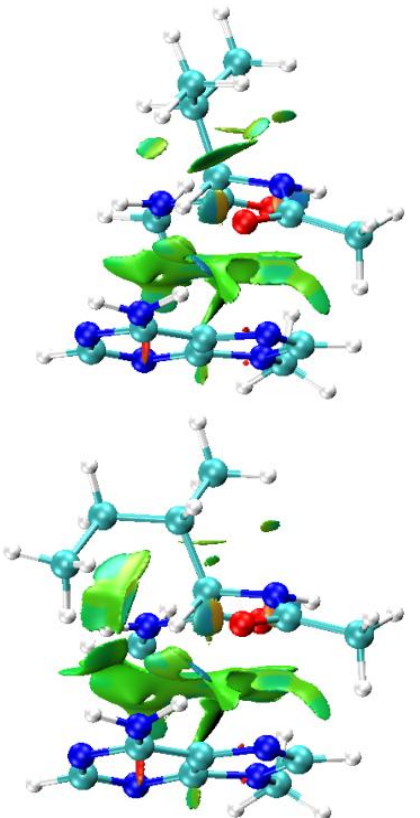
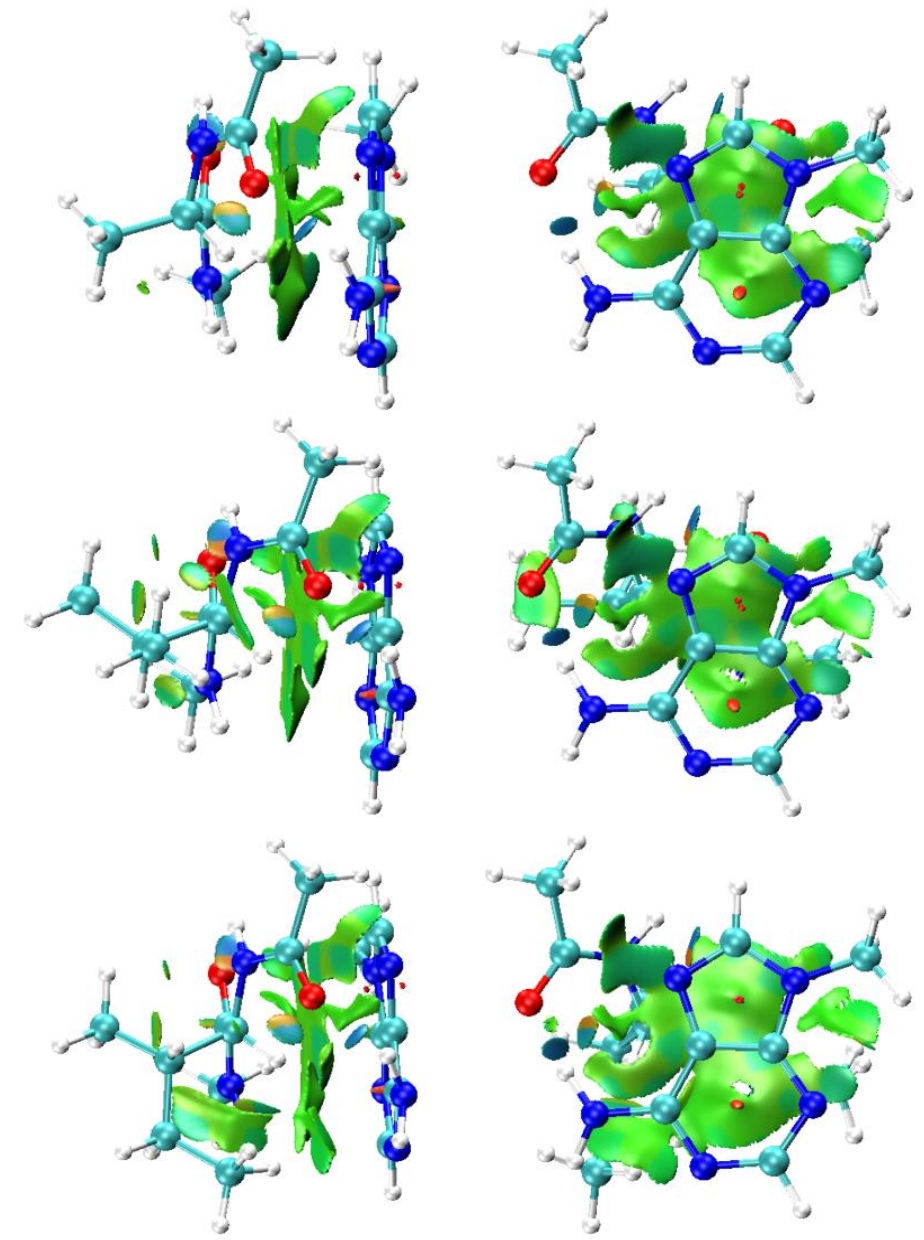
Figure S30. The most stable structure for aNmA, aQmA and aRmA calculated at the M06-2X/6-311++G(d,p) level. The hydrogen bonds and most relevant van der Waals interactions found are also marked. Three views of the NCI plots are presented for each structure.

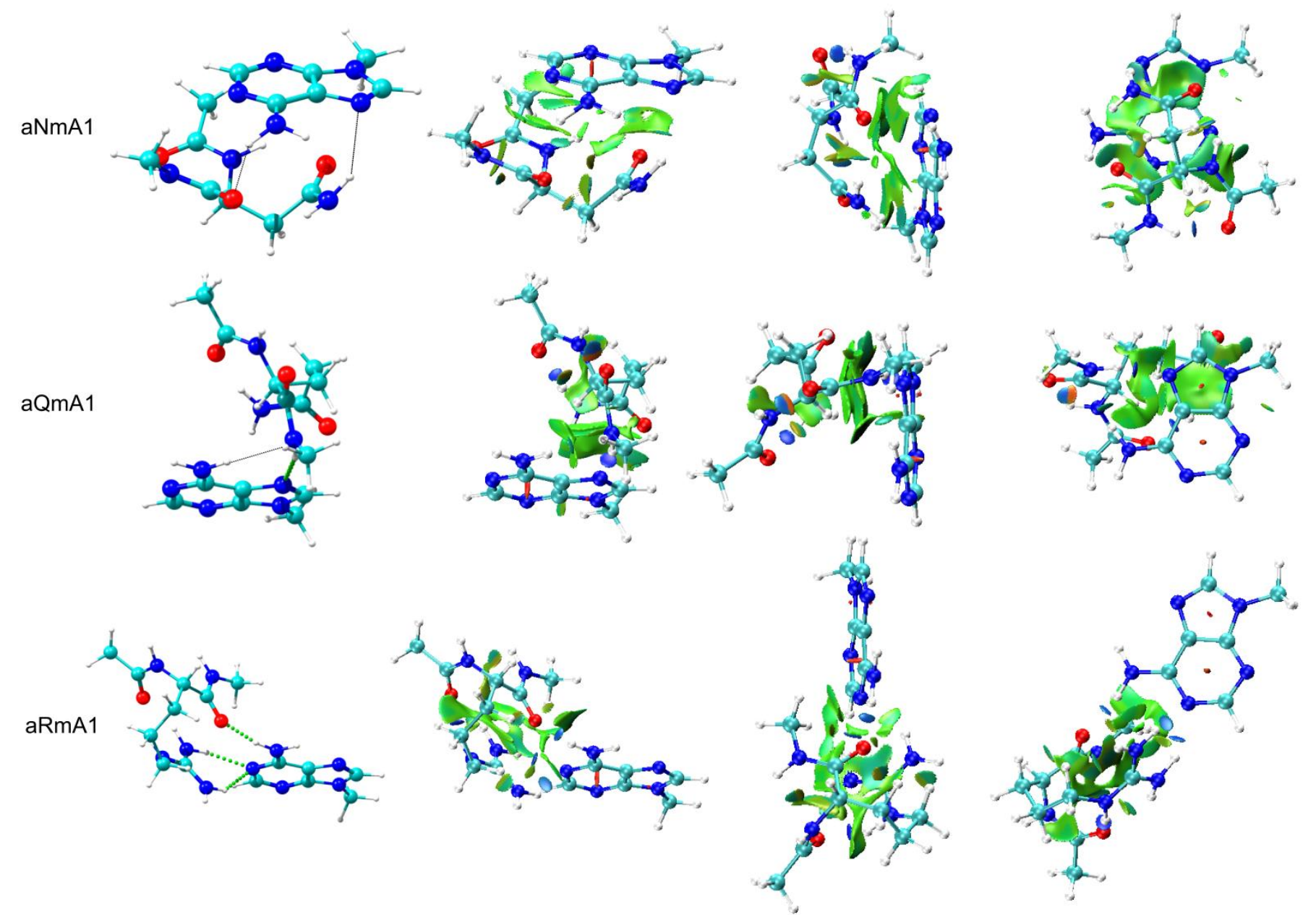


Figure S31. The most stable structure for aAmG, aVmG and aImG calculated at the M06-2X/6-311++G(d,p) level. The hydrogen bonds and most relevant van der Waals interactions found are also marked. Three views of the NCI plots are presented for each structure.

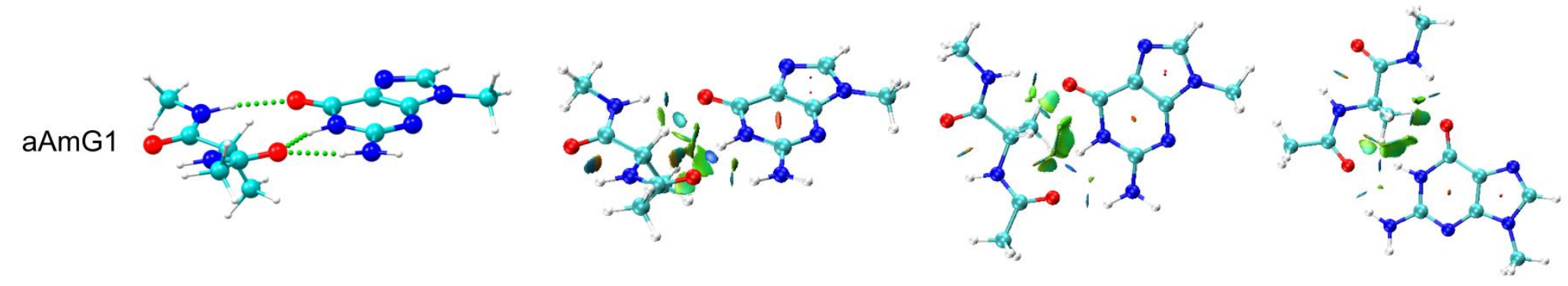

$\mathrm{aVmG1}$
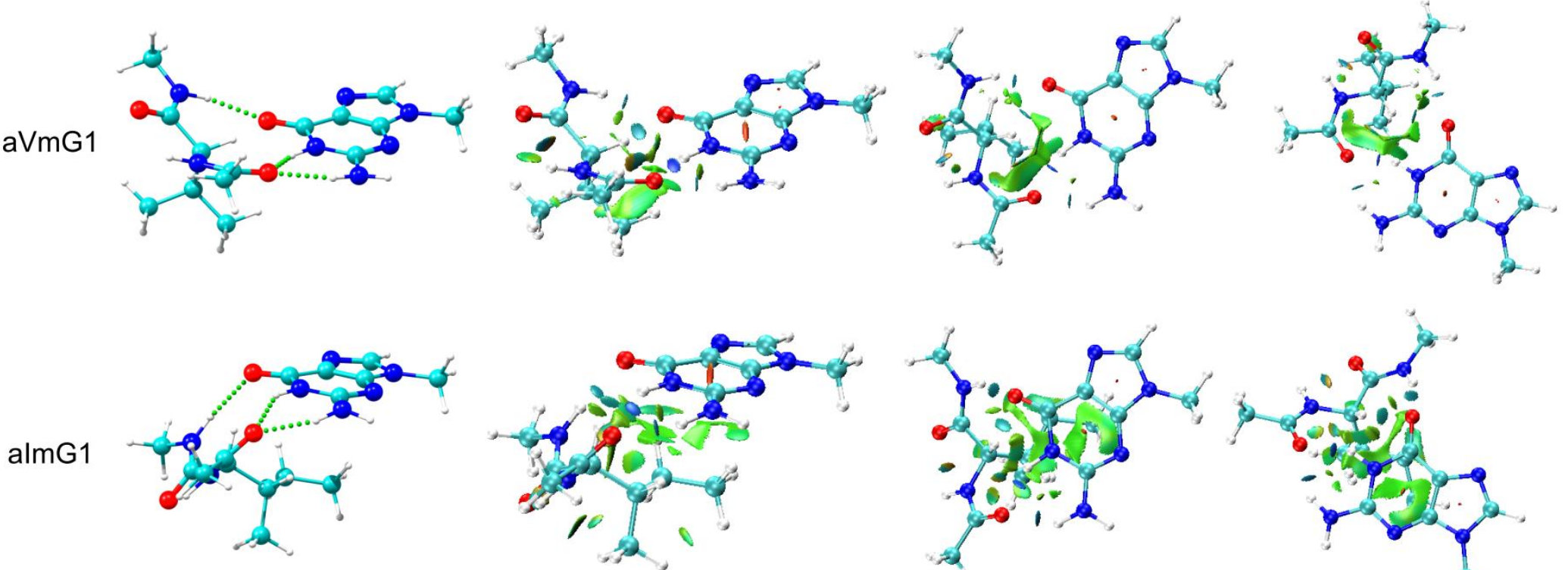
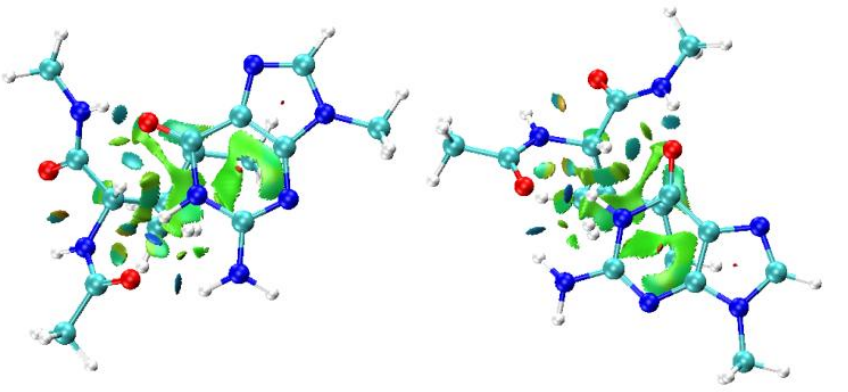
Figure S32. The most stable structure for aNmG, aQmG and aRmG calculated at the M06-2X/6-311++G(d,p) level. The hydrogen bonds and most relevant van der Waals interactions found are also marked. Three views of the NCI plots are presented for each structure.
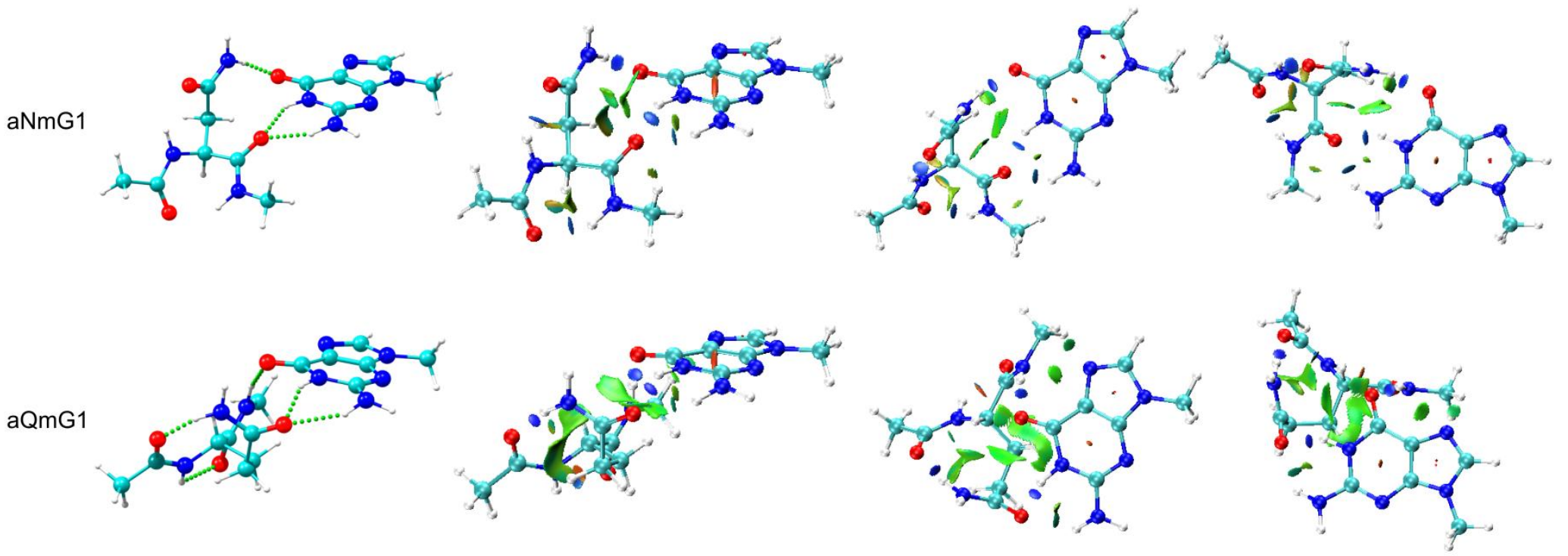

aRmG1
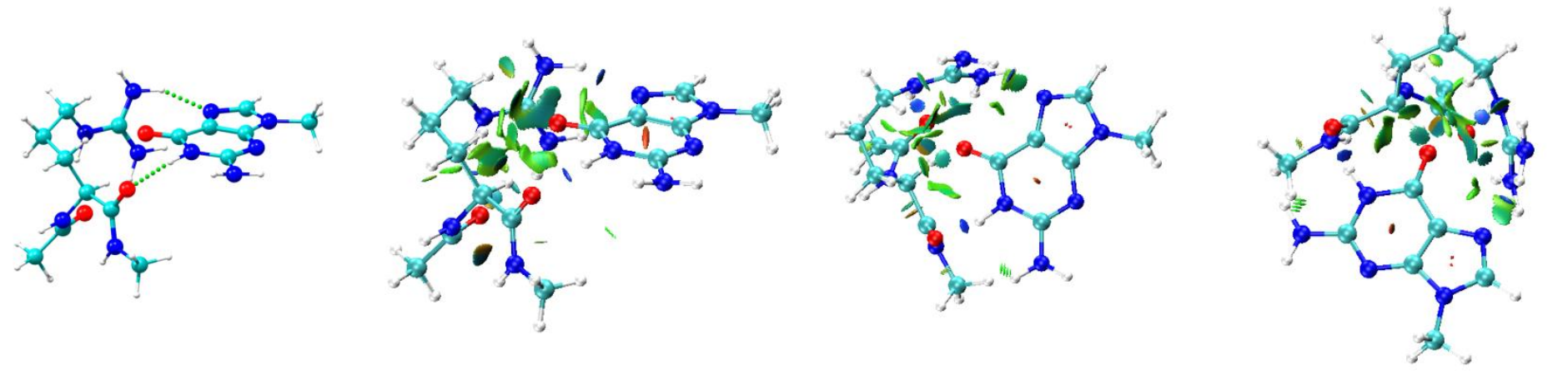
Figure S33. Correlation between the mean binding energy of amino acid-WC base pair complexes and the protein-base contact propensity ${ }^{15}$ for the most stable amino acid-WC base pair complexes investigated.

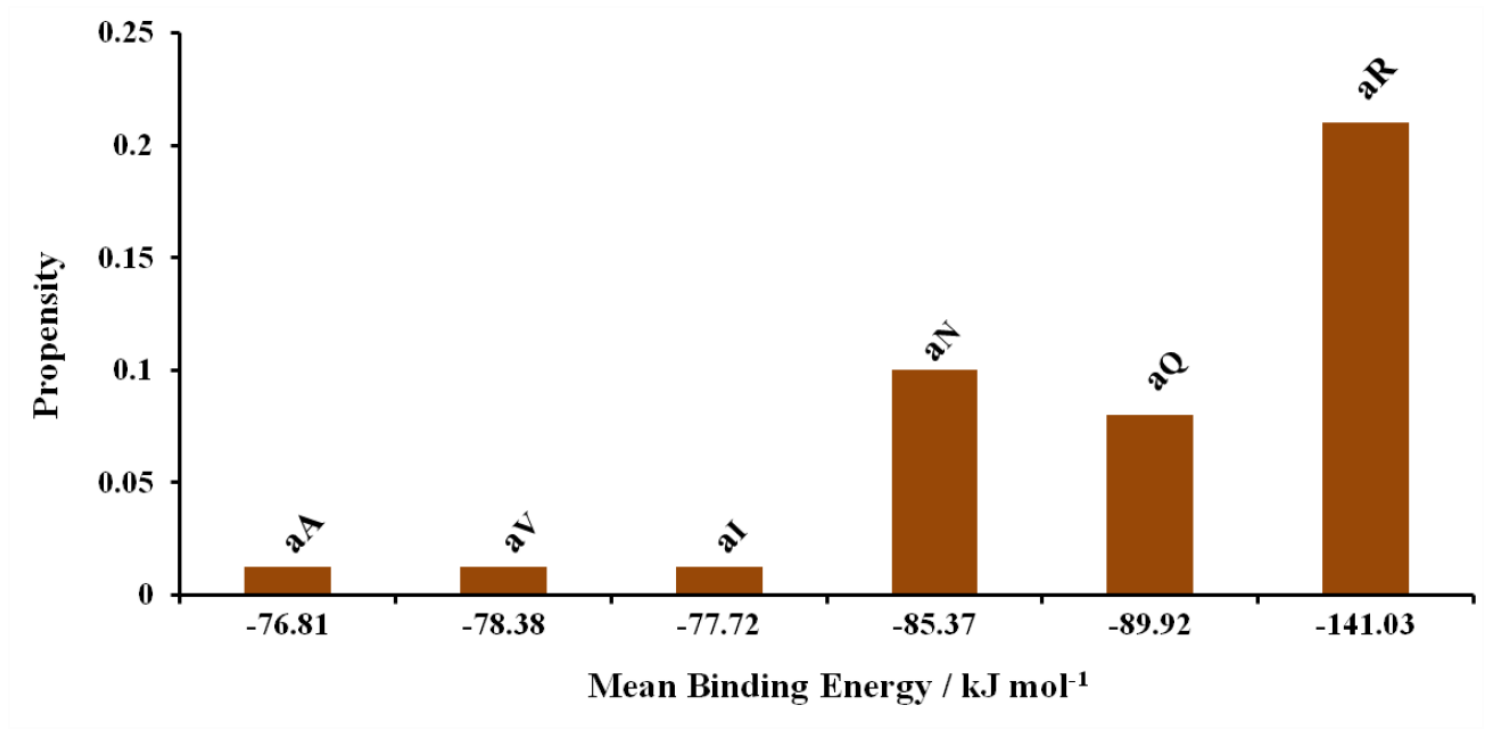


Table S01. Relative energies, ZPE and BSSE contributions, binding energies and the sum of electronic and thermal free energies at $310.15 \mathrm{~K}$ $(\Delta \mathrm{G})$ calculated at M06-2X/6-311++G(d,p) level for the three most stable structures for aAmC, aVmC, aImC, aNmC, aQmC and aRmC.

* Because the amino acid is not stable at M06-2X/6-311++G(d,p) level in the same conformation as that adopted in the cluster, the BSSE correction for these clusters was calculated with the amino acid in the most stable structure at M06-2X/6-311++G(d,p) level with the most similar conformation to which adopted in the cluster.

\begin{tabular}{|c|c|c|c|c|c|}
\hline Structure & $\Delta \mathrm{E}(\mathrm{kJ} / \mathrm{mol})$ & $\Delta \mathrm{E} \mathrm{ZPE}(\mathrm{kJ} / \mathrm{mol})$ & BSSE $(\mathrm{kJ} / \mathrm{mol})$ & Do $(\mathrm{kJ} / \mathrm{mol})$ & $\Delta \mathrm{G}_{310.15}(\mathrm{~kJ} / \mathrm{mol})$ \\
\hline $\mathrm{aAmC1}$ & 0.00 & 0.00 & 4.98 & -83.29 & 0.00 \\
\hline $\mathrm{aAmC} 2$ & 1.43 & 1.36 & 4.97 & -76.75 & 1.71 \\
\hline aAmC3 & 9.16 & 7.46 & 6.22 & -69.40 & 3.97 \\
\hline $\mathrm{aVmC1}$ & 0.00 & 0.00 & 5.78 & -85.07 & 0.00 \\
\hline $\mathrm{aVmC2}$ & 4.29 & 5.72 & 5.51 & -82.10 & 4.71 \\
\hline $\mathrm{aVmC} 3$ & 4.96 & 6.33 & 5.65 & -77.74 & 4.96 \\
\hline $\operatorname{aImC1} *$ & 0.00 & 0.00 & 6.12 & -83.76 & 0.00 \\
\hline $\mathrm{aImC2}$ & 2.20 & 3.08 & 5.85 & -79.31 & 2.38 \\
\hline $\operatorname{aImC3} *$ & 3.86 & 4.67 & 6.09 & -79.12 & 1.93 \\
\hline $\mathrm{aNmC1}$ & 0.00 & 0.00 & 7.43 & -72.05 & 0.00 \\
\hline $\mathrm{aNmC2}$ & 6.57 & 5.64 & 6.46 & -86.52 & 5.18 \\
\hline aNmC3 & 9.78 & 8.25 & 5.08 & -65.31 & 4.55 \\
\hline $\mathrm{aQmC1}$ & 0.00 & 0.00 & 7.32 & -88.42 & 0.00 \\
\hline $\mathrm{aQmC2}$ & 4.63 & 6.95 & 8.13 & -80.66 & 9.18 \\
\hline $\mathrm{aQmC3}$ & 5.48 & 8.13 & 5.26 & -70.34 & 9.18 \\
\hline $\mathrm{aRmC1}$ & 0.00 & 0.00 & 10.07 & -136.07 & 4.82 \\
\hline $\mathrm{aRmC2}$ & 3.33 & 0.20 & 8.86 & -127.29 & 0.00 \\
\hline aRmC3 & 4.62 & 4.22 & 10.78 & -131.15 & 9.45 \\
\hline
\end{tabular}


Table S02. Relative energies, ZPE and BSSE contributions, binding energies and the sum of electronic and thermal free energies at $310.15 \mathrm{~K}$ $(\Delta \mathrm{G})$ calculated at M06-2X/6-311++G(d,p) level for the three most stable structures for aAmT, aVmT, aImT, aNmT, aQmT and aRmT.

\begin{tabular}{|c|c|c|c|c|c|}
\hline Structure & $\Delta \mathrm{E}(\mathrm{kJ} / \mathrm{mol})$ & $\Delta \mathrm{E} \mathrm{ZPE}(\mathrm{kJ} / \mathrm{mol})$ & $\mathrm{BSSE}(\mathrm{kJ} / \mathrm{mol})$ & Do $(\mathrm{kJ} / \mathrm{mol})$ & $\Delta \mathrm{G}_{310.15}(\mathrm{~kJ} / \mathrm{mol})$ \\
\hline aAmT1 & 0.00 & 0.00 & 6.85 & -55.15 & 0.37 \\
\hline aAmT2 & 0.35 & 2.35 & 6.46 & -53.20 & 7.55 \\
\hline aAmT3 & 3.56 & 3.36 & 3.91 & -54.73 & 0.00 \\
\hline $\mathrm{aVmT1}$ & 2.40 & 0.00 & 6.13 & -57.88 & 0.00 \\
\hline $\mathrm{aVmT2}$ & 0.00 & 0.41 & 6.93 & -55.54 & 7.67 \\
\hline $\mathrm{aVmT3}$ & 4.78 & 2.72 & 6.44 & -57.32 & 4.67 \\
\hline $\operatorname{aImT1}$ & 0.00 & 0.00 & 6.81 & -57.47 & 3.90 \\
\hline $\mathrm{aImT2}$ & 2.36 & 0.51 & 6.50 & -58.91 & 0.00 \\
\hline aImT3 & 2.69 & 2.11 & 7.04 & -56.76 & 3.74 \\
\hline aNmT1 & 2.16 & 0.00 & 6.67 & -56.07 & 0.00 \\
\hline aNmT2 & 0.00 & 0.36 & 8.71 & -52.83 & 5.12 \\
\hline aNmT3 & 2.44 & 0.90 & 6.54 & -55.29 & 2.78 \\
\hline $\mathrm{aQmT1}$ & 0.00 & 0.00 & 8.71 & -67.52 & 1.89 \\
\hline $\mathrm{aQmT2}$ & 5.37 & 3.35 & 5.15 & -65.21 & 0.00 \\
\hline $\mathrm{aQmT3}$ & 2.36 & 3.65 & 9.59 & -60.48 & 12.89 \\
\hline aRmT1 & 0.00 & 0.00 & 6.43 & -112.34 & 1.60 \\
\hline aRmT2 & 1.57 & 0.96 & 6.57 & -111.23 & 0.00 \\
\hline $\mathrm{aRmT3}$ & 13.65 & 9.87 & 6.44 & -102.91 & 5.65 \\
\hline
\end{tabular}


Table S03. Relative energies, ZPE and BSSE contributions, binding energies and the sum of electronic and thermal free energies at $310.15 \mathrm{~K}$ $(\Delta \mathrm{G})$ calculated at M06-2X/6-311++G(d,p) level for the three most stable structures for aAmA, aVmA, aImA, aNmA, aQmA and aRmA.

\begin{tabular}{|c|c|c|c|c|c|}
\hline Structure & $\Delta \mathrm{E}(\mathrm{kJ} / \mathrm{mol})$ & $\Delta \mathrm{E} Z \mathrm{ZPE}(\mathrm{kJ} / \mathrm{mol})$ & BSSE $(\mathrm{kJ} / \mathrm{mol})$ & Do $(\mathrm{kJ} / \mathrm{mol})$ & $\Delta \mathrm{G}_{310.15}(\mathrm{~kJ} / \mathrm{mol})$ \\
\hline aAmA1 & 0.00 & 0.00 & 8.14 & -54.68 & 6.56 \\
\hline aAmA3 & 3.19 & 5.79 & 6.12 & -45.72 & 12.68 \\
\hline $\mathrm{aVmA} 2$ & 3.16 & 2.49 & 4.45 & -57.08 & 0.00 \\
\hline $\mathrm{aVmA} 3$ & 7.69 & 7.01 & 6.53 & -51.61 & 8.78 \\
\hline $\mathrm{aImA1}$ & 0.00 & 0.00 & 8.62 & -56.52 & 8.54 \\
\hline $\operatorname{aImA3*}$ & 5.85 & 4.30 & 5.62 & -53.58 & 4.99 \\
\hline aNmA1 & 0.00 & 0.00 & 8.63 & -53.07 & 0.00 \\
\hline aNmA2 & 6.19 & 6.71 & 6.89 & -48.10 & 4.94 \\
\hline aNmA3 & 5.04 & 7.32 & 8.89 & -44.66 & 9.07 \\
\hline $\mathrm{aQmA1}$ & 0.00 & 0.00 & 7.00 & -61.48 & 0.00 \\
\hline $\mathrm{aQmA2}$ & 8.21 & 7.28 & 6.49 & -54.71 & 5.05 \\
\hline aRmA3 & 7.60 & 6.80 & 4.48 & -76.93 & 6.60 \\
\hline
\end{tabular}


Table S04. Relative energies, ZPE and BSSE contributions, binding energies and the sum of electronic and thermal free energies at $310.15 \mathrm{~K}$ $(\Delta \mathrm{G})$ calculated at M06-2X/6-311++G(d,p) level for the three most stable structures for aAmG, aVmG, aImG, aNmG, aQmG and aRmG.

\begin{tabular}{|c|c|c|c|c|c|}
\hline Structure & $\Delta \mathrm{E}(\mathrm{kJ} / \mathrm{mol})$ & $\Delta \mathrm{E} \mathrm{ZPE}(\mathrm{kJ} / \mathrm{mol})$ & $\mathrm{BSSE}(\mathrm{kJ} / \mathrm{mol})$ & Do $(\mathrm{kJ} / \mathrm{mol})$ & $\Delta \mathrm{G}_{310.15}(\mathrm{~kJ} / \mathrm{mol})$ \\
\hline aAmG1 & 0.00 & 0.00 & 4.41 & -94.80 & 0.00 \\
\hline aAmG2 & 2.06 & 2.40 & 4.23 & -87.38 & 4.87 \\
\hline $\mathrm{aAmG} 3$ & 7.44 & 6.97 & 5.65 & -81.39 & 8.62 \\
\hline $\mathrm{aVmG1}$ & 0.00 & 0.00 & 4.87 & -91.86 & 1.78 \\
\hline $\mathrm{aVmG} 2$ & 0.51 & 0.01 & 4.91 & -91.81 & 0.00 \\
\hline $\mathrm{aVmG3}$ & 0.83 & 0.22 & 4.90 & -90.49 & 3.10 \\
\hline aImG1 & 0.00 & 0.00 & 7.01 & -91.73 & 4.97 \\
\hline aImG2 & 1.87 & 0.72 & 4.98 & -91.40 & 0.00 \\
\hline aImG3 & 0.86 & 0.77 & 5.29 & -94.79 & 0.85 \\
\hline aNmG1 & 0.00 & 0.00 & 4.47 & -83.04 & 0.00 \\
\hline $\mathrm{aNmG} 2$ & 1.75 & 0.93 & 4.86 & -80.89 & 4.73 \\
\hline aNmG3 & 5.97 & 6.41 & 7.33 & -73.77 & 16.26 \\
\hline $\mathrm{aQmG1}$ & 0.00 & 0.00 & 5.92 & -92.80 & 0.33 \\
\hline $\mathrm{aQmG} 2$ & 1.97 & 0.54 & 7.93 & -92.76 & 0.00 \\
\hline $\mathrm{aQmG3}$ & 3.10 & 1.68 & 7.77 & -115.52 & 7.61 \\
\hline aRmG1 & 0.00 & 0.00 & 7.94 & -151.55 & 0.00 \\
\hline aRmG2 & 5.01 & 4.64 & 8.04 & -161.30 & 4.68 \\
\hline aRmG3 & 4.15 & 7.05 & 9.40 & -130.84 & 11.28 \\
\hline
\end{tabular}


Table S05. Distances $(\AA)$ and angles $\left(^{\circ}\right)$ of the interactions (hydrogen bond, H bond, and van der Waals, vdW) calculated at the M06-2X/6$311++\mathrm{G}(\mathrm{d}, \mathrm{p})$ level for the second and third conformers of aAmC, aVmC and aImC.

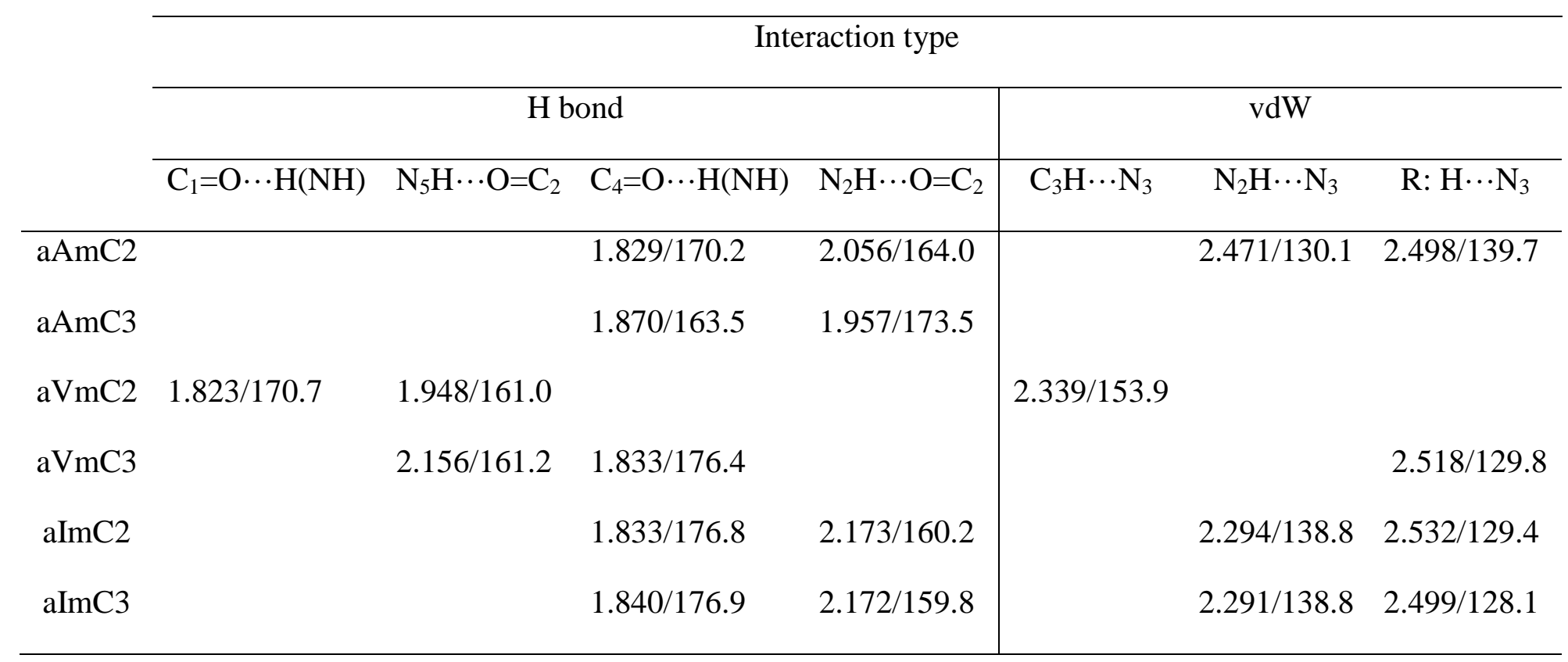


Table S06. Distances $(\AA)$ and angles $\left(^{\circ}\right)$ of the interactions (hydrogen bond, H bond, and van der Waals, vdW) calculated at the M06-2X/6$311++\mathrm{G}(\mathrm{d}, \mathrm{p})$ level for the second and third conformers of aNmC, aQmC and $\mathrm{aRmC}$.

\begin{tabular}{|c|c|c|c|c|c|c|c|}
\hline & \multicolumn{7}{|c|}{ Interaction type } \\
\hline & \multicolumn{7}{|c|}{$\mathrm{H}$ bond } \\
\hline & $\mathrm{C}_{1}=\mathrm{O} \cdots \mathrm{H}(\mathrm{NH})$ & $\mathrm{N}_{5} \mathrm{H} \cdots \mathrm{O}=\mathrm{C}_{2}$ & $\mathrm{C}_{4}=\mathrm{O} \cdots \mathrm{H}(\mathrm{NH})$ & $\mathrm{N}_{2} \mathrm{H} \cdots \mathrm{N}_{3}$ & $\mathrm{R}: \mathrm{C}=\mathrm{O} \cdots \mathrm{H}(\mathrm{NH})$ & $\mathrm{R}: \mathrm{H} \cdots \mathrm{O}=\mathrm{C}_{2}$ & $\mathrm{R}: \mathrm{H} \cdots \mathrm{N}_{3}$ \\
\hline aNmC2 & & $2.078 / 148.1$ & & $2.146 / 155.2$ & $1.843 / 174.3$ & & \\
\hline aNmC3 & $1.832 / 167.6$ & & & & $1.872 / 176.1$ & & \\
\hline $\mathrm{aQmC2}$ & & & & $2.013 / 172.0$ & $1.813 / 176.8$ & & \\
\hline $\mathrm{aQmC3}$ & & $1.844 / 160.4$ & & & $1.774 / 170.6$ & & \\
\hline $\mathrm{aRmC} 2$ & $2.050 / 172.8$ & & & & & $1.812 / 171.3$ & $1.795 / 172.5$ \\
\hline $\mathrm{aRmC} 3$ & & & $1.909 / 168.0$ & & & $1.771 / 168.9$ & $1.904 / 172.4$ \\
\hline
\end{tabular}

\begin{tabular}{|c|c|c|c|}
\hline & \multicolumn{3}{|c|}{ Interaction type } \\
\hline & \multicolumn{3}{|c|}{$\mathrm{vdW}$} \\
\hline & $\mathrm{N}_{5} \mathrm{H} \cdots \mathrm{C}=\mathrm{O}_{2}$ & $\mathrm{R}: \mathrm{H} \cdots \mathrm{O}=\mathrm{C}_{2}$ & $\mathrm{R}: \mathrm{H} \cdots \mathrm{N}_{3}$ \\
\hline $\mathrm{aNmC2}$ & & $2.337 / 134.5$ & \\
\hline $\mathrm{aNmC3}$ & & & $2.479 / 158.3$ \\
\hline $\mathrm{aQmC2}$ & $2.151 / 134.2$ & & \\
\hline \multicolumn{4}{|l|}{$\mathrm{aQmC3}$} \\
\hline \multicolumn{4}{|l|}{$\mathrm{aRmC2}$} \\
\hline $\mathrm{aRmC} 3$ & & & \\
\hline
\end{tabular}


Table S07. Distances $(\AA)$ and angles $\left(^{\circ}\right)$ of the interactions (hydrogen bond, H bond, and van der Waals, vdW) calculated at the M06-2X/6$311++\mathrm{G}(\mathrm{d}, \mathrm{p})$ level for the second and third conformers of aAmT, aVmT and aImT.

\begin{tabular}{|c|c|c|c|c|c|c|}
\hline & \multicolumn{6}{|c|}{ Interaction type } \\
\hline & \multicolumn{4}{|c|}{$\mathrm{H}$ bond } & \multicolumn{2}{|c|}{$\mathrm{vdW}$} \\
\hline & $\mathrm{N}_{2} \mathrm{H} \cdots \mathrm{O}=\mathrm{C}_{4}$ & $\mathrm{C}_{4}=\mathrm{O} \cdots \mathrm{HN}_{3}$ & $\mathrm{~N}_{5} \mathrm{H} \cdots \mathrm{O}=\mathrm{C}_{2}$ & $\mathrm{~N}_{5} \mathrm{H} \cdots \mathrm{O}=\mathrm{C}_{4}$ & $\mathrm{C}_{4}=\mathrm{O} \cdots \pi$ & $\mathrm{R}: \mathrm{H} \cdots \pi$ \\
\hline aAmT2 & $2.014 / 152.9$ & & & & 2.900/- & \\
\hline aAmT3 & $1.934 / 157.3$ & $1.820 / 171.9$ & & & & \\
\hline aVmT2 & $1.987 / 157.0$ & & & & 2.800/- & \\
\hline $\mathrm{aVmT3}$ & & & $1.978 / 175.0$ & & & $\sim 2.800 /-$ \\
\hline $\operatorname{aImT2}$ & & & & $2.010 / 159.0$ & & \\
\hline aImT3 & $1.982 / 157.1$ & & & & & \\
\hline
\end{tabular}


Table S08. Distances $(\AA)$ and angles $\left(^{\circ}\right)$ of the interactions (hydrogen bond, H bond, and van der Waals, vdW) calculated at the M06-2X/6$311++\mathrm{G}(\mathrm{d}, \mathrm{p})$ level for the second and third conformers of aNmT, aQmT and aRmT.

\begin{tabular}{|c|c|c|c|c|c|c|c|c|}
\hline & \multicolumn{8}{|c|}{ Interaction type } \\
\hline & \multicolumn{5}{|c|}{$\mathrm{H}$ bond } & \multicolumn{3}{|c|}{$\mathrm{vdW}$} \\
\hline & $\mathrm{N}_{5} \mathrm{H} \cdots \mathrm{O}=\mathrm{C}_{2}$ & $\mathrm{C}_{1}=\mathrm{O} \cdots \mathrm{HN}_{3}$ & $\mathrm{R}: \mathrm{O}(\mathrm{C}) \cdots \mathrm{HN}_{3}$ & $\mathrm{R}: \mathrm{H}(\mathrm{NH}) \cdots \mathrm{O}=\mathrm{C}_{2}$ & $\mathrm{R}: \mathrm{H}(\mathrm{NH}) \cdots \mathrm{O}=\mathrm{C}_{4}$ & $\mathrm{H}_{5} \mathrm{~N} \cdots \mathrm{O}=\mathrm{C}_{2}$ & $\mathrm{R}: \mathrm{H}(\mathrm{NH}) \cdots \mathrm{O}=\mathrm{C}_{4}$ & $\mathrm{R}: \mathrm{H}^{\cdots} \cdots \pi$ \\
\hline aNmT2 & & & & & & & & $\sim 2.500 /-$ \\
\hline aNmT3 & $2.200 / 140.2$ & & $1.821 / 167.7$ & & $2.021 / 155.9$ & & & \\
\hline $\mathrm{aQmT2}$ & $1.974 / 150.4$ & & $1.719 / 174.1$ & & $2.034 / 162.3$ & & & \\
\hline $\mathrm{aQmT3}$ & & & & $2.107 / 146.9$ & & & $2.122 / 133.1$ & $\sim 2.800 /-$ \\
\hline \multirow[t]{2}{*}{ aRmT2 } & & & & & $2.107 / 146.8$ & $2.123 / 133.6$ & & \\
\hline & & & & & $2.020 / 144.4$ & & & \\
\hline \multirow[t]{2}{*}{$\mathrm{aRmT3}$} & & $1.793 / 162.3$ & & & $2.053 / 146.6$ & $2.154 / 131.8$ & & \\
\hline & & & & & $1.968 / 150.5$ & & & \\
\hline
\end{tabular}


Table S09. Distances $(\AA)$ and angles $\left(^{\circ}\right)$ of the interactions (hydrogen bond, H bond, and van der Waals, vdW) calculated at the M06-2X/6$311++\mathrm{G}(\mathrm{d}, \mathrm{p})$ level for the second and third conformers of aAmA, aVmA and aImA.

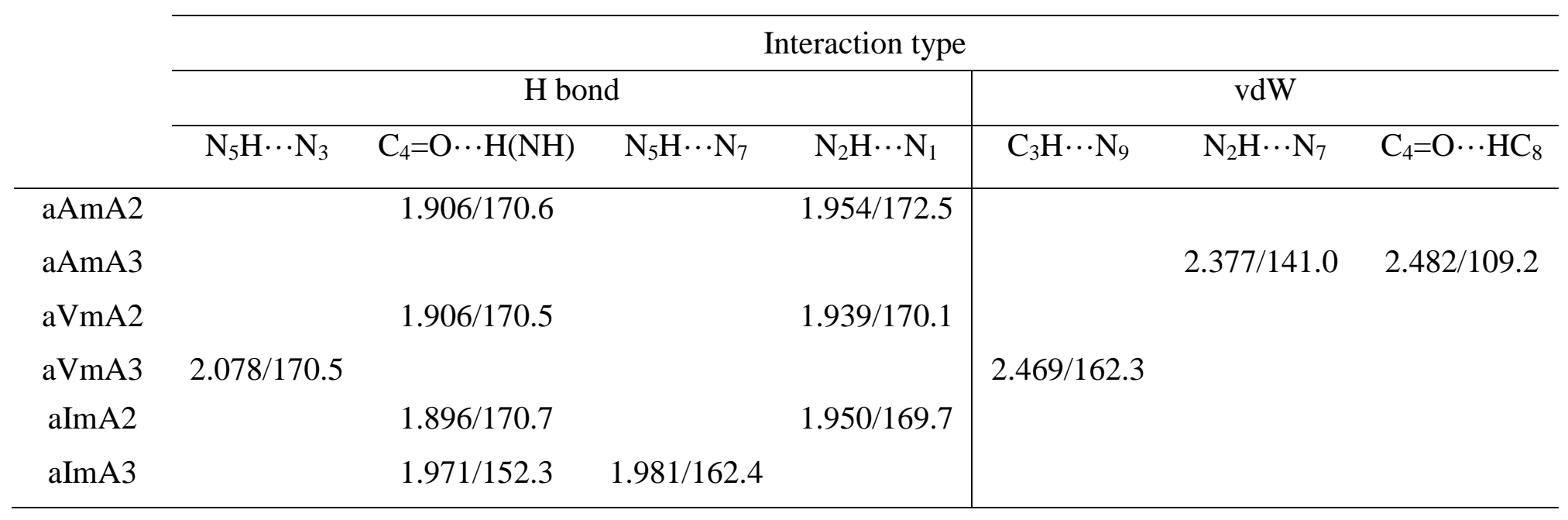


Table S10. Distances $(\AA)$ and angles $\left(^{\circ}\right)$ of the interactions (hydrogen bond, H bond, and van der Waals, vdW) calculated at the M06-2X/6$311++\mathrm{G}(\mathrm{d}, \mathrm{p})$ level for the second and third conformers of aNmA, aQmA and aRmA.

\begin{tabular}{|c|c|c|c|c|c|c|c|c|c|}
\hline & \multicolumn{9}{|c|}{ Interaction type } \\
\hline & \multicolumn{6}{|c|}{$\mathrm{H}$ bond } & \multicolumn{3}{|c|}{$\mathrm{vdW}$} \\
\hline & $\mathrm{C}_{1}=\mathrm{O} \cdots \mathrm{H}(\mathrm{NH})$ & $\mathrm{N}_{5} \mathrm{H} \cdots \mathrm{N}_{3}$ & $\mathrm{C}_{4}=\mathrm{O} \cdots \mathrm{H}(\mathrm{NH})$ & $\mathrm{N}_{5} \mathrm{H} \cdots \mathrm{H}(\mathrm{NH})$ & $\begin{array}{c}\mathrm{R}: \\
\mathrm{C}=\mathrm{O} \cdots \mathrm{H}(\mathrm{NH})\end{array}$ & $\mathrm{R}: \mathrm{H}(\mathrm{NH}) \cdots \mathrm{N}_{7}$ & $\mathrm{C}_{1}=\mathrm{O} \cdots \mathrm{H}(\mathrm{NH})$ & $\mathrm{C}_{3} \mathrm{H} \cdots \mathrm{N}\left(\mathrm{H}_{2}\right)$ & $\mathrm{R}: \mathrm{H} \cdots \mathrm{N}_{7}$ \\
\hline aNmA3 & & & & & & & $2.299 / 128.0$ & & \\
\hline $\mathrm{aQmA2}$ & & $1.955 / 170.9$ & & & & & & & $2.481 / 145.4$ \\
\hline aRmA2 & $2.094 / 144.7$ & & & & & $1.987 / 164.6$ & & $2.461 / 154.0$ & \\
\hline $\mathrm{aRmA3}$ & & & $2.016 / 150.5$ & & & $\begin{array}{l}2.079 / 151.1 \\
2.093 / 148.4\end{array}$ & & & \\
\hline
\end{tabular}


Table S11. Distances $(\AA)$ and angles $\left(^{\circ}\right)$ of the interactions (hydrogen bond, H bond, and van der Waals, vdW) calculated at the M06-2X/6$311++\mathrm{G}(\mathrm{d}, \mathrm{p})$ level for the second and third conformers of aAmG, aVmG and aImG.

\begin{tabular}{|c|c|c|c|c|c|c|c|c|}
\hline \multicolumn{9}{|c|}{ Interaction type } \\
\hline \multicolumn{8}{|c|}{$\mathrm{H}$ bond } & \multirow{2}{*}{$\frac{\mathrm{vdW}}{\mathrm{R}: \mathrm{H}(\mathrm{NH}) \cdots \mathrm{N}_{7}}$} \\
\hline $\mathrm{C}_{1}=\mathrm{O} \cdots \mathrm{H}(\mathrm{NH})$ & $\mathrm{C}_{1}=\mathrm{O} \cdots \mathrm{HN}_{1}$ & $\mathrm{~N}_{5} \mathrm{H} \cdots \mathrm{O}=\mathrm{C}_{6}$ & $\mathrm{~N}_{2} \mathrm{H} \cdots \mathrm{O}=\mathrm{C}_{6}$ & $\mathrm{C}_{4}=\mathrm{O} \cdots \mathrm{H}(\mathrm{NH})$ & $\mathrm{C}_{4}=\mathrm{O} \cdots \mathrm{HN}_{1}$ & $\mathrm{C}_{3} \mathrm{H} \cdots \mathrm{O}=\mathrm{C}_{6}$ & $\mathrm{R}: \mathrm{H}(\mathrm{NH}) \cdots \mathrm{O}=\mathrm{C}_{6}$ & \\
\hline \multirow{5}{*}{$2.067 / 144.3$} & & & $1.876 / 167.0$ & $2.075 / 145.7$ & $1.926 / 155.4$ & & $1.864 / 165.2$ & \\
\hline & & & $1.947 / 154.4$ & $2.038 / 146.2$ & $1.933 / 150.5$ & & & \\
\hline & $1.879 / 156.1$ & $1.980 / 163.7$ & & & & $2.307 / 143.9$ & & \\
\hline & & & $1.861 / 167.4$ & $2.059 / 146.0$ & $1.927 / 154.7$ & & & $2.167 / 169.5$ \\
\hline & & & $1.862 / 167.4$ & $2.073 / 145.8$ & $1.923 / 155.0$ & & & $2.077 / 145.9$ \\
\hline $2.058 / 145.1$ & $1.893 / 153.9$ & $1.928 / 166.4$ & & & & & & $2.150 / 144.4$ \\
\hline
\end{tabular}


Table S12. Distances $(\AA)$ and angles $\left(^{\circ}\right)$ of the interactions (hydrogen bond, H bond, and van der Waals, vdW) calculated at the M06-2X/6$311++\mathrm{G}(\mathrm{d}, \mathrm{p})$ level for the second and third conformers of $\mathrm{aNmG}, \mathrm{aQmG}$ and $\mathrm{aRmG}$.

\begin{tabular}{|c|c|c|c|c|c|c|c|c|}
\hline & \multicolumn{8}{|c|}{ Interaction type } \\
\hline & \multicolumn{5}{|c|}{$\mathrm{H}$ bond } & \multicolumn{3}{|c|}{$\mathrm{vdW}$} \\
\hline & $\mathrm{C}_{1}=\mathrm{O} \cdots \mathrm{H}(\mathrm{NH})$ & $\mathrm{C}_{1}=\mathrm{O} \cdots \mathrm{HN}_{1}$ & $\mathrm{~N}_{5} \mathrm{H} \cdots \mathrm{O}=\mathrm{C}_{6}$ & $\mathrm{~N}_{2} \mathrm{H} \cdots \mathrm{O}=\mathrm{C}_{6}$ & $\mathrm{R}: \mathrm{H} \cdots \mathrm{O}=\mathrm{C}_{6}$ & $\mathrm{C}_{3} \mathrm{H} \cdots \mathrm{O}=\mathrm{C}_{6}$ & $\mathrm{R}: \mathrm{H}(\mathrm{NH}) \cdots \mathrm{N}_{7}$ & $\mathrm{R}: \mathrm{O}(\mathrm{C}) \cdots \pi$ \\
\hline $\mathrm{aNmG} 2$ & $2.040 / 144.8$ & $1.914 / 153.1$ & & & & & & \\
\hline aNmG3 & & & & $1.921 / 162.9$ & & & & \\
\hline $\mathrm{aQmG} 2$ & $2.064 / 146.4$ & $1.956 / 148.0$ & $1.865 / 170.2$ & & & & $2.230 / 163.7$ & 23.100/ - \\
\hline $\mathrm{aQmG3}$ & $2.135 / 144.7$ & $1.925 / 147.7$ & $1.908 / 149.7$ & & & & & $\sim 2.900 /-$ \\
\hline $\mathrm{aRmG} 2$ & & $1.917 / 142.0$ & & & $1.774 / 153.4$ & $2.341 / 143.1$ & & \\
\hline aRmG3 & & $1.931 / 153.5$ & & & & & $2.329 / 243.5$ & \\
\hline
\end{tabular}

